\title{
Noncommutative algebraic geometry
}

\section{Olav A. Laudal}

\begin{abstract}
The need for a noncommutative algebraic geometry is apparent in classical invariant and moduli theory. It is, in general, impossible to find commuting parameters parametrizing all orbits of a Lie group acting on a scheme. When one orbit is contained in the closure of another, the orbit space cannot, in a natural way, be given a scheme structure.

In this paper we shall show that one may overcome these difficulties by introducing a noncommutative algebraic geometry, where affine "schemes" are modeled on associative algebras. The points of such an affine scheme are the simple modules of the algebra, and the local structure of the scheme at a finite family of points, is expressed in terms of a noncommutative deformation theory proposed by the author in [10].

More generally, the geometry in the theory is represented by a swarm, i.e. a diagram (finite or infinite) of objects (and if one wants, arrows) in a given $k$-linear Abelian category ( $k$ a field), satisfying some reasonable conditions. The noncommutative deformation theory refered to above, permits the construction of a presheaf of associative $k$-algebras, locally parametrizing the diagram. It is shown that this theory, in a natural way, generalizes the classical scheme theory. Moreover it provides a promising framework for treating problems of invariant theory and moduli problems. In particular it is shown that many moduli spaces in classical algebraic geometry are commutativizations of noncommutative schemes containing additional information.
\end{abstract}

2000 Mathematics Subject Classification: 14A22, 16E, 16D90, 16G, 13D.

Keywords: Modules, noncommutative deformations of modules, Massey products, swarm of modules, algebra of observables, noncommutative schemes, invariant theory, moduli spaces. 


\section{Introduction}

In this paper I shall first, sketch, or recall from [10] a noncommutative deformation theory for modules on some $k$-algebra $A, k$ a field. Then, in $\S 3-4$ I shall apply it to construct a noncommutative algebraic geometry. The final paragraphs of the paper are concerned with the application to invariant and moduli theory, and to some examples.

The basic idea of noncommutative deformation theory is very simple. Let $\underline{a}_{r}$ denote the category of $r$-pointed not necessarily commutative $k$ algebras $R$. The objects are the diagrams of $k$-algebras,

$$
k^{r} \stackrel{\iota}{\rightarrow} R \stackrel{\rho}{\rightarrow} k^{r}
$$

such that the composition of $\iota$ and $\rho$ is the identity. Any such r-pointed $k$ algebra $R$ is isomorphic to a $k$-algebra of $r \times r$-matrices $\left(R_{i, j}\right)$. The radical of $R$ is the bilateral ideal $\operatorname{Rad}(R):=\operatorname{ker} \rho$. The dual $k$-vector space of $\operatorname{Rad}(R) / \operatorname{Rad}(R)^{2}$ is called the tangent space of $R$.

For $r=1$, there is an obvious inclusion of categories

$$
\underline{l} \subseteq \underline{a}_{1}
$$

where $\underline{l}$, as usual, denotes the category of commutative local artinian $k$ algebras with residue field $k$.

Fix a not necessarily commutative $k$-algebra $A$ and consider a right $A$ module $M$. The classical deformation functor

$$
\operatorname{Def}_{M}: \underline{l} \rightarrow \underline{\text { Sets }}
$$

is then defined. Assuming $\operatorname{Ext}_{A}^{i}(M, M)$ has finite $k$-dimension for $i=1,2$, it is well known, see [17] or [8], that $\operatorname{Def}_{M}$ has a noetherian prorepresenting hull $H$, the formal moduli of $M$. Moreover, the tangent space of $H$ is isomorphic to $\operatorname{Ext}_{A}^{1}(M, M)$, and $H$ can be computed in terms of $\operatorname{Ext}_{A}^{i}(M, M), i=1,2$ and their matric Massey products, see [7], [8].

In the general case, consider a finite family $\mathcal{V}=\left\{V_{i}\right\}_{i=1}^{r}$ of $A$-modules and define a deformation functor,

$$
\operatorname{Def}_{\mathcal{V}}: \underline{a}_{r} \rightarrow \underline{\text { Sets }}
$$

generalizing the functor $\operatorname{Def}_{M}$ above. Given an object $\rho: R=\left(R_{i, j}\right) \rightarrow k^{r}$ of $\underline{a}_{r}$, consider the $k$-vector space and $R$-left module $\left(R_{i, j} \otimes_{k} V_{j}\right) . \rho$ defines a $k$-linear and left $R$-linear map,

$$
\rho(R):\left(R_{i, j} \otimes_{k} V_{j}\right) \rightarrow \oplus_{i=1}^{r} V_{i},
$$

inducing a homomorphism of $R$-endomorphism rings,

$$
\tilde{\rho}(R):\left(R_{i, j} \otimes_{k} \operatorname{Hom}_{k}\left(V_{i}, V_{j}\right)\right) \rightarrow \oplus_{i=1}^{r} \operatorname{End}_{k}\left(V_{i}\right) .
$$


The right $A$-module structure on the $V_{i}$ 's is defined by a homomorphism of $k$-algebras, $\eta_{0}: A \rightarrow \oplus_{i=1}^{r} \operatorname{End}_{k}\left(V_{i}\right)$. Let $\operatorname{Def}_{\mathcal{V}}(R) \in \underline{\text { Sets }}$ be the set of isoclasses of homomorphisms of $k$-algebras,

$$
\eta^{\prime}: A \rightarrow\left(R_{i, j} \otimes_{k} \operatorname{Hom}_{k}\left(V_{i}, V_{j}\right)\right)
$$

such that, $\tilde{\rho}(R) \circ \eta^{\prime}=\eta_{0}$, where the equivalence relation is defined by inner automorphisms in the $k$-algebra $\left(R_{i, j} \otimes_{k} \operatorname{Hom}_{k}\left(V_{i}, V_{j}\right)\right)$. Assume that for all $0 \leq i, j \leq r$ we have

$$
\operatorname{dim}_{k} \operatorname{Ext}_{A}^{1}\left(V_{i}, V_{j}\right)<\infty .
$$

Then it is easy to see that $\operatorname{Def}_{\mathcal{V}}$ has the same properties as the ordinary deformation functor:

Theorem 2.3 The functor Def $\mathcal{V}$ has a prorepresentable hull, i.e. an object $H(\mathcal{V})$ of the procategory $\underline{\hat{a}}_{r}$, together with a formal versal family,

$$
\tilde{V}=\left(H_{i, j} \otimes V_{j}\right) \in \lim _{n \geq 1} \operatorname{Def}_{\mathcal{V}}\left(H / \mathfrak{m}^{n}\right)
$$

such that the corresponding morphism of functors on $\underline{a}_{r}$,

$$
\rho: \operatorname{Mor}(H,-) \rightarrow \operatorname{Def}_{\mathcal{V}}
$$

is smooth, and an isomorphism on the tangent level. Moreover, $H$ is uniquely determined by a set of matric Massey products of the form

$$
\operatorname{Ext}^{1}\left(V_{i}, V_{j_{1}}\right) \otimes \cdots \otimes \operatorname{Ext}^{1}\left(V_{j_{n-1}}, V_{j}\right) \cdots \rightarrow \operatorname{Ext}^{2}\left(V_{i}, V_{j}\right) .
$$

Finite families of $A$-modules, $\mathcal{V}$ satisfying,

$$
\operatorname{dim}_{k} \operatorname{Ext}_{A}^{1}\left(V_{i}, V_{j}\right)<\infty,
$$

and for which there exists a natural right action of $A$ on $\tilde{V}$ will be called swarms, see (2.4). Any swarm defines a homomorphism of $k$-algebras,

$$
\eta: A \longrightarrow O(\mathcal{V}):=\operatorname{End}_{H}(\tilde{V})=\left(H_{i, j} \otimes \operatorname{Hom}_{k}\left(V_{i}, V_{j}\right)\right),
$$

and the $k$-algebra $O(\mathcal{V})$ acts on the family of $A$-modules $\mathcal{V}=\left\{V_{i}\right\}$, extending the action of $A$. If $\operatorname{dim}_{k} V_{i}<\infty$, for all $i=1, \ldots, r$, the operation of associating $(O(\mathcal{V}), \mathcal{V})$ to $(A, \mathcal{V})$ turns out to be a closure operation.

An important result, needed for the construction of the structure sheaf in noncommutative algebraic geometry, is the following,

Theorem 2.6 (A generalized Burnside theorem) Let $A$ be a finite dimensional $k$-algebra, $k$ an algebraically closed field. Consider the family $\mathcal{V}=\left\{V_{i}\right\}_{i=1}^{r}$ of simple A-modules, then

$$
A \simeq O(\mathcal{V})=\left(H_{i, j} \otimes \operatorname{Hom}_{k}\left(V_{i}, V_{j}\right)\right) .
$$


Based on this notion of noncommutative deformations, we propose a general definition of an affine noncommutative prescheme, and scheme, generalizing the classical notion of an affine algebraic scheme in the commutative case.

The construction is dependent upon the choice of a reasonable abelian category of objects $\underline{C}$, the universe. Since the process of generalizing will be clear, I shall assume that we are given an algebraically closed field $k$, a $k$-algebra $A$, and that we pick as our universe $\underline{C}$, the category $A$-mod of right $A$-modules.

As a model we shall take the classical construction of the structure sheaf $O_{X}$, of the scheme $X:=\operatorname{Spec}(A)$, when $A$ is a commutative finite type $k$-algebra. A point of $X$ is a prime ideal $\mathfrak{p}$ of $A$, or rather the right (or left) $A$-module $A / \mathfrak{p}$. A closed point $x \in X$ is a simple module $k(x)=A / \mathfrak{m}_{x}$ corresponding to a maximal ideal $\mathfrak{m}_{x} \subset A$. Moreover, $X$ is obviously the moduli space of its closed points, implying that the hull $H(k(x))$ of the deformation functor $\operatorname{Def}_{k(x)}: \underline{a}_{1} \rightarrow \underline{\text { Sets}}$, is the completion $\hat{A}_{\mathfrak{m}_{x}}$ of the local ring $O_{X, x}$ at the point $x \in X$. The regular functions $f$ of $X$, i.e. the sections of the structure sheaf $O_{X}$, are analytically determined by the family of Taylor series $\hat{f}_{x} \in \hat{A}_{\mathfrak{m}_{x}}$ at the different points $x \in X$, therefore by their (right multiplicative) actions on the hull of the different deformation functors $\operatorname{Def}_{k(x)}$.

This completion process, and the corresponding identification of a regular function $f$ on $X$ as a (multiplicative) operator in the $k$-vector space $A$ as well as in $\hat{A}_{x}$, for every $x \in X$, is going to replace the localization process of classical scheme theory.

Recall that to recover the affine ring $A$ from the scheme,

$$
\left(X, O_{X}\right)
$$

we are dependent upon the Zariski topology of $X$ and upon the sheaf property of $O_{X}$, both stemming from the process of localization in commutative rings. We find,

$$
A=H^{0}\left(X, O_{X}\right)
$$

Recall also that for noncommutative rings, the localization process functions only for Ore-sets, which are scarce. To obtain a good noncommutative theory we shall therefore have to change the notion of space, conserving the notion of points and incidences, but (for the moment) leaving out the topology. The notion of structure sheaf must therefore be modified.

Let $\underline{c}$ be any reasonable diagram of objects and morphisms in $\underline{C}$, and let $|\underline{c}|$ be the family of objects. We shall assume, for the rest of this paper, that 
for any $V_{i}, V_{j} \in|\underline{c}|$,

$$
\operatorname{dim}_{k} \operatorname{Ext}_{A}^{1}\left(V_{i}, V_{j}\right)<\infty .
$$

Let

$$
\pi: \underline{C} \rightarrow k-\bmod .
$$

be the obvious forgetful functor.

Assume first that $|\underline{c}|=\left\{V_{i}\right\}_{i=1}^{r}$ is a finite swarm. Let $H(|\underline{c}|)=\left(H_{i, j}\right)$ be the hull of the noncommutative deformation functor of this family of $A$-modules. To the diagram $\underline{c}$ we now associate, see $\S 3$, a subalgebra,

$$
O^{A}(\underline{c}, \pi) \subseteq\left(H_{i, j}(|\underline{c}|) \otimes_{k} \operatorname{Hom}_{k}\left(V_{i}, V_{j}\right)\right)
$$

of preobservables, together with a restriction of the canonical homomorphism $\eta$,

$$
\eta(\underline{c}): A \longrightarrow O^{A}(\underline{c}, \pi),
$$

such that $O^{A}(\underline{c}, \pi)$ acts on $\underline{c}$, extending the action of $A$. Moreover, if for all $i, \operatorname{dim}_{k} V_{i}<\infty$, the $O$-construction is a closure operator, i.e.

$$
O^{A}(\underline{c}, \pi) \simeq O^{O}(\underline{c}, \pi)
$$

Notice that we shall, abusing the notations, write $\otimes$ where one should have written $\hat{\otimes}$, i.e. when $H\left(V_{i}\right) \in \underline{\hat{a}}_{r}$ but $H\left(V_{i}\right) \notin \underline{a}_{r}$ and where we therefore have to work with complete tensor products.

To extend this $O$-construction to infinite swarms, we have to "sheafify" the $O$-construction, obtaining for every finite swarm $\underline{c}$ a smaller $k$-algebra, $\mathcal{O}(\underline{c}, \pi)$ containing the image of $\eta(\underline{c})$. This new $k$-algebra of observables has good functorial properties and may be extended to the permissible infinite swarms, see (3.15). The final noncommutative structure sheaf $\mathcal{O}_{\pi}$, a certain quotient of this $\mathcal{O}(-, \pi)$, see $\S 3$, is a presheaf of $k$-algebras on the ordered set of sub-swarms of a given swarm $\underline{c}$. We then proclaim,

Definitions 3.8 and 3.17 A permissible swarm $\underline{c}$ of $\underline{C}$ will be called a prescheme for $A$, if

$$
\eta(\underline{c}): A \longrightarrow \mathcal{O}(\underline{c}, \pi)
$$

is an isomorphism. If this is the case, $(\underline{c}, A)$ is called an affine prescheme and we shall refer to $A$ as the affine ring of this prescheme. The swarm $\underline{c}$ will be called a scheme for $A$, if

$$
\eta(\underline{c}): A \longrightarrow \mathcal{O}_{\pi}(\underline{c})
$$

is an isomorphism. If this is the case, $(\underline{c}, A)$ is called an affine scheme and we shall refer to $A$ as the affine ring of this scheme. 
In particular, if $\underline{c}$ is a finite swarm of $A$-modules, the pair,

$$
\left(\underline{c}, \mathcal{O}^{A}(\underline{c}, \pi)\right)
$$

is, by (3.7), an affine (noncommutative) prescheme. Thus, (ii) in the commutative scheme theory, is replaced by (iii) in the general case.

Notice that we are now talking about a scheme for $A$, not about the scheme for $A$. In fact it is easily seen that there may be several useful schemes for a given algebra $A$, depending upon what kind of properties of the algebra one would like to study.

The noncommutative algebraic geometry we propose, is concerned with these affine schemes, and their globalizations. The categorical properties of our universe $\underline{C}$ replace the topology, and the classical structure sheaf $O_{X}$ is replaced by the $\mathcal{O}_{\pi}$-construction.

As an example, let us consider the 0-dimensional case. If $A$ is a commutative $k$-algebra of finite $k$-dimension, then $A=\oplus_{i=1}^{r} O_{X, x_{i}}$ where $X=$ $\operatorname{Spec}(A)=\left\{x_{1}, \ldots, x_{r}\right\}$. In general, let $A$ be a finite dimensional $k$-algebra, $k$ algebraically closed, and $\mathcal{V}=\left\{V_{i}\right\}$ the (finite) family of all simple modules. We shall consider each module of this family as a point, and we shall consider the obvious forgetful functor $\pi: A$-mod $\rightarrow k$-mod. The local ring (or the infinitesimal neighbourhood) of a point $V_{i}$ of $\mathcal{V}$, the analogue of the completion $\hat{O}_{X, x}$ of the local ring $O_{X, x}$ of a closed point $x \in X=\operatorname{Spec}(A)$, is the algebra

$$
H\left(V_{i}\right) \otimes_{k} \operatorname{End}_{k}\left(V_{i}\right)
$$

where $H\left(V_{i}\right)$ is the hull of the deformation functor $\operatorname{Def}_{V_{i}}$. The affine ring $A$ is, however, no longer isomorphic to the sum of these local algebras.

Here is where the notion of noncommutative deformation enters. Let $H(\mathcal{V})=\left(H_{i, j}\right)$ be the hull of the noncommutative deformation functor of the family $\mathcal{V}=\left\{V_{i}\right\}$, see [10], then the infinitesimal interactions of the points of $\mathcal{V}$, translates into the components,

$$
H_{i, j}(\mathcal{V}) \otimes_{k} \operatorname{Hom}_{k}\left(V_{i}, V_{j}\right), i \neq j
$$

of the ring of observables $O(\mathcal{V}):=O(\mathcal{V}, \pi) . H_{i, j}(\mathcal{V})$ is, as a $H_{i, i}(\mathcal{V})-H_{j, j}(\mathcal{V})$ bi-module generated by a dual base of the tangent space $\operatorname{Ext}_{A}^{1}\left(V_{i}, V_{j}\right)$ of the point $V_{i}$ into the point $V_{j}$. There is a natural morphism of $k$-algebras,

$$
\eta: A \longrightarrow O(\mathcal{V}):=\left(H_{i, j}(\mathcal{V}) \otimes_{k} \operatorname{Hom}_{k}\left(V_{i}, V_{j}\right)\right)
$$

which, according to the Generalized Burnside Theorem is an isomorphism.

This is the Serre theorem, i.e. the analogue of (ii), in the 0-dimensional noncommutative algebraic geometry. The discrete swarm,

$$
\left(\mathcal{V}:=\left\{V_{i}\right\}_{i=1}^{r}, A\right)
$$

is a scheme for $A$. 
Notice that in the construction of $H(\mathcal{V})$ we only use the structure of the abelian category $\underline{C}$ (of $A$-modules) in which we consider our family of objects $\mathcal{V} . H(\mathcal{V})$ is therefore an invariant of the Morita equivalence class of $A$. To recover $A$, i.e. in the construction of the ring of observables, we must also know the dimensions of the different points $V_{i}$ of the noncommutative scheme $\underline{c}:=\mathcal{V}$, i.e. we must know the values of the forgetful functor $\pi$ on $\mathcal{V}$. However, as one easily shows, $H(\mathcal{V})$ is Morita equivalent to $A$.

- Now, to call something a geometry, one should certainly have the possibility of defining some kind of hierarchy among the geometrical subobjects, something like a quiver of incidences. Given a geometrical subobject we should at least be able to decide which points sit on the subvariety. In our case, if $\underline{c}$ is an affine scheme for $A$, the morphisms of $\underline{c}$ correspond to incidences among the points. Moreover, as we have seen in the 0-dimensional case discussed above, there may also be some infinitesimal incidences between the points $V_{i}$ and $V_{j}$, corresponding to a $k$-basis of $\operatorname{Ext}_{A}^{1}\left(V_{i}, V_{j}\right)$. And these are essential in the (re)construction of the affine ring of observables.

- To qualify as a geometry, a model should include a dynamical element, i.e. either a topology and a differential structure, including vectorfields, or something taking its place. This is, in our case, provided by a differential calculus induced by the deformation theory, see (3.32), where the basic notions are introduced.

- To be taken seriously, a noncommutative algebraic geometry must certainly include the classical algebraic geometry as a special case. To see that our model satisfies this condition, let $A$ be a commutative $k$-algebra of finite type. The points of the affine scheme $\operatorname{Spec}(A)$ may be identified with the members of the family of indecomposable modules $\mathcal{V}=\{A / \mathfrak{p}\}_{\mathfrak{p} \in \operatorname{Spec}(A)}$. We shall consider this family of $A$-modules together with the obvious canonical morphisms, obtaining a (usually infinite) diagram (really an ordered set) $\underline{c}=\operatorname{Spec}(A)$, of $A$-mod. Notice that $\operatorname{Spec}(A)$ as a set, contains the set of closed points, $\operatorname{Simp}(A)$, the simple A-modules, together with all the irreducible subvarieties of $\operatorname{Spec}(A)$, considered both as points in their own right, and as defining subschemes, with the corresponding points as their generic points. This induces a notion of incidence among different points in the geometry, just as we have done above. Recall, however, that in classical scheme theory, a scheme is the moduli space of its closed points, but not necessarily of the non-closed points. There is, in fact, a dichotomy between the set of closed points and the set of non-closed points, between the scheme and its Hilbert schemes.

In our noncommutative geometry, the general notion of scheme is an intermediate version, providing us with a set of points and incidences, such that all points are on equal footing, see section $\S 3$. 
These considerations lead us to the swarm of $A$-modules, $\operatorname{Simp}^{*}(A)$, consisting of $A$, the projective generator, and all the simple $A$-modules, together with the obvious incidences.

The embedding of the classical algebraic geometry (defined on an algebraically closed field $k$ ), into the proposed noncommutative algebraic geometry, is then taken care of by the following results,

Proposition 3.20 Let $A$ be any $k$-algebra of finite type, $k$ algebraically closed. Assume the natural homomorphism,

$$
\eta(\operatorname{Simp}(A), \pi): A \rightarrow \mathcal{O}(\operatorname{Simp}(A), \pi)
$$

is injective, then the canonical morphism of $k$-algebras

$$
\eta\left(\operatorname{Simp}^{*}(A), \pi\right): A \rightarrow \mathcal{O}_{\pi}\left(\operatorname{Simp}^{*}(A)\right)
$$

is an isomorphism, i.e. $\operatorname{Simp}^{*}(A)$ is a scheme for $A$.

Theorem 4.1 Let $A$ be any commutative $k$-algebra of finite type, $k$ algebraically closed. Then $\operatorname{Simp}^{*}(A)$ is a permissible swarm, and the canonical morphism of $k$-algebras

$$
\eta\left(\operatorname{Simp}^{*}(A), \pi\right): A \rightarrow \mathcal{O}_{\pi}\left(\operatorname{Simp}^{*}(A)\right)
$$

is an isomorphism, i.e. $\operatorname{Simp}^{*}(A)$ is a scheme for $A$.

Here topology and localizations comes back in. Using the Jacobson topology on $\operatorname{Simp}(A)$, we shall see that there is a natural way of localizing in noncommutative $k$-algebras, obtaining a structure presheaf $\mathcal{O}_{\pi}$ defined on this Jacobson topology, generalizing the commutative case.

We shall look at invariant theory, in this general setting and, in particular, we shall see that many problems of moduli in algebra, which cannot be treated in the classical framework of schemes, or of ringed spaces, have very satisfactory solutions expressible in the language of this generalized scheme theory, see section $\S 8$.

Now, a noncommutative version of differential geometry has been around for decades, spured by the needs of quantum mechanics and by the needs of invariant theory. In fact, there is a flora of proposed noncommutative geometries. The first ones were based on the notion of operator algebras. Von Neumann's work on quantum mechanics created a geometry where points, in some sense, were replaced by states or pure states in $\mathrm{C}^{*}$-algebras. Working on foliations, Connes has, in a most convincing way, developed a theory of quotient spaces, or orbit spaces, related to the theory of moduli, which transcends the classical geometry. However, the basic notion of space as a set of points with a topology and a structure defined by a structure sheaf, disappears in this model, see [3]. 
There are also purely algebraic attempts at the construction of a noncommutative geometry see e.g. [1], [14], [20], [2], [13], and [16]. The common aspect of these models have been that they do not include nonreduced schemes, and therefore cannot treat 0-dimensional schemes, and subsequently contain no infinitesimal theory. However, noncommutative geometry is a field in progress, and there are hopes for a future convergence of views, and a common ground for noncommutative algebraic geometry.

Earlier versions of this paper has appeared in the preprints [11, 12] and reference [10].

\section{Homological preparations}

\section{Exts and Hochschild cohomology}

Let $k$ be a (usually algebraically closed) field, and let $A$ be a $k$-algebra. Denote by $A$-mod the category of right $A$-modules and consider the exact forgetful functor

$$
\pi: A-\bmod \longrightarrow k \text {-mod }
$$

Given two $A$-modules $\mathrm{M}$ and $\mathrm{N}$, we shall always use the identification

$$
\sigma^{i}: \operatorname{Ext}_{A}^{i}(M, N) \simeq H H^{i}\left(A, \operatorname{Hom}_{k}(M, N)\right) \text { for } i=0,1,2,
$$

where $\operatorname{Hom}_{k}(M, N)$ is provided with the obvious left and right $A$-module structures. If $L_{*}$ and $F_{*}$ are $A$-free resolutions of $\mathrm{M}$ and $\mathrm{N}$ respectively, and if an element

$$
\xi \in \operatorname{Ext}_{A}^{1}(M, N)
$$

is represented by the Yoneda cocycle,

$$
\hat{\xi}=\left\{\xi_{n}\right\} \in \prod_{n} \operatorname{Hom}_{A}\left(L_{n}, F_{n-1}\right)
$$

then $\sigma^{1}(\xi)$ is gotten as follows. Let $\sigma$ be a $k$-linear section of the augmentation morphism

$$
\rho: L_{0} \longrightarrow M
$$

and let for every $a \in A$ and $m \in M, \sigma(m a)-\sigma(m) a=d_{0}(x)$. Put,

$$
\sigma^{1}(\hat{\xi})(a, m)=-\mu\left(\xi_{1}(x)\right)
$$

where

$$
\mu: F_{0} \longrightarrow N
$$

is the augmentation morphism of $F_{*}$. Then,

$$
\sigma^{1}(\hat{\xi}) \in \operatorname{Der}_{k}\left(A, \operatorname{Hom}_{k}(M, N)\right)
$$

and its class in $H H^{1}\left(A, \operatorname{Hom}_{k}(M, N)\right)$ equals $\sigma^{1}(\xi)$. 
Recall the spectral sequence associated to a change of rings. If $\pi: A \rightarrow B$ is a surjective homomorphism of commutative $k$-algebras, $M$ a $B$-module and $N$ an $A$-module, then $\operatorname{Ext}_{A}^{*}(M, N)$ is the abuttment of the spectral sequence given by,

$$
E_{2}^{p, q}=\operatorname{Ext}_{B}^{p}\left(M, \operatorname{Ext}_{A}^{q}(B, N)\right) .
$$

There is an exact sequence,

$$
0 \longrightarrow E_{2}^{1,0} \longrightarrow \operatorname{Ext}_{A}^{1}(M, N) \longrightarrow E_{2}^{0,1} \longrightarrow E_{2}^{2,0}
$$

which, for a $B$-module $N$, considered as an $A$-module, implies the exactness of

$$
\begin{aligned}
0 & \longrightarrow \operatorname{Ext}_{B}^{1}(M, N) \longrightarrow \operatorname{Ext}_{A}^{1}(M, N) \\
& \longrightarrow \operatorname{Hom}_{B}\left(M, \operatorname{Hom}_{B}\left(I / I^{2}, N\right)\right) \longrightarrow \operatorname{Ext}_{B}^{2}(M, N)
\end{aligned}
$$

where $I=\operatorname{ker} \pi$. The corresponding exact sequence,

$$
\begin{aligned}
0 & \longrightarrow \\
& \longrightarrow H^{1}\left(B, \operatorname{Hom}_{k}(M, N)\right) \longrightarrow H H^{1}\left(A, \operatorname{Hom}_{A \otimes A^{o p}}\left(I, \operatorname{Hom}_{k}(M, N)\right)\right.
\end{aligned}
$$

in the noncommutative case is induced by the sequence

$$
\begin{aligned}
0 \longrightarrow \operatorname{Der}_{k}\left(B, \operatorname{Hom}_{k}(M, N)\right) \longrightarrow \operatorname{Der}_{k}\left(A, \operatorname{Hom}_{k}(M, N)\right) \\
\quad \longrightarrow \operatorname{Hom}_{A \otimes A^{o p}}\left(I, \operatorname{Hom}_{k}(M, N)\right) .
\end{aligned}
$$

Notice that in general we do not know that the last morphism is surjective. This, however, is true if $B=A / \operatorname{Rad}(A)$, where $\operatorname{Rad}(A)$ is the radical of $A$, and $A$ is a finite dimensional, i.e. an artinian, $k$-algebra. In this case, $B$ is semisimple and the surjectivity above follows from the WedderburnMalcev theorem. Notice also that in the commutative case,

$$
\operatorname{Hom}_{A \otimes A^{o p}}\left(I, \operatorname{Hom}_{k}(M, N)\right) \simeq \operatorname{Hom}_{B}\left(I / I^{2}, \operatorname{Hom}_{B}(M, N)\right)
$$

as it must, since for $\phi \in \operatorname{Hom}_{A \otimes A^{o p}}\left(I, \operatorname{Hom}_{k}(M, N)\right), a \in A$, and $i \in I$, $a i=i a$, and therefore

$$
a \phi(i)=\phi(a i)=\phi(i a)=\phi(i) a, \quad \text { i.e. } \quad \phi(i) \in \operatorname{Hom}_{B}(M, N) .
$$

This implies that for $B=A / \mathfrak{p}, M=A / \mathfrak{p}, N=A / \mathfrak{q}$, where $\mathfrak{p} \subseteq \mathfrak{q}$ are (prime) ideals of $A$,

$$
\operatorname{Ext}_{A}^{1}(A / \mathfrak{p}, A / \mathfrak{q}) \simeq \operatorname{Hom}_{A}\left(\mathfrak{p} / \mathfrak{p}^{2}, A / \mathfrak{q}\right)
$$

and, in particular

$$
\operatorname{Ext}_{A}^{1}(A / \mathfrak{q}, A / \mathfrak{q}) \simeq \operatorname{Hom}_{A}\left(\mathfrak{q} / \mathfrak{q}^{2}, A / \mathfrak{q}\right)=N_{\mathfrak{q}},
$$

the normal bundle of $V(\mathfrak{q})$ in $\operatorname{Spec}(A)$. If $\mathfrak{q} \subset \mathfrak{p}$ and $\mathfrak{q} \neq \mathfrak{p}$ we find,

$$
\operatorname{Ext}_{A}^{1}(A / \mathfrak{p}, A / \mathfrak{q}) \simeq \operatorname{Ext}_{A / \mathfrak{q}}^{1}(A / \mathfrak{p}, A / \mathfrak{q}) .
$$


In $[7$, chapter 1$]$, we considered the cohomology of a category $\underline{c}$ with values in a bifunctor, i.e. in a functor defined on the category mor $\underline{c}$ of morphisms of $\underline{c}$. Recall that a morphism between the objects $\psi$ and $\psi^{\prime}$ is a commutative diagram,

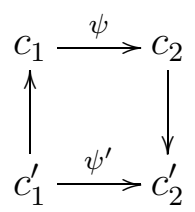

It is easy to see that this cohomology is an immediate generalization of the projective limit functor and its derivatives, or if one likes it better, the obvious generalization of the Hochschild cohomology of a ring. In fact, for every small category $\underline{c}$ and for every bifunctor,

$$
G: \underline{c} \times \underline{c} \longrightarrow \mathrm{Ab}
$$

contravariant in the first variable, and covariant in the second, one obtains a covariant functor

$$
G: \operatorname{mor} \underline{c} \longrightarrow \mathrm{Ab} \text {. }
$$

Consider now the complex

$$
D^{*}(\underline{c}, G)
$$

where

$$
D^{p}(\underline{c}, G)=\prod_{c_{0} \rightarrow c_{1} \cdots \rightarrow c_{p}} G\left(c_{0}, c_{p}\right),
$$

where the indices are strings of morphisms $\psi_{i}: c_{i} \rightarrow c_{i+1}$ in $\underline{c}$, and the differential,

$$
d^{p}: D^{p}(\underline{c}, G) \longrightarrow D^{p+1}(\underline{c}, G)
$$

is defined as usual,

$$
\begin{aligned}
& \left(d^{p} \xi\right)\left(\psi_{1}, \ldots, \psi_{i}, \psi_{i+1}, \ldots, \psi_{p+1}\right)=\psi_{1} \xi\left(\psi_{2}, \ldots, \psi_{p+1}\right) \\
& \quad+\sum_{i=1}^{p}(-1)^{i} \xi\left(\psi_{1}, \ldots, \psi_{i} \circ \psi_{i+1}, \ldots, \psi_{p+1}\right)+(-1)^{p+1} \xi\left(\psi_{1}, \ldots, \psi_{p}\right) \psi_{p+1}
\end{aligned}
$$

As shown in [7], the cohomology of this complex is the higher derivatives of the projective limit functor $\lim _{\text {mor } \underline{c}}^{(*)}$ applied to the covariant functor

$$
G: \operatorname{mor} \underline{c} \longrightarrow \mathrm{Ab} \text {. }
$$

This is the "Hochschild" cohomology of the category $\underline{c}$, denoted

$$
H^{*}(\underline{c}, G):=H^{*}\left(D^{*}(\underline{c}, G)\right) .
$$


Example 1.1 Let $\underline{c}$ be a multiplicative subset of a ring $\mathrm{R}$, considered as a category with one object, and let $\tilde{R}: \underline{c} \times \underline{c} \longrightarrow$ Ab be the functor, defined for $\psi, \psi^{\prime} \in \underline{c}$, by $\tilde{R}\left(\psi, \psi^{\prime}\right)=\psi^{*} \psi_{*}^{\prime}$, where $\psi^{*}$ is left multiplication on $R$ by $\psi$, and where $\psi_{*}^{\prime}$ is right multiplication on $R$ by $\psi^{\prime}$, then

$$
H^{0}(\underline{c}, \tilde{R})=\{\phi \in R \mid \phi \psi=\psi \phi \quad \text { for all } \psi \in \underline{c}\},
$$

i.e. the commutant of $\underline{c}$ in R.

Given a $k$-algebra $A$, and consider a subcategory $\underline{c}$ of the category of right $A$-modules. Let, as above $\pi: \underline{c} \rightarrow k$-mod be the forgetful-functor, and consider the bifunctor,

$$
\operatorname{Hom}_{\pi}: \underline{c} \times \underline{c} \longrightarrow k \text {-mod }
$$

defined by

$$
\operatorname{Hom}_{\pi}\left(V_{i}, V_{j}\right)=\operatorname{Hom}_{k}\left(V_{i}, V_{j}\right)
$$

Put,

$$
O_{0}(\underline{c}, \pi):=H^{0}\left(\underline{c}, \operatorname{Hom}_{\pi}\right) .
$$

It is clear that $O_{0}(\underline{c}, \pi)$ is a $k$-algebra, and that there is a canonical homomorphism of $k$-algebras,

$$
\eta_{0}(\underline{c}, \pi): A \longrightarrow O_{0}(\underline{c}, \pi)
$$

see $\S 3$.

Example 1.2 Let $A$ be a commutative $k$-algebra of finite type, $k$ algebraically closed, and let $\operatorname{Spec}(A)$ be the subcategory of $A$-mod consisting of the modules $A / \mathfrak{p}$, where $\mathfrak{p}$ runs through $\operatorname{Spec}(A)$, the morphisms being only the obvious ones. It is easy to see that the homomorphism

$$
\eta_{0}(\operatorname{Spec}(A), \pi): A \longrightarrow O_{0}(\operatorname{Spec}(A), \pi)
$$

identifies $A / \operatorname{Rad}(A)$ with $O_{0}(\operatorname{Spec}(A), \pi)$. If $\operatorname{Rad}(A)=0$, we even find an isomorphism,

$$
\eta_{0}\left(\operatorname{Simp}^{*}(A), \pi\right): A \simeq O_{0}\left(\operatorname{Simp}^{*}(A), \pi\right) .
$$

Here $\operatorname{Simp}^{*}(A)$ is the subcategory of $A$-mod where the objects are $A$ and the simple $A$-modules, $A / \mathfrak{m}$, i.e. the closed points of $\operatorname{Spec}(A)$, and where the morphisms are the obvious quotient morphisms $A \rightarrow A / \mathfrak{m} . \eta_{0}\left(\operatorname{Simp}^{*}(A), \pi\right)$ is, however not, in general, an isomorphism. This is easily seen when $A$ is a local $k$-algebra. To remedy this situation we shall in $\S 3$. introduce and study a generalization $O(\underline{c}, \pi)$ of $O_{0}(\underline{c}, \pi)$ defined in terms of the noncommutative deformation theory, see [10]. 


\section{Noncommutative deformations}

\section{The category $\underline{a}_{r}$ and liftings of modules}

Let $\underline{a}_{r}$ be the category of " $r$-pointed" artinian $k$-algebras. Recall that an object $R$ of $\underline{a}_{r}$ is a diagram of morphism of artinian $k$-algebras,

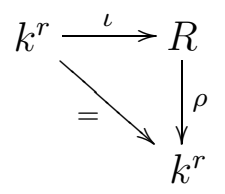

such that $\operatorname{Rad}(R):=\operatorname{ker} \rho$ is nilpotent, and such that,

$$
R / \operatorname{Rad}(R) \simeq \prod_{j=1}^{r} k_{j}, \quad k_{j} \simeq k .
$$

A morphism $\phi: R \rightarrow S$ of $\underline{a}_{r}$ is a morphism of such diagrams inducing the identity on $k^{r}$, implying that the induced map,

$$
k^{r} \simeq R / \operatorname{Rad}(R) \rightarrow S / \operatorname{Rad}(S) \simeq k^{r}
$$

is the identity. Pick idempotents $e_{i} \in k^{r} \subseteq R$ such that

$$
\sum_{i=1}^{r} e_{i}=1, \quad e_{i} e_{j}=0 \text { if } i \neq j .
$$

For every $(i, j)$, we shall consider the subspace $R_{i j}:=e_{i} R e_{j} \subseteq R$, and the pairing

$$
R_{i j} \otimes_{k} R_{j k} \rightarrow R_{i k}
$$

given in terms of the multiplication in $R$.

Let $R^{\prime}=\left(R_{i j}\right)$ be the matrix algebra, the elements of which are matrices of the form $\left(\alpha_{i j}\right)$ with $\alpha_{i j} \in R_{i j}, i, j=1, \ldots, r$. There is an obvious isomorphism of $k$-algebras

$$
\phi: R \rightarrow R^{\prime}
$$

defined by

$$
\phi(\alpha)=\left(e_{i} \alpha e_{j}\right) .
$$

identifying the sub $k$-algebra $k^{r}$ of $R$ with the algebra of diagonal $r \times r$ matrices. 


\section{The noncommutative deformation functor}

We are now ready to start the study of noncommutative deformations of the family $\mathcal{V}=\left\{V_{i}\right\}_{i=1}^{r}$. We shall assume,

$$
\operatorname{dim}_{k} \operatorname{Ext}_{A}^{1}\left(V_{i}, V_{j}\right)<\infty .
$$

Notice that the right $A$-module structure on the $V_{i}$ 's is defined by a homomorphism of $k$-algebras,

$$
\eta_{0}: A \rightarrow \oplus_{i=1}^{r} \operatorname{End}_{k}\left(V_{i}\right) .
$$

Given an object $\rho: R=\left(R_{i, j}\right) \rightarrow k^{r}$ of $\underline{a}_{r}$, consider the left $R$-module $\left(R_{i, j} \otimes_{k} V_{j}\right)$. $\rho$ defines a $k$-linear and left $R$-linear map,

$$
\rho(R):\left(R_{i, j} \otimes_{k} V_{j}\right) \rightarrow \oplus_{i=1}^{r} V_{i},
$$

inducing a homomorphism of $R$-endomorphism rings,

$$
\tilde{\rho}(R):\left(R_{i, j} \otimes_{k} \operatorname{Hom}_{k}\left(V_{i}, V_{j}\right)\right) \rightarrow \oplus_{i=1}^{r} \operatorname{End}_{k}\left(V_{i}\right) .
$$

Definition 2.1 The deformation functor

$$
\operatorname{Def}_{\mathcal{V}}: \underline{a}_{r} \rightarrow \underline{\text { Sets }}
$$

is defined for every $R \in \underline{a}_{r}$, as the set $\operatorname{Def}_{\mathcal{V}}(R) \in \underline{\text { Sets }}$ of isoclasses of homomorphisms of $k$-algebras,

$$
\left\{\eta^{\prime}: A \rightarrow\left(R_{i, j} \otimes_{k} \operatorname{Hom}_{k}\left(V_{i}, V_{j}\right)\right)\right\} / \sim
$$

such that,

$$
\tilde{\rho}(R) \circ \eta^{\prime}=\eta_{0}
$$

where the equivalence relation $\sim$ is defined by inner automorphisms in the $k$-algebra

$$
\operatorname{End}_{R}\left(\left(R_{i, j} \otimes_{k} V_{j}\right)\right)=\left(R_{i, j} \otimes_{k} \operatorname{Hom}_{k}\left(V_{i}, V_{j}\right)\right) .
$$

Any such isoclass $\tilde{\eta}^{\prime}$ will be called a deformation or a lifting of $V$ to $R$, and usually denoted $V_{R}$.

One easily proves that $\operatorname{Def}_{\mathcal{V}}$ has the same properties as the ordinary deformation functor.

Let $\pi: R \rightarrow S$ be a morphism of $\underline{a}_{r}$, such that $\operatorname{Rad}(R) \cdot \operatorname{ker} \pi=0$. Morphisms like this will be called small. If $V_{R} \in \operatorname{Def}_{V}(R)$ it is easy to see that $V_{S}:=S \otimes_{R} V_{R} \in \operatorname{Def}_{V}(S)$ and that $\bar{V}=\operatorname{ker}\left\{V_{R} \rightarrow S \otimes_{R} V_{R}\right\}$ is, as a left $R$-module, an $R / \operatorname{Rad}(R)=k^{r}$-module. Put $\operatorname{ker} \pi=\left(K_{i j}\right)$, then $\bar{V}=\left(\bar{V}_{i j}\right)$ where $\bar{V}_{i j}=K_{i j} \otimes_{k} V_{j}$. 
Consider now the $k$-vector spaces

$$
E_{i j}^{d}=\operatorname{Ext}_{A}^{d}\left(V_{i}, V_{j}\right)^{*}
$$

i.e. the dual $k$-vector spaces of $\operatorname{Ext}_{A}^{d}\left(V_{i}, V_{j}\right)$, and consider the $k$-algebra of matrices,

$$
T_{2}^{d}=\left(\begin{array}{ccc}
k & & 0 \\
& \ddots & \\
0 & & k
\end{array}\right)+\left(\epsilon_{i j} E_{i j}^{d}\right)
$$

where we assume all products of the $\epsilon_{i j}$ 's are equal to zero. Now let for every $i, j=1, \ldots, r$, and $d=1,2$,

$$
\left\{t_{i j}^{d}(\ell)\right\}_{\ell=1}^{e_{i j}^{d}}
$$

be a basis of $E_{i j}^{d}$, and let $\left\{\psi_{i j}^{d}(\ell)\right\}_{\ell=1}^{e_{i j}^{d}}$ be the dual basis. Thus $e_{i j}^{d}=\operatorname{dim}_{k} E_{i j}^{d}$. Consider the $k$-algebra

$$
T^{d}=\left(\begin{array}{ccc}
k & & 0 \\
& \ddots & \\
0 & & k
\end{array}\right)+\left(\widetilde{E}_{i j}^{d}\right)
$$

freely generated as matrix algebra by the generators $\left\{t_{i j}^{d}(\ell)\right\}_{\ell=1}^{e_{i j}^{d}}$. An element of $\widetilde{E}_{i j}^{d}$ is then a matrix where the elements are linear combinations of elements of the form:

$$
\begin{aligned}
\tau_{i j}=t_{i j_{1}}^{d}\left(l_{1}\right) \otimes t_{j_{1} j_{2}}^{d}\left(l_{2}\right) \otimes \cdots \otimes t_{j_{m-1} j_{m}}^{d}\left(l_{m}\right) \\
j=j_{m}, 1 \leq l_{s} \leq e_{j_{s-1} j_{s}}^{d}, 1 \leq j_{s} \leq r, m \geq 1
\end{aligned}
$$

of $E_{i j_{1}}^{d} \otimes E_{j_{1} j_{2}}^{d} \otimes \cdots \otimes E_{j_{m-1} j}^{d}$. Obviously

$$
T_{2}^{d}=T^{d} / \operatorname{Rad}\left(T^{d}\right)^{2}
$$

where $\operatorname{Rad}\left(T^{d}\right)$ is the two-sided ideal of $T^{d}$ generated by $\left(\widetilde{E}_{i j}^{d}\right)$.

Definition 2.2 For every object $R$ of $\underline{a}_{r}$, put

$$
T_{R}=\left(\operatorname{Rad}(R) / \operatorname{Rad}(R)^{2}\right)^{*}
$$

and call it the tangent space of $R$. 
Theorem 2.3 The functor Def $\mathcal{V}$ has a prorepresentable hull, or a formal moduli of $V, H(\mathcal{V})=: H \in \underline{\hat{a}}_{r}$, together with a formal versal family

$$
\tilde{V}=\left(H_{i, j} \otimes V_{j}\right) \in \lim _{n \geq 1} \operatorname{Def}_{\mathcal{V}}\left(H / \operatorname{Rad}(H)^{n}\right)
$$

such that the corresponding morphism of functors on $\underline{a}_{r}$,

$$
\rho: \operatorname{Mor}(H,-) \rightarrow \operatorname{Def}_{\mathcal{V}}
$$

is smooth and an isomorphism on the tangent level. Moreover, $H$ is uniquely determined by a set of matric Massey products defined on subspaces,

$$
D_{n} \subset \bigoplus_{p=2}^{n} \operatorname{Ext}^{1}\left(V_{i}, V_{j_{1}}\right) \otimes \cdots \otimes \operatorname{Ext}^{1}\left(V_{j_{p-1}}, V_{j}\right)
$$

with values in $\operatorname{Ext}^{2}\left(V_{i}, V_{j}\right)$.

Proof: See [10]. The proof of the existence of a prorepresentable hull for Def $\mathcal{V}$ can, of course, also be modeled on the classical proof of M. Schlessinger [17]. This has been carried out by Runar Ile, see [5].

This result may also be phrased as follows, see [7, §4],

Theorem 2.4 There is a morphism of proobjects of $\underline{\hat{a}}_{r}$,

$$
o: T^{2} \longrightarrow T^{1}
$$

determined by a sequence of well defined Massey products in $\operatorname{Ext}_{A}^{*}\left(V_{i}, V_{j}\right)$, such that

$$
H=T^{1} \otimes_{T^{2}} k
$$

is the prorepresenting hull of the deformation functor Def $_{\mathcal{V}}$.

\section{The $O$-construction}

For every deformation $V_{R} \in \operatorname{Def}_{\mathcal{V}}(R)$ there exists, by definition an, up to inner automorphisms, unique homomorphism of $k$-algebras,

$$
\eta_{V_{R}}: A \rightarrow \operatorname{End}_{R}\left(V_{R}\right)=\left(R_{i j} \otimes \operatorname{Hom}_{k}\left(V_{i}, V_{j}\right)\right) .
$$

Let $H:=H(\mathcal{V})$ be the formal moduli for $\mathcal{V}$, and let $\tilde{V}$ the formal versal family. 
Definition 2.5 The finite family of $\mathcal{V}=\left\{V_{i}\right\}_{i=1}^{r}$ will be called a swarm of $A$-modules if,

$$
\operatorname{dim}_{k} \operatorname{Ext}_{A}^{1}\left(V_{i}, V_{j}\right)<\infty .
$$

and if there exist a natural $A$-module structure on the formal versal family, i.e. a natural homomorphism,

$$
\eta: A \rightarrow \operatorname{End}_{H}(\tilde{V}),
$$

inducing all $\eta_{V_{R}}$. The $k$-algebra,

$$
O(\mathcal{V}):=\operatorname{End}_{H}(\tilde{V})=\left(H_{i j} \otimes \operatorname{Hom}_{k}\left(V_{i}, V_{j}\right)\right),
$$

will then be called the algebra of observables of the family of $A$-modules $\mathcal{V}$.

The following result, proved in [10], plays an important role in the construction of a noncommutative algebraic geometry in $\S 3$.

Theorem 2.6 (A generalized Burnside theorem) Let $A$ be a finite dimensional $k$-algebra, $k$ an algebraically closed field. Consider the family $\mathcal{V}=\left\{V_{i}\right\}_{i=1}^{r}$ of simple A-modules, then

$$
\eta(\mathcal{V}): A \rightarrow O(\mathcal{V})=\left(H_{i, j} \otimes \operatorname{Hom}_{k}\left(V_{i}, V_{j}\right)\right),
$$

is an isomorphism.

\section{Noncommutative modular deformations}

Let $V$ be any right $A$-module such that $\operatorname{dim}_{k} \operatorname{Ext}_{A}^{1}(V, V)<\infty$. Consider the formal moduli $H^{A}=: H$, the formal versal family $\tilde{V}=H \otimes V$, and the corresponding morphism of functors,

$$
\rho: \operatorname{Mor}_{\underline{a}_{r}}(H,-) \rightarrow \operatorname{Def}_{V} .
$$

We know that $\rho$ is not, in general, injective. However, $\mathrm{V}$ is also a right $A \otimes \operatorname{End}_{A}(V)$-module. As such it has a formal moduli $H^{A \text {,End }}$, and there is a natural $k$-algebra homomorphism, $H^{A} \rightarrow H^{A \text {,End }}$. Let $H_{0}^{A}$ be the unique maximal common quotient of $H^{A}$ and $H^{A \text {,End }}$. Using the same construction as in $[9, \S 2]$, we prove that the composition,

$$
\rho_{0}: \operatorname{Mor}_{\underline{a}_{r}}\left(H_{0},-\right) \rightarrow \operatorname{Mor}_{\underline{a}_{r}}(H,-) \rightarrow \operatorname{Def}_{V}
$$

is injective.

At the tangent level, the homomorphisms,

$$
H^{A} \rightarrow H^{A, \text { End }} \leftarrow H^{\text {End }},
$$

looks like the canonical homomorphisms,

$$
\operatorname{Ext}_{A}^{1}(V, V) \leftarrow \operatorname{Ext}_{A \otimes_{k} \text { End }}^{1}(V, V) \rightarrow \operatorname{Ext}_{\text {End }}^{1}(V, V) .
$$


Representing elements of the Ext-groups as derivations, it is easy to see that the two images are contained in the subspace $\operatorname{Ext}_{A}^{1}(V, V)^{\text {End }}$, respectively $\operatorname{Ext}_{\text {End }}^{1}(V, V)^{A}$. Therefore the tangent space of $H_{0}$ must be contained in the subspace of invariants under $\operatorname{End}_{A}(V)$ of the tangent space of $H$, $\operatorname{Ext}_{A}^{1}(V, V)^{\text {End }}$.

Example 2.7 Given any scheme $\underline{H}=\operatorname{Spec}(H)$, say the 2-dimensional affine space given by $H=k\left[x_{1}, x_{2}\right]$. We shall be interested in the (noncommutative) moduli space parametrizing subschemes of length 2 of $\underline{H}$. We may do this by simply considering a point in the space $\operatorname{Spec}(H)$ together with a tangent direction, i.e. the right $H$-module of the form,

$$
V=k\left[x_{1}, x_{2}\right] /\left(x_{1}^{2}, x_{2}\right),
$$

and compute the formal moduli of $V$.

Lemma 2.8 The formal moduli, $H(V)$ of the $H$-module $H /\left(x_{1}^{2}, x_{2}\right)$, is given as the completion of the $k$-algebra,

$$
\Omega=k<t_{1}, t_{2}, \omega_{1}, \omega_{2}>/\left(y_{1}, y_{2}\right)
$$

where

$$
y_{1}=\left[t_{1}, t_{2}\right]-t_{1}\left[\omega_{1}, \omega_{2}\right] \quad y_{2}=\left[t_{1}, \omega_{2}\right]-\left[t_{2}, \omega_{1}\right]-\omega_{1}\left[\omega_{1}, \omega_{2}\right],
$$

and where the family of left $\Omega$ - and right $H$-modules,

$$
\Omega \otimes_{k} k^{2}
$$

is defined by the actions of $x_{1}$ and $x_{2}$, given by,

$$
x_{1}=\left(\begin{array}{cc}
0 & t_{1} \\
1 & \omega_{1}
\end{array}\right), x_{2}=\left(\begin{array}{cc}
t_{2} & t_{1} \omega_{2} \\
\omega_{2} & t_{2}+\omega_{1} \omega_{2}
\end{array}\right)
$$

Proof: See [10]. Consider the obvious free resolution of $V:=H /\left(x_{1}^{2}, x_{2}\right)$ as an $H$-module,

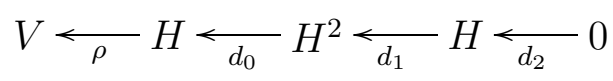

where we have,

$$
d_{0}=\left(x_{1}^{2}, x_{2}\right), d_{1}=\left(\begin{array}{c}
x_{2} \\
-x_{1}^{2}
\end{array}\right) .
$$

Consider the Yoneda complex, and pick a basis

$$
\left\{\hat{t}_{1}, \hat{t}_{2} ; \hat{\omega}_{1}, \hat{\omega}_{2},\right\}
$$


of $\operatorname{Ext}_{H}^{1}(V, V)$ represented by the morphisms of the diagram,

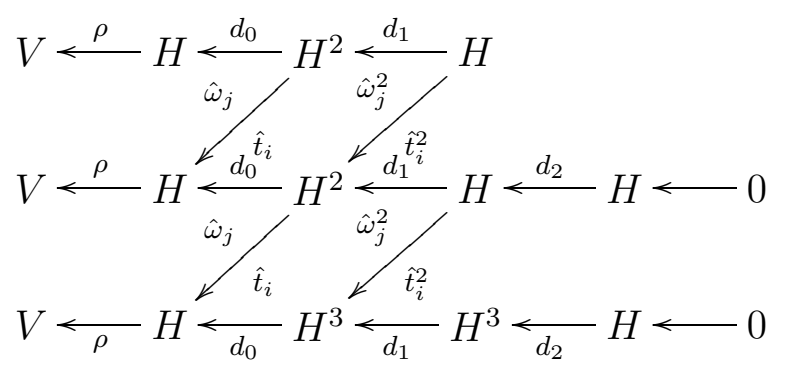

Here,

$$
\begin{aligned}
& \hat{t}_{1}=(1,0), \quad \hat{t}_{2}=(0,1) ; \\
& \hat{\omega}_{1}=\left(x_{1}, 0\right), \quad \hat{\omega}_{2}=\left(0, x_{1}\right)
\end{aligned}
$$

and,

and finally,

$$
\hat{t}_{1}^{2}=\left(\begin{array}{l}
0 \\
1
\end{array}\right), \hat{t}_{2}^{2}=\left(\begin{array}{c}
-1 \\
0
\end{array}\right)
$$

$$
\hat{\omega}_{1}^{2}=\left(\begin{array}{c}
0 \\
x_{1}
\end{array}\right), \hat{\omega}_{2}^{2}=\left(\begin{array}{c}
-x_{1} \\
0
\end{array}\right) .
$$

Using this it is easy to see that

$$
\hat{t}_{i} \cup \hat{t}_{i}=0, \hat{t}_{1} \cup \hat{t}_{2}=-\hat{t}_{2} \cup \hat{t}_{1}=\hat{y}_{1}
$$

and that

$$
\hat{t}_{1} \hat{\omega}_{2}^{2}=\hat{\omega}_{1} t_{2}^{2}=-\hat{y}_{2}, \hat{\omega}_{i} \hat{t}_{i}^{2}=0, \quad \hat{\omega}_{i} \hat{\omega}_{j}^{2}=0, \quad \hat{t}_{2} \hat{\omega}_{1}^{2}=\hat{\omega}_{2} \hat{t}_{1}^{2}=\hat{y}_{2},
$$

therefore

$$
-y_{2}=\hat{t}_{1} \cup \hat{\omega}_{2}=\hat{\omega}_{1} \cup \hat{t}_{2}=-\hat{t}_{2} \cup \hat{\omega}_{1}=-\hat{\omega}_{2} \cup \hat{t}_{1}, \quad \hat{\omega}_{i} \cup \hat{\omega}_{j}=\hat{t}_{i} \cup \hat{t}_{j}=0 .
$$

Now, consider the dual basis $\left\{t_{1}, t_{2} ; \omega_{1}, \omega_{2}\right\}$ generating the hull of the deformation functor $\operatorname{Def}_{k[\epsilon]}$, we find after a simple computation of the third order Massey products the formulas we want.

Notice that we just have to compute the tangent situation and check that our formulas give us a lifting of the quadratic relations and of the corresponding $H$-action, to know that our result holds.

Recall that the (commutative) Hilb $\mathbf{A}^{2}$ is the blow-up of $\left(\mathbf{A}^{2} \times \mathbf{A}^{2}\right) / \mathbf{Z}_{2}$ along the diagonal. By a simple computation one checks that the $k$-points of $\Omega$ form an open dense part of $\operatorname{Hilb}^{2} \mathbf{A}^{2}$ containing $V$. However, there are other simple representations of $\Omega$. The homomorphism,

$$
\left.\Omega \rightarrow k \mid t_{1}, t_{2}, \frac{\partial}{\partial t_{1}}, \frac{\partial}{\partial t_{2}}\right]
$$

mapping $\omega_{i}$ to $\partial / \partial t_{i}$, shows that $\left.k \mid t_{1}, t_{2}\right]$ is a simple representation of $\Omega$. 
Example 2.9 It is known that for any $A$-bimodule M,

$$
H H^{p}(A ; M)=\lim _{\{F \rightarrow A\}}{ }^{(p-1)} \operatorname{Der}_{k}(F, M), p \geq 2,
$$

where $\{F \rightarrow A\}$ denotes the category of $k$-free algebras $F$ above $A$. Moreover, the following sequence is exact,

$$
0 \rightarrow M^{A} \rightarrow M \rightarrow \operatorname{Der}_{k}(A, M) \rightarrow H H^{1}(A, M) \rightarrow 0 .
$$

Let $A=k<x_{1}, \ldots, x_{d}>$, be the free $k$-algebra on $d$ symbols, then we deduce,

$$
\operatorname{Ext}_{A}^{p}(N, M)=0, \text { for } p \geq 2,
$$

and for all right $A$-modules $N, M$. Given any simple $n$-dimensional $A$ representation $V$ we therefore find,

$$
\operatorname{dim}_{k} \operatorname{Ext}_{A}^{1}(V, V)=(d-1) n^{2}+1 .
$$

This shows that,

$$
H(V)=k\left\{\left\{t_{1}, \ldots, t_{r}\right\}\right\}, \quad r=(d-1) n^{2}+1 .
$$

This explains some results of [14], see [4], Theorem 23, Theorem 27 and Theorem 28.

The fact that for any simple $n$-dimensional representation (i.e. right $A$ module) $V, H(V)$ is the completion of a free $k$-algebra on $(d-1) n^{2}+1$ symbols, shows also that for all $d \geq 2$, and all $n \geq 2$, the natural homomorphism of $k$-algebras,

$$
A \rightarrow H(V) \otimes \operatorname{End}_{k}(V)
$$

is injective, a result we shall use in the next section, see (3.20).

\section{Noncommutative schemes}

\section{Swarms, trivializations and observables}

Let $\underline{C}$ be any abelian category with Massey products. The last proviso is satisfied if $\underline{C}$ has enough projectives, but there are other cases where Massey products exist even though projectives are scarce. See [8] and [18] for an exposition of the Massey product structure in the category of all $O_{X}$-modules, for $\mathrm{X}$ a scheme defined on some field $k$.

Let $\underline{c} \subseteq \underline{C}$ be a diagram, i.e. a family of objects $\mathcal{V}=\left\{V_{i}\right\}_{i}$ and morphisms between them. Put $|\underline{c}|:=\mathcal{V}$. We shall assume that all finite subfamilies of $|\underline{c}|:=\mathcal{V}$ are swarms, see (2.5). Assume moreover that there exists an exact and faithful functor,

$$
\pi: \underline{c} \longrightarrow k-\bmod
$$


Definition 3.1 Any such $\underline{c}$ will be called a swarm, and the functor $\pi$ will be called a trivialization of $\underline{c}$.

Example 3.2 The obvious example of this set up is the following: Let $A$ be any $k$-algebra, $k$ a field, put $\underline{C}=A$-mod and let

$$
\pi: A-\bmod \longrightarrow k-\bmod
$$

be the forgetful functor. Then $\pi$ will be a trivialization for any diagram

$$
\underline{c} \subseteq \underline{C}=A-\bmod
$$

Unless we specifically mention another choice of trivialization, this is the one we shall use in the sequel.

Fix the trivialization $\pi$ of $\underline{c} \subseteq \underline{C}$, and consider the functor,

$$
\operatorname{Hom}_{\pi}: \operatorname{mor} \underline{c} \longrightarrow k \text {-mod }
$$

defined for $\psi: c_{1} \rightarrow c_{2}$ in $\underline{c}$, by

$$
\operatorname{Hom}_{\pi}(\psi)=\operatorname{Hom}_{k}\left(\pi\left(c_{1}\right), \pi\left(c_{2}\right)\right),
$$

and refer to $\S 1$.

Definition $3.3 O_{0}:=O_{0}(\underline{c}, \pi):=H^{0}\left(\underline{c}, \operatorname{Hom}_{\pi}\right)$ is the $k$-algebra of immediate observables of $\underline{c}$.

It is clear that $O_{0}$ acts on each object $\pi(c) \in k$-mod, $c \in$ ob $\underline{c}$, in the sense that there is a canonical $k$-algebra homomorphism

$$
O_{0} \longrightarrow \operatorname{End}_{k}(\pi(c))
$$

such that the image diagram

$$
\pi(\underline{c}) \subseteq k-\bmod
$$

becomes a diagram of $O_{0}$-representations.

In the example above, we obtain for every diagram $\underline{c} \subseteq A$-mod, a $k$ algebra $O_{0}(\underline{c}, \pi)$ acting on every $A$-module in $\underline{c}$ such that $\underline{c}$ becomes a diagram of $O_{0}(\underline{c}, \pi)$-modules. Moreover there is a canonical homomorphism of $k$-algebras

$$
\eta_{0}: A \longrightarrow O_{0}(c, \pi)
$$

which is, in an obvious sense, a universal "extension" of the algebra $A$, by an algebra acting on the diagram $\underline{c}$. 
Since we have

$$
\underline{c} \subseteq O_{0}-\bmod
$$

and since the trivialization $\pi$ induces a trivialization,

$$
\pi_{0}: O_{0}-\bmod \longrightarrow k-\bmod
$$

we may repeat the construction of trivial observables. We obtain,

$$
O_{0}\left(\underline{c}, \pi_{0}\right)=O_{0}(\underline{c}, \pi)=O_{0}
$$

This implies that the operation of constructing trivial observables, is a closure operation.

Example 3.4 Consider any reduced commutative $k$-algebra $A$ of finite type. Recall from (1.2) that if $\underline{c}=\operatorname{Spec}(A)$, then

$$
\eta_{0}: A \longrightarrow O_{0}(\underline{c}, \pi)
$$

is an isomorphism, provided $k$ is algebraically closed.

Now, let $A$ be any associative $k$-algebra. Assume that $k$ is algebraically closed. Let $\operatorname{Simp}_{n}(A)$ be the set of simple $A$-modules of $k$-dimension $n$. Put, for any $n, \operatorname{Simp}_{\leq n}(A):=\cup_{s \leq n} \operatorname{Simp}_{s}(A)$, and put $\operatorname{Simp}_{<\infty}(A):=$ $\cup_{0 \leq s} \operatorname{Simp}_{s}(A)$. Let $\operatorname{Simp}^{*}(A)$ be the diagram consisting of the object $A$ and all the simples, $\operatorname{Simp}(A)$, together with all morphisms of right $A$-modules between $A$ and the simple modules.

Denote also by $\operatorname{Ind}(A)$ the full subcategory of $A$-mod defined by the indecomposable modules. It is easy to see that the canonical homomorphism

$$
\eta_{0}: A \longrightarrow O_{0}(\operatorname{Ind}(A), \pi)
$$

is an isomorphism when $A$ is right Noetherian. However, there is in general no isomorphism

$$
\eta_{0}: A \longrightarrow O_{0}\left(\operatorname{Simp}^{*}(A), \pi\right) .
$$

Notice that there is a generalized Zariski topology both on $\operatorname{Simp}(A)$ and $\operatorname{Ind}(A)$, due to Jacobson, defined as follows. Let $s \in A$ and consider the subset $D(s)$ of $\operatorname{Simp}(A)$ defined by the objects $\mathrm{V}$ for which $s$ is not a zero divisor. Obviously $D(s) \cap D\left(s^{\prime}\right)=D\left(s s^{\prime}\right)$, so $\{D(s)\}_{s \in A}$ is a basis for a topology.

The problem with $\operatorname{Ind}(A)$ is that it is too big, that the topology is too coarse, and that it has some unsatisfactory functorial properties. On the other hand, $\operatorname{Simp}^{*}(A)$ seem to be too small since, even for finite type $k$-algebras, the natural homomorphism $\eta_{0}: A \rightarrow O_{0}\left(\operatorname{Simp}^{*}(A), \pi\right)$ is far from an isomorphism. 
These problems stem from the trivial nature of the trivial observables. In the construction of $O_{0}$, we use only the trivial categorical structure of $A$-mod, restricted to $\underline{c}$. To get to the goal, we have to take into account the infinitesimal structure of the category $A$-mod, i.e. the abelian structure of $A$-mod and, in particular, the family of iterated extensions of the objects of $\underline{c}$.

The goal is to construct, for every diagram $\underline{c}$, an extension of $O_{0}(\underline{c}, \pi)$, which we shall denote $\mathcal{O}_{\pi}(\underline{c})$, and a factorization,

$$
A \stackrel{\eta}{\rightarrow} \mathcal{O}_{\pi}(\underline{c}) \stackrel{\rho}{\rightarrow} O_{0}(\underline{c}, \pi)
$$

of $\eta_{0}$. We shall show that $\mathcal{O}_{\pi}$, has good functorial properties, mimicking the notion of structure sheaf in commutative algebra, and providing us with a generalized, noncommutative, algebraic geometry. We shall be guided by the principles of the Introduction.

So consider a swarm $\underline{c}$ in $\underline{C}=A$-mod, together with the trivialization $\pi$. Assume first that $\underline{c}$ is finite. Let $|\underline{c}|=\left\{V_{i}\right\}_{i=1}^{r}$, be the family of objects, and construct the noncommutative formal moduli $H(|\underline{\mid}|)=\left(H_{i, j}\right)$ as in $\S 3$. Let $\tilde{V}=\left(H_{i, j} \otimes V_{j}\right)$ be the versal family and consider the $k$-algebra

$$
O(|\underline{c}|, \pi):=\operatorname{End}_{H}(\tilde{V})=\left(H_{i, j} \otimes \operatorname{Hom}_{k}\left(V_{i}, V_{j}\right)\right)
$$

and the $k$-algebra homomorphism,

$$
\eta(\mid \underline{c}): A \longrightarrow O(\mid \underline{c}, \pi)
$$

defined by the action of $A$ on $\tilde{V}$.

Recall that the noncommutative formal moduli is unique up to isomorphisms, and that having fixed a versal family, as a deformation, the action of $A$ on $\tilde{V}$ is unique up to isomorphisms. This means that for any other homomorphism

$$
\eta(\mid \underline{|c|})^{\prime}: A \longrightarrow O(|\underline{c}|, \pi)
$$

defining the same deformation, there exists an automorphism

$$
\omega \in O(|\underline{c}|, \pi)
$$

such that

$$
\eta(|\underline{c}|)^{\prime}=\omega \eta(|\underline{c}|) \omega^{-1} .
$$

Notice that $\omega$, as an element of $O(|\underline{c}|, \pi)$, is a unit.

Recall also that, for an artinian algebra $A$, the morphism $\eta(\operatorname{Simp}(A))$ is an isomorphism. 
Notice that, by definition of the terms, there is a canonical morphism of $k$-algebras,

$$
\rho_{0}: O(|\underline{c}|, \pi) \longrightarrow O_{0}(|\underline{c}|, \pi)
$$

which, together with $\eta$ and $\eta_{0}$ form a commutative diagram. Therefore $|\underline{c}|$ is, in an obvious sense, a family of $O(|\underline{c}|, \pi)$-modules. Notice also that if $\underline{c}_{1} \subseteq \underline{c}_{2}$ is an inclusion of swarms, there exist an, up to automorphisms, unique surjective homomorphism,

$$
h\left(\underline{c}_{1} \subseteq \underline{c}_{2}\right): H\left(\left|\underline{c}_{2}\right|\right) \longrightarrow H\left(\left|\underline{c}_{1}\right|\right)
$$

induced by the natural imbedding, $\underline{a}_{r_{1}} \rightarrow \underline{a}_{r_{2}}$, where $r_{i}, \quad i=1,2$ is the number of objects in $\underline{c}_{i}$. At the tangent level this morphism corresponds to the inclusion,

$$
\left(\operatorname{Ext}_{A}^{1}\left(V_{i}, V_{j}\right)\right)_{i, j=1, \ldots, r_{1}} \subseteq\left(\operatorname{Ext}_{A}^{1}\left(V_{i}, V_{j}\right)\right)_{i, j=1, \ldots, r_{2}} .
$$

Beware, this $k$-algebra homomorphism does not necessarily admit a section! The morphism $h\left(\underline{c}_{1} \subseteq \underline{c}_{2}\right)$ induces a unique homomorphism of $k$-algebras,

$$
o\left(\underline{c}_{1} \subseteq \underline{c}_{2}\right): O\left(\left|\underline{c}_{2}\right|, \pi\right) \rightarrow O\left(\left|\underline{c}_{1}\right|, \pi\right) .
$$

If $\underline{c}$ is infinite we shall later put,

$$
O(|\underline{c}|, \pi)=\lim _{\underline{c}_{0} \subseteq \underline{c}} O\left(\left|\underline{c}_{0}\right|, \pi\right)
$$

where $\underline{c}_{0}$ runs through all finite subdiagrams of $\underline{c}$. (This is possible, since $\underline{c}_{0}$ being fixed, we may choose bases $\left\{t_{i, j}(\ell)\right\}_{\ell}$ for each $\operatorname{Ext}_{A}^{1}\left(V_{i}, V_{j}\right)$, for all $V_{i}, V_{j} \in|\underline{c}|$, and construct in one sweep all the cup and Massey products defining all $\left.H\left(\left|\underline{c}_{0}\right|\right)\right)$. However, there is a problem related to the $A$-action, see (3.14) and the definition (3.15).

The $k$-algebra we are heading for is now a subquotient of $O(|\underline{c}|, \pi)$, singled out by the incidences of our geometry, i.e. by the morphisms,

$$
\phi_{i, j}: V_{i} \rightarrow V_{j}
$$

of our diagram.

Let $\Gamma(\underline{c})$ be the quiver corresponding to the swarm $\underline{c}$, i.e. a quiver with set of nodes equal to the set of objects of $\underline{c}$, and with arrows corresponding to the morphisms $\phi_{i, j}$ of $\underline{c}$. Notice that $\underline{c}$ is a diagram of $\underline{C}$ not a subcategory, therefore we do not require that the identities of the objects be morphisms of $\underline{c}$. Assume first that $\underline{c}$ is finite, and assume, for simplicity, that $\Gamma(\underline{c})$ (or $\underline{c}$ ) is connected. 
Corresponding to $\Gamma(\underline{c})$ there is the universal $k$-algebra $k[\Gamma(\underline{c})]$. Consider the obvious representation of $k[\Gamma(\underline{c})]$ on $V:=\oplus_{i=1}^{r} V_{i}$ mapping $k[\Gamma(\underline{c})]$ to the $k$-algebra end $(\underline{c})$, generated by the morphisms of $\underline{c}$, in $\operatorname{End}_{A}(V)$. Now $V$ is a $A \otimes k[\Gamma(\underline{c})]$ - module, and as such an $A$-module, as well as a $k[\Gamma(\underline{c})]$-module. We may consider the ordinary (noncommutative) deformation functors of this module, as $A \otimes k[\Gamma(\underline{c})]$ - module, as $A$-module, and as $k[\Gamma(\underline{c})]$-module. Let the formal moduli of these functors be, $H^{A, \Gamma}(V), \mathrm{H}(\mathrm{V})$ and $H^{\Gamma}(V)$, respectively. There are natural (non unique) morphisms,

$$
H^{\Gamma}(V) \rightarrow H^{A, \Gamma}(V) \leftarrow H(V)
$$

Recall from $\S 2$ that the modular, or prorepresentable, substratum $H^{A, \Gamma}(V)_{0}$ of $H^{A, \Gamma}(V)$ is the unique maximal quotient of $H^{A, \Gamma}(V)$ such that the composition,

$$
\operatorname{Mor}\left(H^{A, \Gamma}(V)_{0},-\right) \rightarrow \operatorname{Mor}\left(H^{A, \Gamma}(V),-\right) \rightarrow \operatorname{Def}_{A \otimes k[\Gamma]} V
$$

is injective. There is a universal deformation of $\mathrm{V}$ to $H^{A, \Gamma}(V)_{0}$, i.e. an action of $A \otimes k[\Gamma]$ on $H^{A, \Gamma}(V)_{0} \otimes V$, uniquely inducing all other modular deformations. Finally, let $H(\underline{c})$ be the unique common quotient of $H^{A, \Gamma}(V)_{0}$ and $H(V)$ defined by the induced morphism,

$$
H^{A, \Gamma}(V)_{0} \leftarrow H(V)
$$

Now, given any deformation $\xi_{S}$ of $\mathrm{V}$ to some $k$-algebra $S \in \underline{\hat{a}}_{1}$, denote by $\underline{m}$ the maximal ideal of $S$. Let $\tilde{S}$ be the $r$-pointed matrix $k$-algebra $\left(\tilde{S}_{i, j}\right)$ where $\tilde{S}_{i, i}=S$, on the diagonal, and $\tilde{S}_{i, j}=\underline{m}$ at the other places, i.e. for $i \neq j$. Clearly $\tilde{S}$ is in $\underline{\hat{a}}_{r}$, and the $i$-th row of the matrix $\left(\tilde{S}_{i, j} \otimes V_{j}\right)$ is

$$
\left(\underline{m} \otimes V_{1}\right) \oplus \cdots \oplus\left(S \otimes V_{i}\right) \oplus \cdots \oplus\left(\underline{m} \otimes V_{r}\right) \subseteq H(V) \otimes V .
$$

Let $v_{i} \in V_{i}$, and $a \in A$. The component of $\left(1 \otimes v_{i}\right) a$ in $S \otimes V_{j}$ for $i \neq j$ sits in $\underline{m} \otimes V_{j}$. This shows that $A$ acts on each line of the matrix $\left(\tilde{S}_{i, j} \otimes V_{j}\right)$, commuting with the left action of $\left(\tilde{S}_{i, j}\right)$, implying that $\left(\tilde{S}_{i, j} \otimes V_{j}\right)$ is, in a natural way, a noncommutative deformation of the family of right $A$ modules $|\underline{c}|$, to $\tilde{S}$. Therefore there is a morphism,

$$
\iota_{S}: H(|\underline{c}|) \longrightarrow \tilde{S}
$$

compatible with the specified deformations of right $A$-modules. This induces a morphism of $k$-algebras,

$$
\left(H_{i, j} \otimes \operatorname{Hom}_{k}\left(V_{i}, V_{j}\right)\right) \longrightarrow\left(\tilde{S}_{i, j} \otimes \operatorname{Hom}_{k}\left(V_{i}, V_{j}\right)\right)
$$


Since the right hand side $k$-algebra is a subalgebra of

$$
\operatorname{End}_{S}\left(S \otimes\left(\oplus_{i=1}^{r} V_{i}\right)\right)=\left(S \otimes \operatorname{Hom}_{k}\left(V_{i}, V_{j}\right)\right)
$$

we obtain a (non-unique) homomorphism of $k$-algebras,

$$
\kappa_{S}:\left(H_{i, j} \otimes \operatorname{Hom}_{k}\left(V_{i}, V_{j}\right)\right) \longrightarrow\left(S \otimes \operatorname{Hom}_{k}\left(V_{i}, V_{j}\right)\right)
$$

such that the action $\eta(|\underline{c}|)$ is mapped to the $A$-action on $S \otimes\left(\oplus_{i=1}^{r} V_{i}\right)$ defining the deformation $\xi_{S}$. In particular, for the versal deformation of $\mathrm{V}$ to $H(V)$, and for the versal $A$-action on $H(V) \otimes\left(\oplus_{i=1}^{r} V_{i}\right)$, there is a homomorphism of $k$-algebras,

$$
\kappa_{H(V)}:\left(H_{i, j} \otimes \operatorname{Hom}_{k}\left(V_{i}, V_{j}\right)\right) \longrightarrow\left(H(V) \otimes \operatorname{Hom}_{k}\left(V_{i}, V_{j}\right)\right)
$$

compatible with the actions. Notice that by construction of the terms involved, it is clear that $\kappa_{H(V)}$ is injective, and that $H(V)$ is generated by the images of the components $\iota_{i, j}: H_{i, j} \rightarrow H(V)$ of $\iota_{H(V)}$. Therefore we have the adjunction relation,

$$
\operatorname{Mor}_{\underline{a}_{r}}(H(|\underline{c}|), \tilde{S}) \simeq \operatorname{Mor}_{\underline{a}_{1}}(H(V), S) \text {. }
$$

Now, compose $\kappa_{H(V)}$ with the homomorphism induced by the quotient map $H(V) \rightarrow H(\underline{c})$ and get a $k$-algebra homomorphism,

$$
\kappa_{H(\underline{c})}:\left(H_{i, j} \otimes \operatorname{Hom}_{k}\left(V_{i}, V_{j}\right)\right) \longrightarrow\left(H(\underline{c}) \otimes \operatorname{Hom}_{k}\left(V_{i}, V_{j}\right)\right)
$$

Definition 3.5 The $k$-algebra of preobservables $O(\underline{c}, \pi)$ of the finite swarm $\underline{c}$, is the subalgebra of

$$
\left(H_{i, j}(|\underline{c}|) \otimes \operatorname{Hom}_{\pi}\left(V_{i}, V_{j}\right)\right)
$$

commuting, via the morphism,

$$
\kappa_{S}:\left(H_{i, j}(|\underline{c}|) \otimes \operatorname{Hom}_{\pi}\left(V_{i}, V_{j}\right)\right) \rightarrow\left(S \otimes \operatorname{Hom}_{k}\left(V_{i}, V_{j}\right)\right),
$$

induced by any surjective $k$-algebra homomorphism

$$
H(\underline{c}) \longrightarrow S
$$

with the corresponding representation of $k[\Gamma]$ in $\left(S \otimes \operatorname{Hom}_{k}\left(V_{i}, V_{j}\right)\right)$.

It is clear that $O(\underline{c}, \pi)$ is uniquely defined, up to isomorphisms, and that $\eta(|\underline{c}|)$ induces a homomorphism of $k$-algebras,

$$
\eta(\underline{c}): A \longrightarrow O(\underline{c}, \pi)
$$


Remark 3.6 Let $A$ be a finite type $k$-algebra, and let $\{\phi\}$ be the diagram defined by the canonical homomorphism,

$$
\phi: A \rightarrow k(x)
$$

of $A$ onto its closed point $k(x)$. The tangent space of $H:=H(|\{\phi\}|)$ is,

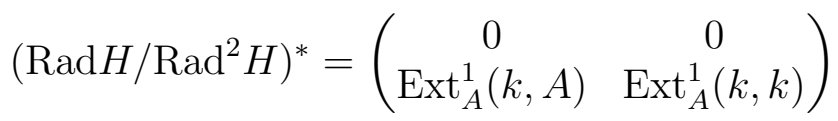

and the tangent space of $H(\{\phi\})$ looks like

$$
\left(\operatorname{Rad} H(\{\phi\}) / \operatorname{Rad}^{2} H(\{\phi\})\right)^{*}=\left(\begin{array}{cc}
0 & 0 \\
\operatorname{Ext}_{A}^{1}(k, A)^{\phi} & \operatorname{Ext}_{A}^{1}(k, k)
\end{array}\right),
$$

where $\operatorname{Ext}_{A}^{1}(k, A)^{\phi}$ is the kernel of $\phi_{*}: \operatorname{Ext}_{A}^{1}(k, A) \rightarrow \operatorname{Ext}_{A}^{1}(k, k)$. The morphism

$$
O(|\{\phi\}|, \pi) \rightarrow\left(\begin{array}{cc}
H(\{\phi\}) \otimes_{k} \operatorname{End}_{k}(A) & H(\{\phi\}) \otimes_{k} \operatorname{Hom}_{k}(A, k) \\
H(\{\phi\}) \otimes_{k} \operatorname{Hom}_{k}(k, A) & H(\{\phi\})
\end{array}\right)
$$

maps an element

to an element of the same form,

$$
\alpha=\left(\begin{array}{cc}
\alpha_{1,1} & 0 \\
\alpha_{2,1} & \alpha_{2,2}
\end{array}\right) \in O(|\{\phi\}|, \pi)
$$

$$
\tilde{\alpha}=\left(\begin{array}{cc}
\alpha_{1,1} & 0 \\
\tilde{\alpha}_{2,1} & \alpha_{2,2}
\end{array}\right) \in\left(\begin{array}{cc}
H(\{\phi\}) \otimes_{k} \operatorname{End}_{k}(A) & H(\{\phi\}) \otimes_{k} \operatorname{Hom}_{k}(A, k) \\
H(\{\phi\}) \otimes_{k} \operatorname{Hom}_{k}(k, A) & H(\{\phi\})
\end{array}\right) .
$$

Moreover a versal lifting of $\phi_{1,2}$ has the form,

$$
\Phi=\left(\begin{array}{cc}
0 & \Phi_{1,2} \\
\Phi_{2,1} & \Phi_{2,2}
\end{array}\right) \in\left(\begin{array}{cc}
H(\{\phi\}) \otimes_{k} \operatorname{End}_{k}(A) & H(\{\phi\}) \otimes_{k} \operatorname{Hom}_{k}(A, k) \\
H(\{\phi\}) \otimes_{k} \operatorname{Hom}_{k}(k, A) & H(\{\phi\})
\end{array}\right) .
$$

Suppose now that,

$$
\Phi \tilde{\alpha}=\tilde{\alpha} \Phi
$$

then, in particular,

$$
\Phi_{1,2} \tilde{\alpha}_{2,1}=0,
$$

which implies that $\tilde{\alpha}_{2,1}=0$, and then

$$
\Phi_{1,2} \alpha_{2,2}=\alpha_{1,1} \Phi_{1,2} \text {. }
$$

Since $\alpha_{2,2}=: \alpha_{x} \in H_{2,2}=\hat{A}_{\{x\}}$ is the obvious multiplication endomorphism, and since $\Phi_{1,2}$ reduces to the obvious completion map, $\rho_{x}: A \rightarrow H_{2,2}=\hat{A}_{\{x\}}$ we find that $\alpha \in O(\{\phi\}, \pi)$ if

$$
\tilde{\alpha}_{2,1}=0, \alpha_{1,1} \rho_{x}=\rho_{x} \alpha_{x}
$$

for some $\alpha_{x} \in \hat{A}_{\{x\}}$, and,

$$
\Phi_{2,1} \alpha_{1,1}=\alpha_{x} \Phi_{2,1} .
$$




\section{$O$ is a closure operation}

The most important property of the $O$-construction is a kind of functoriality (up to isomorphisms) and the closure property, given by the following result:

Theorem 3.7 Let $\psi: A \rightarrow B$ be a k-algebra homomorphism, and let $\underline{c}$ be a finite swarm of $A-B$-modules. Consider the $O$-constructions, $O^{A}(\underline{c}, \pi)$, resp. $O^{B}(\underline{c}, \pi)$.

a) Assume the natural morphism,

$$
\psi_{*}: H^{A, \Gamma}(V) \rightarrow H^{B, \Gamma}(V)_{0}
$$

induces a surjective homomorphism

$$
\psi_{*}: H^{A}(\underline{c}) \rightarrow H^{B}(\underline{c}) .
$$

Then there exists an, up to isomorphisms, unique extension of $\psi$, i.e. a commutative diagram,

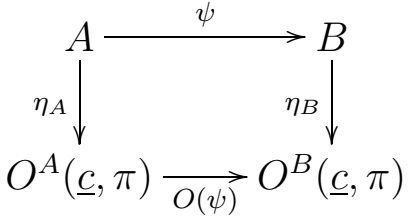

b) There is a natural isomorphism,

$$
O\left(\eta_{A}\right): O^{A}(\underline{c}, \pi) \rightarrow O^{O^{A}}(\underline{c}, \pi)
$$

implying that the $O$-construction is a closure operation.

Proof: Let the noncommutative formal moduli of the family of $B$-modules $\left\{V_{i}\right\}=|\underline{c}|$, considered as $A$ - and $B$-modules be $H^{A}$ resp. $H^{B}$. Since the versal family of $B$-modules $\left(H_{i, j}^{B} \otimes V_{j}\right)$ is also a family of $A$-modules, there is a morphism, $H^{A} \rightarrow H^{B}$ inducing the morphism of families of $A$-modules, $\left(H_{i, j}^{A} \otimes V_{j}\right) \rightarrow\left(H_{i, j}^{B} \otimes V_{j}\right)$ consistent with the induced $A$-module structure on the latter. In the same way we find that there exists a morphism of the formal moduli

$$
H^{A, \Gamma}(V) \longrightarrow H^{B, \Gamma}(V)
$$

of $V=\oplus_{i=1}^{r} V_{i}$ as a $A \otimes k[\Gamma(\underline{c})]$-module, resp. a $B \otimes k[\Gamma(\underline{c})]$-module, consistent with the families. By assumption, the above homomorphism induces a surjection

$$
\psi_{p}^{*}: H^{A}(\underline{c}) \longrightarrow H^{B}(\underline{c})
$$


By definition of $O$, we have a commutative diagram,

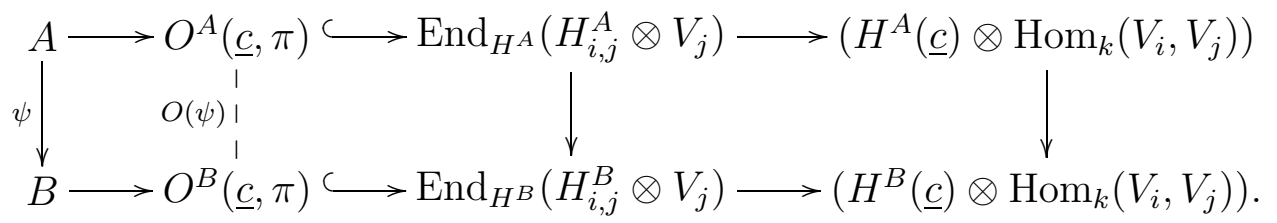

where, $O^{A}(\underline{c}, \pi)$ and $O^{B}(\underline{c}, \pi)$ are the commutants of the actions of $k[\Gamma(\underline{c})]$ in $\left(S \otimes \operatorname{Hom}_{k}\left(V_{i}, V_{j}\right)\right)$, for all quotients $S$ of $H^{A}(\underline{c})$, respectively of $H^{B}(\underline{c})$. The surjectivity of $\psi_{*}$, together with the commutativity of the diagram defines the morphism $O(\psi)$, and proves (a).

To prove (b), we just have to observe that $O^{A}$ acts on the $H^{A}$-family $\left(H_{i, j}^{A} \otimes V_{j}\right)$, consistent with the action of $A$ via $\eta_{A}$, and that $O \otimes k[\Gamma(\underline{c})]$ acts on the $H^{A}(\underline{c})$-family $\left(H^{A}(\underline{c}) \otimes V\right)$ consistent with the $A$-action via the obvious composition,

$$
\eta(V): A \longrightarrow\left(H^{A}(\underline{c}) \otimes \operatorname{Hom}_{k}\left(V_{i}, V_{j}\right)\right) .
$$

Therefore there must exist morphisms,

$$
\begin{gathered}
\left(H_{i, j}^{O^{A}}\right) \stackrel{\mu}{\longrightarrow}\left(H_{i, j}^{A}\right) \\
H^{O^{A}, \Gamma}(V) \stackrel{\mu(\underline{c})}{\longrightarrow} H^{A, \Gamma}(V)
\end{gathered}
$$

consistent with the obvious families. Moreover the composed morphisms

$$
\begin{gathered}
\left(H_{i, j}^{A}\right) \longrightarrow\left(H_{i, j}^{O^{A}}\right) \stackrel{\mu}{\longrightarrow}\left(H_{i, j}^{A}\right) \\
H^{A, \Gamma}(V) \longrightarrow H^{O^{A}, \Gamma}(V) \stackrel{\mu_{p}}{\longrightarrow} H^{A, \Gamma}(V)
\end{gathered}
$$

must be surjections inducing injections on the tangent spaces. Let us show that $\mu(\underline{c})$ induces a surjective homomorphism,

$$
H^{O^{A}}(\underline{c}) \stackrel{\mu_{0}}{\rightarrow} H^{A}(\underline{c}) .
$$

Consider the diagram,

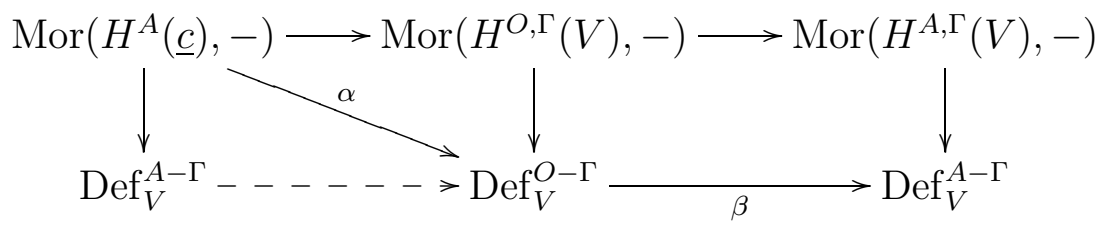

Define $\alpha$ by the composition, i.e. by considering the canonical $O \otimes k[\Gamma]$ structure on $H^{A}(\underline{c}) \otimes V$, and tensorization. Since the composition of $\alpha$ and $\beta$ is injective, $\alpha$ is injective. 
But this implies, by definition, and by the the unicity of the modular substratum, that the surjective homomorphism

$$
\mu_{0}: H^{O, \Gamma}(V) \longrightarrow H^{A}(\underline{c})
$$

induces a surjective homomorphism,

$$
\mu_{0}(\underline{c}): H^{O, \Gamma}(V)_{0} \longrightarrow H^{A}(\underline{c})
$$

and therefore also a surjection,

$$
\mu_{0}: H^{O}(\underline{c}) \longrightarrow H^{A}(\underline{c})
$$

Consider now the commutative diagram,

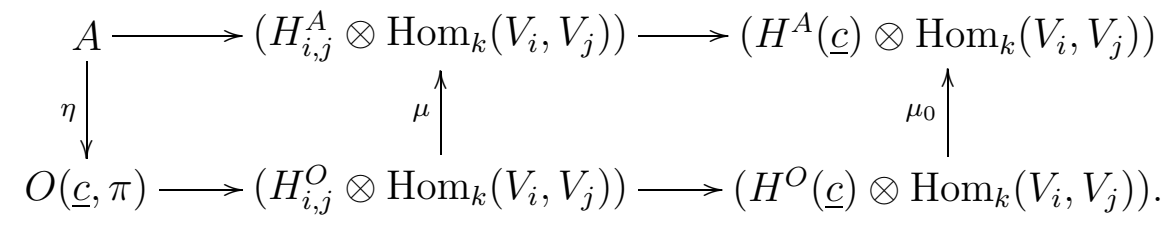

Since $\mu_{0}$ is consistent with the actions of $k[\Gamma]$, we obtain a cosection,

$$
\mu: O^{O}(\underline{c}, \pi) \longrightarrow O^{A}(\underline{c}, \pi)
$$

of the morphism,

$$
\eta_{1}: O^{A}(\underline{c}, \pi) \longrightarrow O^{O}(\underline{c}, \pi) .
$$

Now use (a) for the case $\psi=\mu$. Let us put $A^{\prime}=O^{O}(\underline{c}, \pi), B^{\prime}=O^{A}(\underline{c}, \pi)$. Since the composition of $\mu \circ \eta_{1}$ is the identity, the homomorphism,

$$
H^{A^{\prime}}(\underline{c}) \rightarrow H^{B^{\prime}}(\underline{c})
$$

must be surjective. We therefore obtain a commutative diagram,

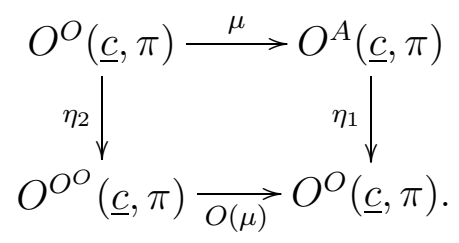

Since, by construction, the composition $\eta_{2} \circ O(\mu)$ is an isomorphism, $\mu$ must be injective, therefore an isomorphism, proving (b). 


\section{Noncommutative preschemes}

Definition 3.8 Let $A$ be any $k$-algebra. A finite swarm $\underline{c}$ of $A$-modules is called a prescheme for $A$, if the morphism

$$
\eta(\underline{c}, \pi): A \longrightarrow O(\underline{c}, \pi)
$$

is an isomorphism. In this case we shall refer to Aas the affine $k$-algebra of $\underline{c}$.

Corollary 3.9 Any finite swarm, $\underline{c}$, of right A-modules is a prescheme for $O^{A}(\underline{c}, \pi)$. In particular, if $\underline{c}$ is a diagram of finite dimensional $k$-vector spaces, then $\underline{c}$ is a swarm of right modules over $A=O^{k}(\underline{c}, \pi)=O_{0}(\underline{c}, \pi)$, and, as such, a prescheme for $A$.

Proof: This follows from the isomorphism

$$
O\left(\eta_{A}\right): O^{A}(\underline{c}, \pi) \rightarrow O^{O^{A}}(\underline{c}, \pi)
$$

of (3.7).

Example 3.10 According to the Generalized Burnside Theorem, if $A$ is any finite dimensional $k$-algebra, $k$ algebraically closed, the family of simple $A$-modules $V$ form a (0-dimensional) prescheme for $A$. In particular, if $\Lambda$ is a finite partially ordered set, then the set of nodes of $\Lambda$, considered as the set of simple $k[\Lambda]$-modules, is a scheme for $k[\Lambda]$.

Example 3.11 Consider the following trivial example where $\mathrm{A}=\mathrm{k}$ is a field, $V_{1}=k^{2}$ and $V_{2}=k$, and $\underline{c}$ is given by the diagram of right-modules,

$$
V_{1} \stackrel{\phi}{\longrightarrow} V_{2}
$$

where $\phi$ is the second projection. Obviously all Ext's vanish, so that

$$
\begin{gathered}
H(|\underline{c}|)=\left(\begin{array}{cc}
k & 0 \\
0 & k
\end{array}\right) \\
\left(H_{i, j} \otimes V_{j}\right)=\left(\begin{array}{cc}
V_{1} & 0 \\
0 & V_{2}
\end{array}\right)
\end{gathered}
$$

Moreover $H(\underline{c})=k$, and the maximal ideal $\underline{m} \subset H(\underline{c})$ is zero. Therefore,

$$
\bar{H}(\underline{c})=\left(\begin{array}{ll}
k & 0 \\
0 & k
\end{array}\right) \quad \text { and } \quad\left(\bar{H}(\underline{c})_{i, j} \otimes V_{j}\right)=\left(\begin{array}{cc}
V_{1} & 0 \\
0 & V_{2}
\end{array}\right)
$$


Therefore $O(\underline{c}, \pi)$ is the commutant algebra of the subalgebra,

$$
\operatorname{end}(\underline{c}) \subseteq\left(\begin{array}{ll}
\operatorname{Hom}_{k}\left(V_{1}, V_{1}\right) & \operatorname{Hom}_{k}\left(V_{1}, V_{2}\right) \\
\operatorname{Hom}_{k}\left(V_{2}, V_{1}\right) & \operatorname{Hom}_{k}\left(V_{2}, V_{2}\right)
\end{array}\right)
$$

generated by $\phi$, in the sub $k$-algebra,

$$
\left(\begin{array}{cc}
\operatorname{Hom}_{k}\left(V_{1}, V_{1}\right) & 0 \\
0 & \operatorname{Hom}_{k}\left(V_{2}, V_{2}\right)
\end{array}\right)
$$

which is easily seen to be equal to,

$$
O_{0}(\underline{c}, \pi)=\left(\begin{array}{ll}
k & 0 \\
k & k
\end{array}\right) .
$$

Now it is equally easy to see that $V_{1}$ identifies with the second line of

$$
O(\underline{c}, \pi)=\left(\begin{array}{ll}
k & 0 \\
k & k
\end{array}\right)
$$

as a right $O(\underline{c}, \pi)$-module, and is therefore projective, and that $V_{2}$ identifies with the second simple module of $O(\underline{c}, \pi)$. Trivial calculation gives that

$$
\operatorname{Ext}_{O}^{1}\left(V_{i}, V_{j}\right)=0, \forall i, j \text {. }
$$

Put $O:=O(\underline{c}, \pi)$. If we consider $\underline{c}$ as a diagram of $O$-modules, and repeat the $O$-construction, we therefore obtain $O^{O}=: O^{(2)}(\underline{c}, \pi)=O(\underline{c}, \pi)$, implying that the diagram $\underline{c}$ is a prescheme for $O$, as it must be, see (3.9). Since the $O(\underline{c}, \pi)$ we have found above is the algebra of the $\left(A_{1}\right)$ diagram,

$$
0^{1} \leftarrow \circ^{2}
$$

we find that as $O$-modules, the discrete diagram $\mathcal{V}=\{k(1), k(2)\}$ consisting of the two simple $O$-modules is also a prescheme for $O$. Notice, however, that as family of $k$-vector spaces $\mathcal{V}$ is a prescheme for $k^{2}$.

Example 3.12 That the claim of the Corollary (3.9) is not obvious, is seen by classifying the simple schemes of the form,

$$
\Gamma=\bigcap_{0}^{\phi}
$$

Let $\underline{c}=\{\phi: V \rightarrow V\}$ be a diagram of $k$-vector spaces. Assume $\operatorname{dim}_{k} V=3$, then according to the Jordan decomposition of $\phi$, we obtain the following $k$-algebras $A=O(\underline{c}, \pi)$ with $\underline{c}$ as affine scheme, 
(1) $\phi$ has 3 different eigenvalues, then $A=k^{3}$

(2) $\phi$ has only two different eigenvalues, then $A=k \times \operatorname{End}_{k}\left(k^{2}\right)$ or $A=$ $k \times k[\epsilon]$

(3) $\phi$ has all eigenvalues equal, then $A=\operatorname{End}_{k}\left(k^{3}\right)$ or $A=k[x] /\left(x^{3}\right)$ or

$$
A=\left(\begin{array}{cc}
k & k \cdot \epsilon_{1,2} \\
k \cdot \epsilon_{2,1} & k\left[\epsilon_{2,2}\right]
\end{array}\right)
$$

where we have the following relations,

$$
\epsilon_{1,2} \cdot \epsilon_{2,1}=0, \epsilon_{1,2} \cdot \epsilon_{2,2}=0, \epsilon_{2,2} \cdot \epsilon_{2,1}=0, \epsilon_{2,1} \cdot \epsilon_{1,2}=\epsilon_{2,2}
$$

The last algebra is evidently artinian with two simple representations, both of dimension 1. Therefore it has an affine scheme consisting of the discrete diagram consisting of those two $A$-modules. As such, it has infinitesimal incidences given by the family $\left\{\epsilon_{i, j}\right\}$. By (3.9) $A$ is also the affine algebra of the scheme $\underline{c}$, with quiver $\Gamma$.

Warning. NB! According to Example (3.4) if $A$ is any reduced finite type commutative $k$-algebra, and if $k$ is algebraically closed, $A \simeq O^{k}(\operatorname{Spec}(A), \pi)$. However $\operatorname{Spec}(A)$ is usually infinite. Therefore we cannot conclude from Corollary (3.9), that $\operatorname{Spec}(A)$ is a prescheme for $A$. In fact, $\operatorname{Spec}(A)$ is, in general, not a prescheme for $A$, since $O(\operatorname{Spec}(A), \pi)$ may well be noncommutative.

Example 3.13 Consider the special case of Remark (3.6), where $A$ is a local $k$-algebra, and $\phi: A \rightarrow k$ is the canonical homomorphism of $A$ onto its residue field. Since there is a surjective homomorphism,

$$
H(\{\phi\}) \longrightarrow H_{2,2}=\hat{A} \text {. }
$$

and since the completion map $\rho_{x}$ defines a lifting,

$$
\Phi_{1,2}: \hat{A} \otimes A \rightarrow H_{0}(\{\phi\}) \otimes k=\hat{A}
$$

of $\phi$, we observe that $\alpha_{1,1}$ is the right multiplication by some element $a=$ $\alpha_{1,1}(1) \in A$. Moreover $\alpha_{x}=\rho_{x}(a)$ and the condition

$$
\Phi_{2,1} \alpha_{1,1}=\alpha_{x} \Phi_{2,1}
$$

is automatically satisfied, since $\Phi_{1,2}$ is $A$-linear. Therefore,

$$
O(\{\phi\}, \pi)=\left\{\left(\begin{array}{cc}
\alpha & 0 \\
\alpha_{2,1} & \alpha_{x}
\end{array}\right) \mid \alpha \in A, \alpha_{x}=\rho_{x}(\alpha), \alpha_{2,1} \in H_{2,1} \otimes \operatorname{Hom}_{k}(k, A)\right\} .
$$

In particular, the natural morphism

$$
\eta: A \rightarrow O(\{\phi\}, \pi)
$$

is injective, and an isomorphism provided $\operatorname{Ext}_{A}^{1}(k, A)^{\phi}=\operatorname{Ext}_{A}^{1}(k, A)$. 


\section{Functoriality}

The $k$-algebra of preobservables of a diagram of modules is not, however, properly functorial with respect to inclusions between diagrams. The problem is the following: Let $\underline{c}_{0} \subseteq \underline{c}$ be a finite subdiagram. There is a corresponding inclusion of quivers, $\Gamma_{1}:=\Gamma\left(\underline{c}_{1}\right) \subseteq \Gamma(\underline{c})=$ : $\Gamma$, inducing a homomorphism of $k$-algebras,

$$
k\left[\Gamma\left(\underline{c}_{1}\right)\right] \longrightarrow k[\Gamma(\underline{c})] .
$$

Consider the diagram,

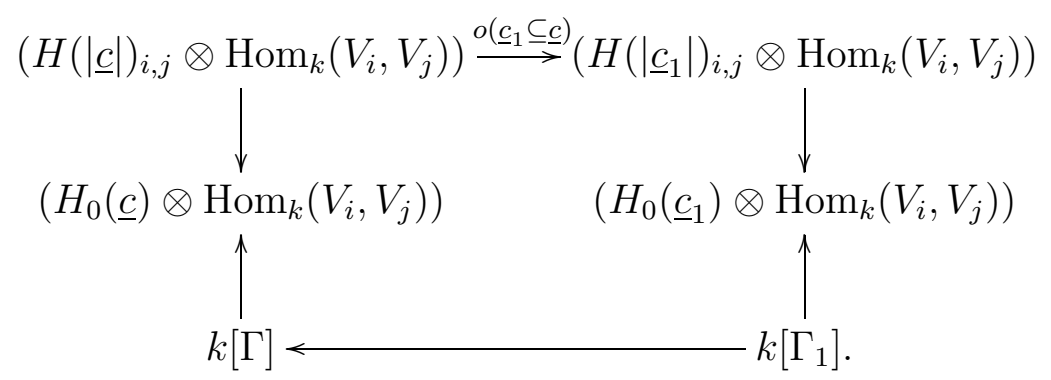

We would now like to conclude that this induces a natural morphism

$$
O\left(\underline{c}_{1} \subseteq \underline{c}\right): O(\underline{c}, \pi) \longrightarrow O\left(\underline{c}_{1}, \pi\right)
$$

since there are fewer conditions to be satisfied in the definition of the right hand algebra. However, the diagram above shows that this is not obvious. An element in $O(\underline{c}, \pi)$ certainly commutes with the action of $k\left[\Gamma_{1}\right]$ in $\left(H_{0}(\underline{c}) \otimes \operatorname{Hom}_{k}\left(V_{i}, V_{j}\right)\right)$ but not necessarily with the action of $k\left[\Gamma_{1}\right]$ in $\left(H_{0}\left(\underline{c}_{1}\right) \otimes \operatorname{Hom}_{k}\left(V_{i}, V_{j}\right)\right)$. But we are interested in the smallest $k$-algebra $O$ extending $A$, and preserving both the diagram and the system of iterated extensions of the objects of the diagram. Therefore we define the refined $k$-algebra of observables of the swarm $\underline{c}$, as,

Definition 3.14 The $k$-algebra of observables of the finite swarm $\underline{c}$, is the subalgebra

$$
\mathcal{O}(\underline{c}, \pi)=\bigcap_{\underline{c}_{0} \subseteq \underline{c}} o\left(\underline{c}_{0} \subseteq \underline{c}\right)^{-1}\left(O\left(\underline{c}_{0}, \pi\right)\right) \subseteq O(\underline{c}, \pi),
$$

where $\underline{c}_{0}$ runs through all subdiagrams of $\underline{c}$.

Now there is a natural homomorphism,

$$
\mathcal{O}\left(\underline{c}_{1} \subseteq \underline{c}\right): \mathcal{O}(\underline{c}, \pi) \rightarrow \mathcal{O}\left(\underline{c}_{1}, \pi\right),
$$

and it is easy to see that the $k$-algebra of observables is a contravariant functor on the ordered set of subdiagrams of a given finite diagram. 
We are now able to extend the definition of observables to certain infinite swarms.

Definition 3.15 A swarm is called permissible, if there exist a $k$-algebra homomorphism,

$$
\eta(|\underline{c}|, \pi): A \longrightarrow O(|\underline{c}|, \pi)
$$

compatible with the morphisms $\eta\left(\left|\underline{c}_{0}\right|, \pi\right)$ and $o\left(\underline{c}_{0} \subseteq \underline{c}\right)$, where $\underline{c}_{0}$ runs through all subdiagrams of $\underline{c}$.

For any permissible swarm $\underline{c}$, we define,

$$
\mathcal{O}(\underline{c}, \pi)=\lim _{\underline{c}_{0} \subseteq \underline{c}} \mathcal{O}\left(\underline{c}_{0}, \pi\right)
$$

where $\underline{c}_{0}$ runs through all finite subdiagrams of $\underline{c}$.

Clearly

$$
\mathcal{O}(\underline{c}, \pi) \subseteq O(|\underline{c}|, \pi)
$$

and $\eta(|\underline{c}|, \pi)$ induces a natural homomorphism of $k$-algebras,

$$
\eta(\underline{c}, \pi): A \longrightarrow \mathcal{O}(\underline{c}, \pi)
$$

the obvious limit of the family of morphisms $\eta\left(\underline{c}_{0}, \pi\right)$, where $\underline{c}_{0}$ runs through all finite subdiagrams of $\underline{c}$.

Definition 3.16 The permissible swarm $\underline{c}$ will be called a prescheme for $A$, if $\eta(\underline{c}, \pi)$ is an isomorphism.

Notice that if the finite swarm $\underline{c}$ is a prescheme in the sense of (2.8) then it is also a prescheme in the sense of (3.16). Therefore, any finite swarm $\underline{c}$ of $A$-modules is necessarily a prescheme for $O(\underline{c}, \pi)$. This is, however, not the case for infinite swarms, see Warning above.

\section{Noncommutative schemes}

Finally, we arrive at the notion of structure sheaf $\mathcal{O}_{\pi}$. For every finite subdiagram $\underline{c}_{0}$ of the swarm $\underline{c}$, consider the natural morphism,

$$
\kappa\left(\underline{c}_{0}\right): \mathcal{O}(\underline{c}, \pi) \rightarrow\left(H\left(\underline{c}_{0}\right) \otimes \operatorname{Hom}_{k}\left(V_{i}, V_{j}\right)\right)
$$

and consider the two-sided ideal $\mathfrak{n} \subset \mathcal{O}(\underline{c}, \pi)$, defined by

$$
\mathfrak{n}=\bigcap_{\underline{c}_{0} \subseteq \underline{c}} \operatorname{ker} \kappa\left(\underline{c}_{0}\right) .
$$

Here $\underline{c}_{0}$ runs through all finite subdiagrams of $\underline{c}$. 
Definition 3.17 (i) In the above situation, put,

$$
\mathcal{O}_{\pi}(\underline{c})=\mathcal{O}(\underline{c}, \pi) / \mathfrak{n}
$$

(ii) $\mathcal{O}_{\pi}$ is a presheaf on the ordered set of subdiagrams of a given permissible swarm $\underline{c}$.

Definition 3.18 (i) Let $A$ be any $k$-algebra. A permissible swarm $\underline{c}$ of $A$-modules is called a scheme for $A$, if the canonical morphism

$$
\eta(c, \pi): A \longrightarrow \mathcal{O}_{\pi}(\underline{c})
$$

is an isomorphism. In this case we shall call $A$ the affine $k$-algebra of $\underline{c}$.

(ii) We shall consider the objects $V_{i}$ of $\underline{c}$ as points, and the morphisms as incidences in our geometry.

(iii) Let for a point $V_{i}$ of $\underline{c}, H_{0}\left(V_{i}\right)$ be the modular substratum of the local moduli $H\left(V_{i}\right)$ of $V_{i}$, as $A$-module. There exists a natural morphism,

$$
\mathcal{O}_{\pi}(\underline{c}) \rightarrow H_{0}\left(V_{i}\right) \otimes \operatorname{End}_{k}\left(V_{i}\right)=\mathcal{O}_{\pi}\left(V_{i}\right) .
$$

When $\underline{c}$ is a scheme we shall refer to $O_{\pi}\left(V_{i}\right)$ as the local $\operatorname{ring}$ of $\underline{c}$ at $V_{i}$.

This is in tune with the general setup, see the Introduction. In fact, according to the Introduction, a space $\underline{c}$ should be the moduli space of its points, subject only to the conditions imposed by the incidences, i.e. by the morphisms of $\underline{c}$, and this is exactly the property of a scheme $\underline{c}$. The $k$-algebra $A=\mathcal{O}_{\pi}(\underline{c})$ is the universal $k$-algebra parametrizing the (modular) deformations of its points, subject only to the conditions imposed by the incidences.

Note that if a diagram $\underline{c}$ of $A$-modules is a prescheme for $A$, then it is not necessarily a scheme for $A$. However, we have the following obvious,

Lemma 3.19 (i) The diagram,

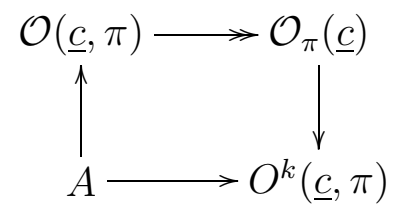

commutes.

(ii) If $\underline{c}$ is a prescheme for $A$ and the homomorphism $A \rightarrow O_{0}(\underline{c}, \pi)=$ $O^{k}(\underline{c}, \pi)$ is an isomorphism, then $\underline{c}$ is a scheme for $A$. 
Now, by (3.17) (ii), there exists for every finite family of objects $\left\{V_{l}\right\}_{l}$ of $\underline{c}$, considered as a discrete subdiagram of $\underline{c}$, a natural morphism,

$$
\mathcal{O}_{\pi}(\underline{c}) \rightarrow \mathcal{O}_{\pi}\left(\left\{V_{l}\right\}_{l}\right)
$$

If all $V_{l}$ 's are simple $A$-modules, we find that $H_{0}\left(V_{l}\right)=H\left(V_{l}\right)$, and,

$$
\mathcal{O}_{\pi}\left(\left\{V_{l}\right\}_{l}\right)=\left(H\left(\left\{V_{l}\right\}_{l}\right)_{i, j} \otimes \operatorname{Hom}_{k}\left(V_{i}, V_{j}\right)\right)
$$

We shall refer to $\mathcal{O}_{\pi}\left(\left\{V_{l}\right\}_{l}\right)$ as the semilocal ring of $\underline{c}$ at the family $\left\{V_{l}\right\}_{l}$.

Consider for any finite type $k$-algebra $A$, the discrete diagram, $\operatorname{Simp}(A)$, consisting of all the simple $A$-modules. If $A$ is finite dimensional, we know that the morphism,

$$
\eta(\operatorname{Simp}(A)): A \longrightarrow \mathcal{O}(\operatorname{Simp}(A), \pi)
$$

is an isomorphism provided $k$ is algebraically closed. In general $\eta(\operatorname{Simp}(A))$ is, however, far from an isomorphism. In fact, when $A$ is commutative, it is easy to see that

$$
\mathcal{O}(\operatorname{Simp}(A), \pi) \simeq \prod_{\mathfrak{m}} \hat{A}_{\mathfrak{m}}
$$

where $\mathfrak{m}$ runs through all maximal ideals of $A$. To obtain a result analogous to the General Burnside Theorem, see $\S 2$, we must add one or more generic points to $\operatorname{Simp}(A)$, obtaining a diagram $\operatorname{Simp}^{*}(A)$ consisting of all morphisms between the new generic $A$-modules and the simple ones. If we add just the $A$-module $A$, as we have explained above, we may now prove the following simple result,

Proposition 3.20 Suppose the natural homomorphism,

$$
\eta(\operatorname{Simp}(A)): A \longrightarrow \mathcal{O}(\operatorname{Simp}(A), \pi)
$$

is injective, then

$$
\eta\left(\operatorname{Simp}^{*}(A)\right): A \longrightarrow \mathcal{O}\left(\operatorname{Simp}^{*}(A), \pi\right)=\mathcal{O}_{\pi}\left(\operatorname{Simp}^{*}(A)\right)
$$

is an isomorphism, i.e. $\operatorname{Simp}^{*}(A)$ is a scheme for $A$.

Proof: Let us first prove this in the easiest case possible, i.e. where $A=\operatorname{End}_{k}(V)$, with $V$ a finite dimensional $k$-vector space of dimension $n$. Clearly there are $n$ linearly independent right $A$-module homomorphisms $\operatorname{pr}_{i}: A \rightarrow V, i=1, \ldots, n$. If $\left\{v_{i}\right\}$ is a basis for $V$ then for $a \in A, \operatorname{pr}_{i}(a)=v_{i} a=\sum_{j} h_{i, j}(a) v_{j}, h_{i, j}(a) \in k$. Moreover the morphism $\eta:=\eta(\operatorname{Simp}(A)): A \rightarrow O(\operatorname{Simp}(A), \pi)=\operatorname{End}_{k}(V)$, is an isomorphism and 
is given by $\eta(a)=\left(h_{i, j}(a)\right)$. Now, if $\alpha \in \operatorname{End}_{k}(A)$ sit in $\mathcal{O}\left(\operatorname{Simp}^{*}(A), \pi\right)$ then $\operatorname{pr}_{i}(\alpha(a))=\alpha_{0}\left(\operatorname{pr}_{i}(a)\right)$ for $\alpha_{0} \in \operatorname{End}_{k}(V)$ and for all $i=1, \ldots, n$. This means that $\eta(\alpha(a))=\alpha_{0}(\eta(a))$, therefore that $\eta(\alpha(1))=\alpha_{0}$, and therefore $\eta(\alpha(a)-a \alpha(1))=0$, so by injectivity of $\eta, \alpha(a)=a \alpha(1)$, for all $a \in A$. But this is exactly what we want, $\alpha$ is the right multiplication by some element $\alpha(1)$.

Now assume there are two simple modules, $V_{1}, V_{2}$, of $k$-dimensions, $n_{1}, n_{2}$. Then there are $n_{1} n_{2}$ morphisms in $\operatorname{Simp}^{*}(A)$, inducing the $A$-module homomorphisms, of $A$ into the versal family,

$$
p r_{r, s}: A \rightarrow\left(\begin{array}{ll}
H_{1,1} \otimes V_{1} & H_{1,2} \otimes V_{2} \\
H_{2,1} \otimes V_{1} & H_{2,2} \otimes V_{2}
\end{array}\right) .
$$

If $\left\{u_{r}\right\}$ and $\left\{v_{s}\right\}$ are bases of $V_{1}$ and $V_{2}$, we find as above,

$$
p r_{r, s}(a)=\left(\begin{array}{cc}
\sum_{k} h_{r, k}^{1,1}(a) \otimes u_{k} & \sum_{k} h_{r, k}^{1,2}(a) \otimes v_{k} \\
\sum_{l} h_{s, l}^{2,1}(a) \otimes u_{l} & \sum_{l} h_{s, l}^{2,2}(a) \otimes v_{l}
\end{array}\right)
$$

so, in particular,

$$
\eta(a)=\left(\begin{array}{ll}
\left(h_{i, j}^{1,1}(a)\right) & \left(h_{i, s}^{1,2}(a)\right) \\
\left(h_{r, j}^{2,1}(a)\right) & \left(h_{r, s}^{2,2}(a)\right)
\end{array}\right) .
$$

Now, as above, the condition on $\alpha \in \operatorname{End}_{k}(A)$ to sit in $\mathcal{O}\left(\operatorname{Simp}^{*}(A), \pi\right)$ is that there exist some $\alpha_{0} \in \mathcal{O}\left(\operatorname{Simp}^{*}(A), \pi\right)$ such that for all $a \in A, \eta(\alpha(a))=$ $\alpha_{0}(\eta(a))$. Therefore $\eta(\alpha(1))=\alpha_{0}$, and therefore $\eta(\alpha(a)-a \alpha(1))=0$, so by injectivity of $\eta, \alpha(a)=a \alpha(1)$, for all $a \in A$, such that $\alpha$ is the right multiplication by the element $\alpha(1) \in A$. This proof obviously generalizes to the case of any finite or infinite set of simples $\left\{V_{i}\right\}$.

For $A=k<x_{1}, x_{2}, \ldots, x_{d}>$, the free $k$-algebra on $d$ symbols, the example (2.9) shows that the condition of (3.20) is satisfied, therefore $\operatorname{Simp}_{n}^{*}(A)$, for any $n \geq 2$ is a scheme for $A$.

Notice that, so far we have defined a presheaf of observables on the family of subdiagrams of a permissible diagram $\underline{c}$ of $A$-modules. Starting with the geometry, i.e. the collection of points and incidences, we have defined, and studied, the algebra $\mathcal{O}_{\pi}(\underline{c})$ of operators parametrizing the geometry. When $\underline{c}$ is finite, the fact that $O(-, \pi)$ is a closure operator implies that the pair $(O:=O(\underline{c}, \pi), \underline{c})$ is a prescheme, such that $\eta: O \rightarrow O^{O}(\underline{c}, \pi)$ is an isomorphism. If also $\eta_{0}: O \rightarrow\left(H\left(\underline{c}_{0}\right) \otimes \operatorname{Hom}_{k}\left(V_{i}, V_{j}\right)\right)$ is an injection, it follows from $(3.17)$ that $(O, \underline{c})$ is a scheme. Now, if we start with a $k$-algebra $A$, how do we find its scheme? As we have seen in (3.11), there is no such thing, in this field, as a unique scheme associated to a given $k$-algebra. However as we have shown above, $\operatorname{Simp}^{*}(A)$ is a scheme in some interesting cases. Consequently, we are interested in the geometry of $\operatorname{Simp}(A)$. 
The structure of $\operatorname{Simp}_{n}(A)$

Lemma 3.21 If the $k$-algebra $A$ is finitely generated, then $\operatorname{Simp}_{<\infty}(A)$ is a swarm.

Proof: If $V_{i} \in \operatorname{Simp}_{m}(A)$ and $V_{j} \in \operatorname{Simp}_{n}(A)$, then $\operatorname{dim}_{k} \operatorname{Ext}_{A}^{1}\left(V_{i}, V_{j}\right)<\infty$. This follows from,

$$
\operatorname{Ext}_{A}^{1}\left(V_{i}, V_{j}\right)=\operatorname{Der}_{k}\left(A, \operatorname{Hom}_{k}\left(V_{i}, V_{j}\right)\right) / \text { Triv }
$$

since $A$ is generated by a finite number of generators as $k$-algebra. The rest follows from the next lemma, or more clearly from the proof of (3.23).

Lemma 3.22 Let $A$ be a finitely generated $k$-algebra and let $V \in \operatorname{Simp}_{n}(A)$, then the natural morphism,

$$
\eta(V): A \rightarrow H(V) \otimes_{k} \operatorname{End}_{k}(V),
$$

is topologically surjective.

Proof: We must prove that for any $m \geq 1$ the map,

$$
\eta_{m}: A \rightarrow H_{m} \otimes_{k} \operatorname{End}_{k}(V)
$$

is surjective, where $H_{m}=H(V) / \operatorname{Rad}(H(V))^{m}$. But this is obviously a consequence of the surjectivity of $\eta_{2}$. Now, $\eta_{1}: A \rightarrow \operatorname{End}_{k}(V)$ is surjective, by simplicity of $V$. Put $\mathfrak{m}=\operatorname{ker} \eta_{1}$, and consider the $A$-bi-module $\mathfrak{m} / \mathfrak{m}^{2}$. It is clearly an $\operatorname{End}_{k}(V)$-bimodule, and as such a finite sum of $r$ copies of $\operatorname{End}_{k}(V)$. Therefore $\operatorname{Der}_{k}\left(A, \operatorname{End}_{k}(V)\right)$, which is equal to the $k$-vector space of $k$-algebra homomorphisms, $\operatorname{Mor}_{k}\left(A, k[\epsilon] \otimes_{k} \operatorname{End}_{k}(V)\right)$ is identified with,

$$
\operatorname{Der}_{k}\left(\operatorname{End}_{k}(V), \operatorname{End}_{k}(V)\right) \oplus \operatorname{Hom}_{A \otimes A^{o p}}\left(\mathfrak{m} / \mathfrak{m}^{2},(\epsilon) \operatorname{End}_{k}(V)\right) .
$$

This, however is easily seen to be isomorphic to $\simeq \operatorname{Der}_{k}\left(\operatorname{End}_{k}(V)\right) \oplus k^{r}$. Since the derivations of $\operatorname{End}_{k}(V)$ are all inner, we find,

$$
\operatorname{Ext}_{A}^{1}(V, V) \simeq k^{r}
$$

But then, by construction, $H_{2} \simeq k\left[k^{r}\right]$, where $k\left[k^{r}\right]$ is the Nagata $k$-algebra of the vector space $k^{r}$, and the morphism $\eta_{2}$ is the obvious surjection, of $A$ onto $A / \mathfrak{m}^{2} \simeq k\left[k^{r}\right] \otimes_{k} \operatorname{End}_{k}(V)$

Another proof of a slightly more general result, is based on the generalized Burnside theorem, see $\S 2$. 
Lemma 3.23 Let $\mathcal{V}=\left\{V_{i}\right\}_{i=1, \ldots, r}$ be a finite subset of $\operatorname{Simp}_{<\infty}(A)$, then the morphism of $k$-algebras,

$$
A \rightarrow O(\mathcal{V})=\left(H_{i, j} \otimes_{k} \operatorname{Hom}_{k}\left(V_{i}, V_{j}\right)\right)
$$

is topologically surjective.

Proof: Since the simple modules $V_{i}, i=1, \ldots, r$ are distinct, there is an obvious surjection, $\pi: A \rightarrow \prod_{i=1, \ldots, r} \operatorname{End}_{k}\left(V_{i}\right)$. Put $\mathfrak{r}=\operatorname{ker} \pi$, and consider for $m \geq 2$ the finite-dimensional $k$-algebra, $B:=A / \mathfrak{r}^{m}$. Clearly $\operatorname{Simp}(B)=\mathcal{V}$, so that by the generalized Burnside theorem, see $\S 2$, we find, $B \simeq O^{B}(\mathcal{V}):=\left(H_{i, j}^{B} \otimes_{k} \operatorname{Hom}_{k}\left(V_{i}, V_{j}\right)\right)$. Consider the commutative diagram,

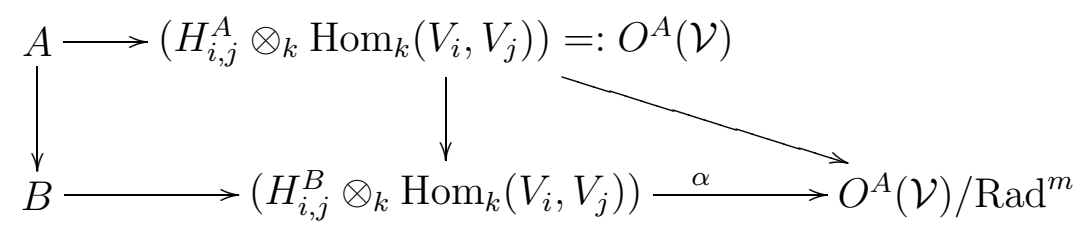

where all morphisms are natural. In particular $\alpha$ exists since $B=A / \mathfrak{r}^{m}$ maps into $O^{A}(\mathcal{V}) / \operatorname{Rad}^{m}$, and therefore induces the morphism $\alpha$ commuting with the rest of the morphisms. Consequently $\alpha$ has to be surjective, and we have proved the contention.

Recall that a standard $n$-commutator relation in a $k$-algebra $A$ is a relation of the type,

$$
\left[a_{1}, a_{2}, \ldots, a_{2 n}\right]:=\sum_{\sigma \in \Sigma_{2 n}} \operatorname{sign}(\sigma) a_{\sigma(1)} a_{\sigma(2)} \ldots a_{\sigma(2 n)}=0
$$

where $\left\{a_{1}, a_{2}, \ldots, a_{2 n}\right\}$ is a subset of $A$. Let $I(n)$ be the two-sided ideal of $A$ generated by the subset,

$$
\left\{\left[a_{1}, a_{2}, \ldots, a_{2 n}\right] \mid\left\{a_{1}, a_{2}, \ldots, a_{2 n}\right\} \subset A\right\} .
$$

Consider the canonical homomorphism,

$$
p_{n}: A \longrightarrow A / I(n)=: A(n) .
$$

It is well known that any homomorphism of $k$-algebras,

$$
\rho: A \longrightarrow \operatorname{End}_{k}\left(k^{n}\right)=: M_{n}(k)
$$

factors through $p_{n}$, see [4]. 
Corollary 3.24 (i) Let $m \geq 2$, then for any $V_{i}, V_{j} \in \operatorname{Simp}_{\leq n}(A)$ we have,

$$
\operatorname{Ext}_{A}^{1}\left(V_{i}, V_{j}\right) \simeq \operatorname{Ext}_{A / \mathfrak{r}^{m}}^{1}\left(V_{i}, V_{j}\right)
$$

(ii) Let $r=1$ above, and $V:=V_{1} \in \operatorname{Simp}_{n}(A)$. Then $I(n) \subset \mathfrak{r}^{2}$, and therefore,

$$
\operatorname{Ext}_{A}^{1}(V, V) \simeq \operatorname{Ext}_{A(n)}^{1}(V, V)
$$

Example 3.25 Notice that, for distinct $V_{i}, V_{j} \in \operatorname{Simp}_{\leq n}(A)$, we may well have,

$$
\operatorname{Ext}_{A}^{1}\left(V_{i}, V_{j}\right) \neq \operatorname{Ext}_{A(n)}^{1}\left(V_{i}, V_{j}\right) .
$$

In fact, consider the matrix $k$-algebra,

$$
A=\left(\begin{array}{cc}
k[x] & k[x] \\
0 & k[x]
\end{array}\right),
$$

and let $n=1$. Then $A(1)=k[x] \oplus k[x]$. Put $V_{i}=k[x] /(x) \oplus(0), V_{j}=$ $(0) \oplus k[x] /(x)$, then it is easy to see that,

$$
\operatorname{Ext}_{A}^{1}\left(V_{i}, V_{j}\right)=k, \operatorname{Ext}_{A(1)}^{1}\left(V_{i}, V_{j}\right)=0 .
$$

Lemma 3.26 Let $B$ be a k-algebra, and let $V$ a vector space of dimension $n$, such that the $k$-algebra $B \otimes \operatorname{End}_{k}(V)$ satisfies the standard $n$-commutatorcondition, i.e. such that the ideal, $I_{n} \subset B \otimes \operatorname{End}_{k}(V)$ generated by the standard commutators $\left[x_{1}, x_{2}, \ldots, x_{2 n}\right], x_{i} \in B \otimes \operatorname{End}_{k}(V)$ is zero. Then $B$ is commutative.

Proof: In fact if $b_{1}, b_{2} \in B$ is such that $\left[b_{1}, b_{2}\right] \neq 0$, then the obvious $n$-commutator,

$$
b_{1} e_{1,1} b_{2} e_{1,1} e_{1,2} e_{2,2} \ldots e_{n-1, n}-b_{2} e_{1,1} b_{1} e_{1,1} e_{1,2} e_{2,2} \ldots e_{n-1, n}
$$

is different from 0 .

Lemma 3.27 If $A$ is a finite type $k$-algebra, then any $V \in \operatorname{Simp}_{n}(A)$ is an $A(n):=A / I_{n}$-module, and the corresponding formal moduli, $H^{A(n)}(V)$ is isomorphic to $H^{A}(V)^{\mathrm{com}}$, the commutativization of $H^{A}(V)$.

Proof: Consider the natural diagram of homomorphisms of $k$-algebras,

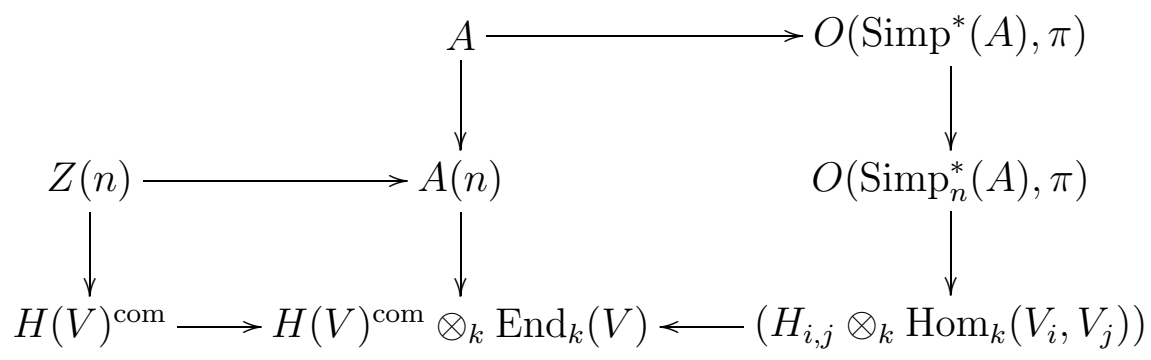


where $Z(n)$ is the center of $A(n):=A / I_{n}, V_{i}, V_{j} \in \operatorname{Simp}_{n}(A)$, and $H(V)^{\text {com }}$ is the commutativization of $H(V)$. Clearly there are natural morphisms of formal moduli,

$$
H^{A}(V) \rightarrow H^{A(n)}(V) \rightarrow H^{A}(V)^{\mathrm{com}} \rightarrow H^{A(n)}(V)^{\mathrm{com}} .
$$

Since moreover

$$
A(n) \rightarrow H^{A(n)}(V) \otimes \operatorname{End}_{k}(V)
$$

is topologically surjective, we find using (Lemma 3.26), that $H^{A(n)}(V)$ is commutative. But then the composition,

$$
H^{A(n)}(V) \rightarrow H^{A}(V)^{\mathrm{com}} \rightarrow H^{A(n)}(V)^{\mathrm{com}}
$$

is an isomorphism. Since by Corollary (3.24), the tangent spaces of $H^{A(n)}(V)$ and $H^{A}(V)$ are isomorphic, the lemma is proved.

Corollary 3.28 Let $A=k<x_{1}, \ldots, x_{d}>$ be the free $k$-algebra on $d$ symbols, and let $V \in \operatorname{Simp}_{n}(A)$. Then

$$
H^{A}(V)^{\mathrm{com}} \simeq H^{A(n)}(V) \simeq k\left[\left[t_{1}, \ldots, t_{(d-1) n^{2}+1}\right]\right]
$$

This should be compared with the results of Procesi, see [14], or [4]. There are further examples, some based upon the calculation of Tord Romstad, see [15], showing that $H^{A}(V)$ is not commutative, even though $V \in$ $\operatorname{Simp}(A)=\operatorname{Simp}_{\leq 2}(A)$.

In general we do not know that the natural morphism,

$$
A(n) \rightarrow \prod_{V \in \operatorname{Simp}_{n}(A)} H^{A(n)}(V) \otimes_{k} \operatorname{End}_{k}(V)
$$

is an injection. Replace $A$ by its quotient, $O(n):=O_{\pi}\left(\operatorname{Simp}_{n}(A(n))\right.$, then for all $V \in \operatorname{Simp}_{n}(A)$,

$$
H^{O}(V) \simeq H^{A(n)}(V)
$$

and,

$$
O(n) \rightarrow \prod_{V \in \operatorname{Simp}_{n}(A)} H^{O(n)}(V) \otimes_{k} \operatorname{End}_{k}(V)
$$

is injective. Put $B=\prod_{V \in \operatorname{Simp}_{n}(A)} H^{A(n)}(V)$. Let $x_{i} \in A, i=1, \ldots, d$ be generators of $A$, and consider the images $\left(x_{p, q}^{i}\right) \in B \otimes_{k} \operatorname{End}_{k}\left(k^{n}\right)$ of $x_{i}$ via the injective homomorphism of $k$-algebras,

$$
O(n) \rightarrow B \otimes \operatorname{End}_{k}\left(k^{n}\right),
$$


obtained by choosing bases in all $V \in \operatorname{Simp}_{n}(A)$. Now, $B$ is commutative, so the $k$-subalgebra $C \subset B$ generated by the elements $\left\{x_{p, q}^{i}\right\}_{i=1, \ldots, d ; p, q=1, \ldots, n}$ is commutative. We have an injection,

$$
O(n) \rightarrow C \otimes_{k} \operatorname{End}_{k}\left(k^{n}\right)
$$

and for all $V \in \operatorname{Simp}_{n}(A)$ there is a natural projection,

$$
C \otimes_{k} \operatorname{End}_{k}\left(k^{n}\right) \rightarrow H^{A(n)}(V) \otimes_{k} \operatorname{End}_{k}(V) .
$$

Since $A(n) \rightarrow H^{A(n)}(V) \otimes_{k} \operatorname{End}_{k}(V)$ is topologically surjective, $H^{A(n)}(V) \otimes_{k}$ $\operatorname{End}_{k}(V)$ is generated by the images of $x_{i}$. It follows that, if $t(V)$ is the element of $\operatorname{Simp}(C)$ defined by $V$, we have a surjective homomorphism,

$$
\hat{C}_{t(V)} \rightarrow H^{A(n)}(V)
$$

Categorical properties imply, as usual, that there is a natural morphism,

$$
H^{A(n)}(V) \rightarrow \hat{C}_{t(V)}
$$

which composed with the former is an automorphism of $H^{A(n)}(V)$. It follows that $H^{A(n)}(V)$ is a projection of the commutative $k$-algebra $\hat{C}_{t(V)}$. Clearly $\operatorname{Simp}_{n}(A) \subset \operatorname{Simp}(C)$. Let $C(n)$ be the affine algebra of the closure of $\operatorname{Simp}_{n}(A)$ in $\operatorname{Simp}(C)$. Then it is clear that for every $V \in \operatorname{Simp}_{n}(A)$ there is an isomorphism $H^{A(n)}(V) \simeq \hat{C}_{t(V)}(n)$, and so also,

$$
O(n) \subset C(n) \otimes_{k} \operatorname{End}_{k}\left(k^{n}\right) .
$$

Recall the commutative diagram,

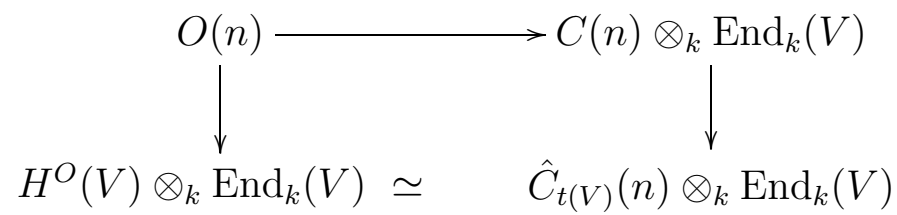

Using Nakayama's lemma, it is easy to see that locally, at a point $V \in$ $\operatorname{Simp}_{n}(A), C(n)$ and $Z(O(n))$ are isomorphic, which implies a result of M. Artin, see [1], stating that if $A$ does not have any simple modules of dimension less than $n$, then $A(n)$ is an Azumaya algebra over its center $Z(n)$.

Moreover, $\operatorname{Simp}(C(n))$ is, in a sense, a compactification of $\operatorname{Simp}_{n}(A)$, and we shall be able, using this imbedding to study the degeneration processes that occur, at infinity in $\operatorname{Simp}_{n}(A)$, see a forthcoming paper. 
Now, consider for $s_{2} \leq s_{1} \leq n, V_{1} \in \operatorname{Simp}_{s_{1}}(A), V_{2} \in \operatorname{Simp}_{s_{2}}(A)$, the commutative diagram,

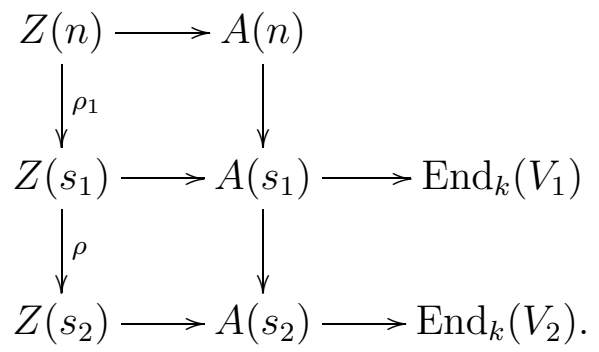

Put $\rho_{2}:=\rho \rho_{1}$, and let $t\left(V_{i}\right) \in \operatorname{Simp}\left(Z\left(s_{i}\right)\right)$ be the points corresponding to the simple modules $V_{i}$.

Lemma 3.29 In the situation above, if $\operatorname{Ext}_{A(n)}^{1}\left(V_{i}, V_{j}\right) \neq 0$ then

$$
\rho_{i}: \operatorname{Simp}\left(Z\left(s_{i}\right)\right) \rightarrow \operatorname{Simp}(Z(n)), i=1,2,
$$

maps $t\left(V_{1}\right)$ and $t\left(V_{2}\right)$ to the same point.

Proof: If $\rho_{1}\left(t\left(V_{1}\right)\right) \neq \rho_{2}\left(t\left(V_{2}\right)\right)$, the two corresponding maximal ideals $\mathfrak{m}_{i}, i=1,2$, of $Z(n)$ will be distinct, the sum $\mathfrak{m}_{1}+\mathfrak{m}_{2}$ is then $Z(n)$. However, $\mathfrak{m}_{i}$ annihilates $V_{i}$, therefore the sum will annihilate $\operatorname{Ext}_{A}^{1}\left(V_{i}, V_{j}\right)$, which therefore must be zero.

\section{Localization and topology on $\operatorname{Simp}(A)$}

Let $s \in A$, and consider the open subset $D(s)$ of $\operatorname{Simp}(A)$, defined above. It is clear that the natural morphism,

$$
\eta: A \rightarrow \mathcal{O}_{\pi}(D(s))
$$

maps $s$ into an invertible element of $\mathcal{O}_{\pi}(D(s))$. Therefore we may define the localization $A_{\{s\}}$ of $A$, as the $k$-algebra $\mathcal{O}_{\pi}(D(s))$ with the inverse $\eta(s)$ added. This furnishes a general method of localization with all the properties one would wish.

\section{Generic points}

In the general case, given a diagram $\underline{c}$, we should be prepared to include in $\underline{c}$ a finite subset $\gamma(\underline{c})$ of generic points, and modify the construction of $\mathcal{O}_{\pi}(\underline{c})$, such that the result,

$$
\mathcal{O}_{\pi}(\underline{c}, \gamma(\underline{c}))
$$

parametrizes the deformations of all points of $\underline{c}$, subject to the conditions imposed by the incidences and, moreover, keeping the generic points fixed. 
This construction also turns out to give us the right tool to define a reasonable notion of noncommutative subscheme.

To obtain $\mathcal{O}_{\pi}(\underline{c}, \gamma(\underline{c}))$ we copy the construction of $\mathcal{O}_{\pi}(\underline{c})$, replacing $O(|\underline{c}|, \pi)$ by the subring

$$
O(|\underline{c}|, \gamma(\underline{c}) ; \pi)=\left(H(|\underline{c}|, \gamma(\underline{c}))_{i, j} \otimes \operatorname{Hom}_{k}\left(V_{i}, V_{j}\right)\right)
$$

where,

$$
\begin{array}{ll}
H(|\underline{c}|, \gamma(\underline{c}))_{i, j}=H(|\underline{c}|)_{i, j} & \text { if } V_{i} \notin \gamma(\underline{c}) \\
H(|\underline{c}|, \gamma(\underline{c}))_{i, j}=0 & \text { if } i \neq j, V_{i} \in \gamma(\underline{c}) \\
H(|\underline{c}|, \gamma(\underline{c}))_{i, i}=k & \text { if } V_{i} \in \gamma(\underline{c}),
\end{array}
$$

and, for every finite subdiagram $\underline{c}_{0}$ of $\underline{c}$, while insisting that $\underline{c}_{0}$ contain $\gamma(\underline{c})$, by replacing $H\left(\underline{c}_{0}\right)$ by $H\left(\underline{c}_{0}, \gamma(\underline{c})\right)$ given by the cocartesian diagram,

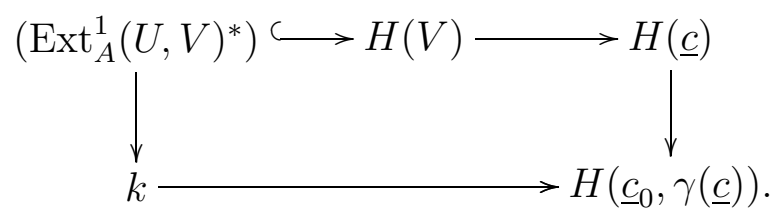

Here,

$$
U=\oplus_{V_{i} \in \gamma(\underline{c})} V_{i} \subseteq V=\oplus_{V_{i} \in|\underline{c}|} V_{i}
$$

and $\left(\operatorname{Ext}_{A}^{1}(U, V)^{*}\right)$ is the ideal of $H(V)$ generated by a dual basis of $\operatorname{Ext}_{A}^{1}(U, V)$, considered as part of a dual basis of $\operatorname{Ext}_{A}^{1}(V, V)$ generating $H(V)$.

Examples 3.30 (i) Let $A$ be any $k$-algebra, and let $\underline{c}:=\operatorname{Ind}(A)$ be the diagram consisting of the essential (i.e. generating all others) morphisms between (all) the indecomposable $A$-modules. Suppose $A$ is a sum of a finite number of indecomposibles. Using the fact that the only $k$-linear endomorphisms of $A$ that are right $A$-linear, are the left multiplication by elements of $A$, we easily prove that $\eta_{0}: A \rightarrow O_{0}(\operatorname{Ind}(A), \pi)$ is an isomorphism. Since $\operatorname{Ind}(A)$ is, essentially, a finite diagram, we may use the closure property of $\mathcal{O}$ and prove that $\operatorname{Ind}(A)$ is a prescheme for $A$ and therefore, by (3.18), also a scheme.

(ii) Consider a discrete diagram $\underline{c}=\mathcal{V}$ of $A$-modules. There is, by definition of $\mathcal{O}_{\pi}$, a homomorphism,

$$
\mathcal{O}_{\pi}(\mathcal{V}) \longrightarrow\left(H_{0}(V) \otimes \operatorname{Hom}_{k}\left(V_{i}, V_{j}\right)\right)
$$

The tangent space of the image is $\left(E_{i, j} \otimes \operatorname{Hom}_{k}\left(V_{i}, V_{j}\right)\right)$, where the $E_{i, j}$ are defined by,

$$
\left(E_{i, j}\right)=\left(\operatorname{Ext}_{A}^{1}\left(V_{i}, V_{j}\right)\right)^{\operatorname{End}_{A}(V)}
$$


(iii) In particular, if $\mathcal{V}$ is the family of all simple right modules of an artinian $k$-algebra $A$, and $k$ is algebraically closed, then $\operatorname{End}_{A}(V)$ is trivial, and we obtain,

$$
A \simeq \mathcal{O}_{\pi}(\mathcal{V})
$$

so $\mathcal{V}$ is a scheme for $A$.

(iv) Going back to Example (3.13), where $A$ was a local $k$-algebra with $\phi: A \rightarrow k$ as the residue map, we found that when $\phi_{*}: \operatorname{Ext}_{A}^{1}(k, A) \rightarrow$ $\operatorname{Ext}_{A}^{1}(k, k)$ is zero, the natural morphism,

$$
\eta: A \rightarrow \mathcal{O}(\{\phi\}, \pi)
$$

is an isomorphism. It is now clear that, in all cases,

$$
\eta: A \rightarrow \mathcal{O}_{\pi}(\{\phi\})
$$

is an isomorphism, implying that $\{\phi\}$ is a scheme for $A$.

(v) The Hairy Line. Consider the $k$-algebra of matrices,

$$
A=\left(\begin{array}{cc}
k[y] & k[y] \\
0 & k[y]
\end{array}\right)
$$

The scheme $\operatorname{Ind}(A)$ contains the two projectives,

$$
V_{1,2}=\left(\begin{array}{cc}
k[y] & k[y] \\
0 & 0
\end{array}\right), V_{2}=\left(\begin{array}{cc}
0 & 0 \\
0 & k[y]
\end{array}\right)
$$

such that $A=V_{1,2} \oplus V_{2}$. Therefore $\operatorname{Ind}(A)$ is a scheme for $A$. Notice that there is an incidence, i.e. a morphism of (right) $A$-modules $V_{2} \rightarrow V_{1,2}$, the cokernel of which is the indecomposible $A$-module,

$$
V_{1}=\left(\begin{array}{cc}
k[y] & 0 \\
0 & 0
\end{array}\right) \text {. }
$$

Notice also that $A_{i}:=V_{i}, i=1,2$ are quotient algebras of $A$ both isomorphic to the polynomial $k$-algebra $k[y]$. The closed points of $\operatorname{Ind}(A)$ are the different simple representations of $A$, that is, the different closed points of $\operatorname{Spec}\left(A_{i}\right)$, for $i=1,2$. Thus we find that the closed points correspond to the points of two different affine lines, $L_{1}$ and $L_{2}$, both (canonically) isomorphic to $\operatorname{Spec}(k[y])$. However, while there are no ordinary incidences between these points, there are infinitesimal incidences between pairs of equal points $(p, p) \in L_{1} \times L_{2}$. In fact if $p \in L_{1}, q \in L_{2}$, then

$$
\begin{aligned}
& \operatorname{Ext}_{A}^{1}(k(p), k(p))=k \\
& \operatorname{Ext}_{A}^{1}(k(q), k(q))=k \\
& \operatorname{Ext}_{A}^{1}(k(p), k(q))=k \text { if } p=q \\
& \operatorname{Ext}_{A}^{1}(k(p), k(q))=0 \text { if } p \neq q \\
& \operatorname{Ext}_{A}^{1}(k(q), k(p))=0 \text { for all } p \in L_{1}, q \in L_{2} .
\end{aligned}
$$


The picture of this is is an ordinary line $L_{2}$ with hairs, corresponding to the points of the other line $L_{1}$, stuck into the first line at the corresponding point.

Let as above, $\operatorname{Simp}(A)$ be the discrete diagram of the simple $A$-modules, and let $\operatorname{Simp}^{*}(A)$ be the diagram consisting of $A$ and $\operatorname{Simp}(A)$ together with the obvious morphisms. We claim that $\operatorname{Simp}^{*}(A)$ is a scheme for $A$. In fact this follows from the following calculations: Let $p=q$, and let $\xi_{1,1} \in \operatorname{Ext}_{A}^{1}(k(p), k(p)), \xi_{2,2} \in \operatorname{Ext}_{A}^{1}(k(q), k(q))$ and $\xi_{1,2} \in \operatorname{Ext}_{A}^{1}(k(p), k(q))$, be generators. Then the cup products $\xi_{1,1} \cup \xi_{1,2} \in \operatorname{Ext}_{A}^{2}(k(p), k(q))$ and $\xi_{1,2} \cup$ $\xi_{2,2} \in \operatorname{Ext}_{A}^{2}(k(p), k(q))$ are equal and different from 0 . Since $\operatorname{Hom}_{k}(k, k)=k$ this implies,

$$
\mathcal{O}_{\pi}(\{k(p), k(q)\})=H(\{k(p), k(q)\})=\left(\begin{array}{cc}
k[[y]] & k[[y]] \\
0 & k[[y]]
\end{array}\right) .
$$

and the natural morphism

$$
\eta: A \rightarrow \mathcal{O}_{\pi}(\{k(p), k(q)\})
$$

is the obvious completion. From this follows immediately,

$$
\mathcal{O}_{\pi}\left(\operatorname{Simp}^{*}(A)\right)=\left(\begin{array}{cc}
k[y] & k[y] \\
0 & k[y]
\end{array}\right)
$$

\section{Noncommutative subschemes}

Let $\left(\underline{c}_{0}, \gamma\left(\underline{c}_{0}\right)\right)$ be a swarm of $A$-modules and its subset of generic points. Suppose first that $\gamma\left(\underline{c}_{0}\right)=\left\{V_{0}\right\}$, i.e. is reduced to one single object. Let $\rho$ : $V_{0} \rightarrow V_{1}$ be any homomorphism of $A$-modules. Consider now the morphisms $\phi: V_{1} \rightarrow V_{p}$ of $\underline{C}=A$-mod, for which the composition $\rho \phi$ is a morphism of $\underline{c}_{0}$. The resulting diagram $\underline{c}_{1}$ with generic point $\left.\gamma\left(\underline{c}_{1}\right)\right)=\left\{V_{1}\right\}$ should be considered as a closed subobject of $\left(\underline{c}_{0}, \gamma\left(\underline{c}_{0}\right)\right)$ cut out by $\rho$. Notice that $\underline{c}_{1}$ deprived of its generic point, is a subdiagram of $\underline{c}_{0}$. It is clear how to generalize this notion, but we shall leave that for later work. Consider the following example.

Example 3.31 (i) The quantum plane. Let $A=k_{q}[x, y]:=k\{x, y\} /(x y-$ $q y x), k$ algebraically closed, with $q \neq 1$. The discrete diagram $\operatorname{Simp}(A)$ may be identified with the union of the $x$ - and the $y$-axes in the affine plane. Consider points $p_{1}, p_{2} \in \operatorname{Simp}(A)$, then we easily compute,

$$
\operatorname{Ext}_{A}^{1}\left(k\left(p_{1}\right), k\left(p_{2}\right)\right)=k,
$$


if $p_{1}=p_{2} \neq 0$ or if $p_{1}$ and $p_{2}$ sit on the $x$-axis and $q p_{2}=p_{1}$.

$$
\operatorname{Ext}_{A}^{1}\left(k\left(p_{1}\right), k\left(p_{2}\right)\right)=k
$$

if $p_{1}$ and $p_{2}$ sit on the $y$-axis and $q p_{1}=p_{2}$. In particular,

$$
\begin{aligned}
& \operatorname{Ext}_{A}^{1}(k(0), k(0))=k^{2} \\
& \operatorname{Ext}_{A}^{2}(k(0), k(0))=k .
\end{aligned}
$$

All other Ext's are 0, and there exists a basis $\left\{\xi_{1}, \xi_{2}\right\}$ of $\operatorname{Ext}_{A}^{1}(k(0), k(0))$, such that

$$
0 \neq \xi_{1} \cup \xi_{2}=q \xi_{2} \cup \xi_{1} \in \operatorname{Ext}_{A}^{2}(k(0), k(0))
$$

Therefore,

$$
H^{A}(k(0)) \simeq \hat{A}_{(0)},
$$

and so $A \simeq \mathcal{O}_{\pi}\left(\operatorname{Simp}^{*}(A)\right)$. Moreover, if $V_{1}=A /(a x+b y+c)$ is a line in the quantum plane, and if we consider the corresponding closed subobject (Simp* $\left.(A),\left\{V_{1}\right\}\right)$ of $\operatorname{Simp}^{*}(A)$, in the manner described above, we obtain, $\mathcal{O}_{\pi}\left(\operatorname{Simp}^{*}(A),\left\{V_{1}\right\}\right) \simeq A /(a x+b y+c)$, as we would expect.

(ii) Espaces quantiques de Connes, trivial case. Let $A$ be a commutative $k$-algebra, $k$ algebraically closed. Consider an algebraic equivalence relation, $\underline{R}=\operatorname{Spec}(R)$ on the affine scheme $X=\operatorname{Spec}(A)$. It corresponds to the affine diagram,

$$
A \underset{i_{2}}{\stackrel{i_{1}}{\longrightarrow}} A \otimes A \stackrel{\rho}{\longrightarrow} R \longrightarrow A
$$

with the obvious relations. Consider the morphisms $\tau_{i}=i_{i} \circ \rho$, and let

$$
\operatorname{Spec}(A: R)=\left\{R / \tau_{1}(\mathfrak{p}) R \mid \mathfrak{p} \in \operatorname{Spec}(A)\right\}
$$

be the diagram consisting of the objects $R / \mathfrak{p} R:=R / \tau_{1}(\mathfrak{p}) R$ considered as $A$-modules via $\tau_{2}$, and with the obvious morphisms. Let $x \in X$ be a closed point, corresponding to the maximal ideal $\underline{m}_{x}$ of $A$, then the composition,

$$
\kappa_{x}: A \stackrel{i_{2} \circ \rho}{\longrightarrow} R \stackrel{\pi_{x}}{\longrightarrow} R / \underline{m}_{x} R
$$

identifies $\operatorname{Spec}\left(R / \underline{m}_{x} R\right)$ with the equivalence class $\bar{x}$ of $x \in X=\operatorname{Spec}(A)$. In particular, if $x \sim_{R} y$ then

$$
R / \underline{m}_{x} R=R / \underline{m}_{y} R
$$

as objects of $\operatorname{Spec}(A: R)$. Now compute

$$
O_{0}:=O_{0}(\operatorname{Spec}(A: R), \pi) \text {. }
$$

It is clear that $\alpha \in O_{0}$ is a family

$$
\alpha=\left\{\alpha_{\mathfrak{p}} \in \operatorname{End}_{k}\left(R / \tau_{1}(\mathfrak{p}) R\right) \mid \pi_{\mathfrak{q}}^{\mathfrak{p}} \circ \alpha_{\mathfrak{q}}=\alpha_{\mathfrak{p}} \circ \pi_{\mathfrak{q}}^{\mathfrak{p}}, \forall \mathfrak{p}, \mathfrak{q} \in \operatorname{Spec}(A)\right\}
$$


where $\pi_{\mathfrak{q}}^{\mathfrak{p}}: R / \tau_{1}(\mathfrak{p}) R \rightarrow R / \tau_{1}(\mathfrak{q}) R$ is the obvious morphism corresponding to an inclusion $\mathfrak{p} \subseteq \mathfrak{q}$. Since each one of the $\pi_{\mathfrak{q}}^{\mathfrak{p}}$ is surjective, it is easy to see that $\alpha$ is determined by $\alpha_{0} \in \operatorname{End}_{k}(R)$ and the components $\alpha_{x} \in \operatorname{End}_{k}\left(R / \underline{m}_{x} R\right)$. Moreover, since for $x \sim y, R / \underline{m}_{x} R=R / \underline{m}_{y} R$, as objects, it is clear that $\alpha_{x}$ depends only upon the equivalence class $\bar{x}$ of $x$. Each $\alpha_{\bar{x}}$ is a $k$-linear endomorphism. Suppose the equivalence classes of $R$ are finite reduced, i.e. $R / \underline{m}_{x} R \simeq \oplus_{z \in \bar{x}} k(z)$, then $\alpha_{\bar{x}}$ is a matrix

$$
\alpha_{\bar{x}}=\left(\alpha\left(z, z^{\prime}\right)\right), \quad z, z^{\prime} \in \bar{x}, \alpha\left(z, z^{\prime}\right) \in k .
$$

Moreover, in this case, given $\alpha, \alpha^{\prime} \in O_{0}$ then $\alpha=\alpha^{\prime}$ if and only if $\alpha_{\bar{x}}=\alpha_{\bar{x}}^{\prime}$ for all closed points $x \in X$. This follows immediately from the defining relations, $\pi_{x}(\alpha(a))=\alpha_{\bar{x}}\left(\pi_{x}(a)\right)$. This means that $\alpha$ is determined as a function on $X \times X$ with $\alpha\left(z, z^{\prime}\right)=0$ unless $z \sim z^{\prime}$, and with addition defined as for functions, but with multiplication defined by the matrix nature of each $\alpha_{\bar{x}}$, i.e. $\left(\alpha \alpha^{\prime}\right)(x, y)=\sum_{z \in \bar{x}=\bar{y}} \alpha(x, z) \alpha^{\prime}(z, y)$. But this is the way Connes defines les espaces quantiques, see [3].

\section{Infinitesimal structures on schemes}

Let $\underline{c}$ be a swarm of $A$-mod, and consider a point $x=V_{i}$. We would like to be able to talk about vector fields, their values at points, if possible also about energy operators and time etc. as in quantum mechanics. We start with the following,

Definition 3.32 Given a point $x=V_{i} \in \underline{c}$, we put

$$
\begin{aligned}
T_{x}:= & \operatorname{Ext}_{A}^{1}\left(V_{i}, V_{i}\right)^{\Gamma}: \\
= & \left\{\xi \in \operatorname{Ext}_{A}^{1}\left(V_{i}, V_{i}\right) \mid \forall p \exists \xi_{p} \in \operatorname{Ext}_{A}^{1}\left(V_{p}, V_{p}\right) \text { such that } \forall \phi:=\phi_{i, p}: V_{i} \rightarrow V_{p},\right. \\
& \left.\phi_{*}(\xi)=\phi^{*}\left(\xi_{p}\right) \text { and } \forall \phi:=\phi_{p, i}: V_{p} \rightarrow V_{i}, \phi_{*}\left(\xi_{p}\right)=\phi^{*}(\xi)\right\}
\end{aligned}
$$

and we shall call it the tangent space of $\underline{c}$ at $x$.

There is a canonical map

$$
\kappa_{x}: \operatorname{Der}_{k}(A, A) \longrightarrow T_{x}
$$

the compositions of the natural maps,

$$
\operatorname{Der}_{k}(A, A) \longrightarrow \operatorname{Der}_{k}\left(A, \operatorname{End}_{k}(V)\right)
$$

the surjection

$$
\operatorname{Der}_{k}\left(A, \operatorname{End}_{k}(V)\right) \longrightarrow \operatorname{Ext}_{A}^{1}(V, V)=\oplus_{i, j} \operatorname{Ext}_{A}^{1}\left(V_{i}, V_{j}\right)
$$

and the projection onto $\operatorname{Ext}_{A}^{1}\left(V_{i}, V_{i}\right)$. 
Recall that the tangent space $T_{0}$ of $H(\underline{c})$ is contained in,

$$
T_{0}=\left(\operatorname{Ext}_{A}^{1}(V, V)^{\Gamma}\right)^{\operatorname{End}_{k[\Gamma] \otimes A}(V)}
$$

and see that there are natural homomorphisms $\operatorname{Der}_{k}(A, A) \rightarrow T_{0}$.

For every $\delta \in \operatorname{Der}_{k}(A, A)$, let $\delta(x) \in T_{x}$, be the image $\kappa_{x}(\delta)$ in $T_{x}$. Thus $\operatorname{Der}_{k}(A, A)$ is a $k$-vector space of vector fields defined on $\underline{c}$. In particular if $E \in A$ is some element, then the $k$-linear map adE $: A \rightarrow A$ defined by $a d E(a)=E a-a E$, is a $k$-derivation of $A$. Obviously, $a d E(x)=0$ for every point $x$ of $\underline{c}$. Thus, operators of this type (like time evolutions), i.e. $\operatorname{ad}(E)$, although they act on the observables, leave the points fixed.

Definition 3.33 Given a point $\mathrm{x}=V_{i}$ of $\underline{c}$, we shall say that $x$ is $A$-smooth if the map $\kappa_{x}$ is surjective. If this is true for all points of $\underline{c}$, we shall say that $\underline{c}$ is $A$-smooth.

Using the example (2.9) we observe that when $A$ is a free $k$-algebra, the scheme $\operatorname{Simp}^{*}(A)$ is smooth for $A$, and it clearly generalizes the classical notion of smoothness in algebraic geometry.

There is also the non-commutative version of Grothendieck's notion of smoothness, the Quillen, or Q-smoothness. The $k$-algebra $A$ is Q-smooth if whenever $R \rightarrow S$ is a surjective small homomorphism of $k$-algebras, the map $\operatorname{Mor}(A, R) \rightarrow \operatorname{Mor}(A, S)$ is surjective. The following result is easily proved.

Proposition $3.34 A$ is Q-smooth implies that for all finite dimensional simple A-module $V$, all cup and Massey products,

$$
\operatorname{Ext}_{A}^{1}(V, V) \otimes \operatorname{Ext}_{A}^{1}(V, V) \otimes \cdots \otimes \operatorname{Ext}_{A}^{1}(V, V) \rightarrow \operatorname{Ext}_{A}^{2}(V, V)
$$

vanish.

From this we deduce the well known result,

Corollary 3.35 If a finite type commutative $k$-algebra $A$ is $Q$-smooth then either $A \simeq k^{n}$ for some $n$, or $A$ is a smooth curve.

\section{The commutative case}

\section{The main Theorem}

To show that the noncommutative algebraic geometry, introduced above, is a good extension of classical algebraic geometry, one would be tempted to prove that, for commutative $k$-algebras $A$,

$$
\eta(\operatorname{Spec}(A), \pi): A \longrightarrow \mathcal{O}_{\pi}(\operatorname{Spec}(A))
$$

is an isomorphism. 
This is, however, not reasonable, see the Introduction. The problem is that $\operatorname{Spec}(A)$ contains too many points. The closed points are special since $\operatorname{Spec}(A)$ is the moduli space for such. The non closed points are not treated as bona fide points, but as generic points for subschemes. We would like to include as few as possible such generic points, and therefore we add only the projective generator $A$. We have already proved the essentials of the following,

Theorem 4.1 Let $A$ be any commutative $k$-algebra, essentially of finite type, with $k$ algebraically closed. Let, as above $\operatorname{Simp}^{*}(A)$ be the diagram $\operatorname{Simp}(A)$ augmented by the generic point $A$. Then $\operatorname{Simp}^{*}(A)$ is a permissible swarm, and the canonical morphism of k-algebras,

$$
\eta\left(\operatorname{Simp}^{*}(A), \pi\right): A \rightarrow \mathcal{O}_{\pi}\left(\operatorname{Simp}^{*}(A)\right)
$$

is an isomorphism.

Proof: For every closed point $x \in \operatorname{Simp}(A)$ consider the corresponding homomorphisms of $A$-modules,

$$
\phi_{x}: A \rightarrow k(x)
$$

and use the Remark (3.6) together with (3.20). Clearly the versal deformation of $\phi_{x}$ is the canonical morphism of $k$-algebras

$$
\Phi_{x}: A \rightarrow H(k(x)) \otimes \operatorname{End}_{k}(k(x))=\hat{A}_{x} .
$$

If $\alpha \in \operatorname{End}_{k}(A)$ and $\alpha_{x} \in \operatorname{End}_{H_{x, x}}\left(H(k(x)) \otimes \operatorname{End}_{k}(k(x))\right)$ commute via the action of $\Phi_{x}$, then the composition $\left(\alpha-R_{\alpha(1)}\right) \circ \Phi_{x}=0$, where $R_{\alpha(1)}$ is the right multiplication by $\alpha(1)$. Since this is true for all $x \in \operatorname{Simp}(A)$ we obtain that $\alpha$ is the right multiplication of some element $\alpha(1)$, proving our theorem.

As a consequence of the functorial properties of $\mathcal{O}_{\pi}$ the $\mathcal{O}_{\pi}$-construction applies to the (open affine) subdiagrams of the category of $O_{X}$-Mod, where $X$ is a $k$-scheme, and provides us with a globalization procedure. To see how it works, let us consider the following example.

Example 4.2 Blowing-ups. Let $A=k[x, y]$ and consider the $A$-module $V=(x, y)$, i.e. the maximal ideal of $A$. The diagram of $A$-modules, $\operatorname{Simp}^{*}(A-V)$ is, by definition, the diagram consisting of $V$ together with all simple quotients of $V$. These correspond to all points of $\mathbf{A}^{2}=\operatorname{Spec}(A)$ different from $(0,0)$, together with all tangent lines through $(0,0)$ in $\mathbf{A}^{2}$. Now consider $x \in V$ and let $D(x)$ be the diagram obtained from $\operatorname{Simp}^{*}(A-V)$ by 
localizing, i.e. removing the points where $x$ becomes zero, and inverting the multiplicative action by $x$. In particular, in $D(x)$ we have all points of $\mathbf{A}^{2}$ minus the $y$-axis, but preserving all tangent lines through $(0,0)$, except the $x$-axis. We find,

$$
\mathcal{O}\left(\operatorname{Simp}^{*}(A-V), \pi\right)=A, \mathcal{O}(D(x), \pi)=k[y, y / x]
$$

as we should, proving that the scheme $\left(\operatorname{Simp}^{*}(A-V), \mathcal{O}_{\pi}\right)$ is the blow up of the origin in $\mathbf{A}^{2}$.

Notice that in this paragraph we have assumed that $A$ is a commutative $k$-algebra essentially of finite type on an algebraically closed field. The extension of the theory to include schemes on general base rings, seems difficult.

\section{More homological preparations}

\section{The category of $A-G$-modules}

Let $A$ be any $k$-algebra and let $g: A \rightarrow A$ be an automorphism. Given an $A$ module $M_{i}, i=1,2$, consider an automorphism of $k$-modules $\nabla_{g}^{i}: M_{i} \rightarrow M_{i}$, such that for $m_{i} \in M_{i}$ and $a \in A$ we have,

$$
\nabla_{g}^{i}\left(m_{i} a\right)=\nabla_{g}^{i}\left(m_{i}\right) g(a) \text { for } i=1,2
$$

i.e. such that $\nabla_{g}^{i}$ is $g$-linear. Then there is an automorphism,

$$
\theta_{g}^{p}:=\theta_{g}^{p}\left(\nabla^{1}, \nabla^{2}\right): \operatorname{Ext}_{A}^{p}\left(M_{1}, M_{2}\right) \longrightarrow \operatorname{Ext}_{A}^{p}\left(M_{1}, M_{2}\right)
$$

induced via the isomorphism,

$$
\operatorname{Ext}_{A}^{p}\left(M_{1}, M_{2}\right) \simeq H H^{p}\left(A, \operatorname{Hom}_{k}\left(M_{1}, M_{2}\right)\right)
$$

by the $g^{-1}$-linear automorphism of bi-modules,

$$
\zeta_{g}: \operatorname{Hom}_{k}\left(M_{1}, M_{2}\right) \longrightarrow \operatorname{Hom}_{k}\left(M_{1}, M_{2}\right)
$$

defined by,

$$
\psi \longmapsto \nabla_{g}^{1} \circ \psi \circ \nabla_{g^{-1}}^{2} .
$$

Notice that we compose morphisms in the natural order. For $a \in A$ we compute,

$$
\begin{aligned}
& \zeta_{g}(g(a) \psi)=\nabla_{g}^{1} \circ g(a) \psi \circ \nabla_{g^{-1}}^{2}=a\left(\nabla_{g}^{1} \circ \psi \circ \nabla_{g^{-1}}^{2}\right)=a \zeta_{g}(\psi) \\
& \zeta_{g}(\psi g(a))=\nabla_{g}^{1} \circ \psi g(a) \circ \nabla_{g^{-1}}^{2}=\left(\nabla_{g}^{1} \circ \psi \circ \nabla_{g^{-1}}^{2}\right) a=\zeta_{g}(\psi) a .
\end{aligned}
$$


This implies that there is an automorphism of Hochschild cohomology,

$$
\zeta_{g}^{p}: H H^{p}\left(A, \operatorname{Hom}_{k}\left(M_{1}, M_{2}\right)\right) \longrightarrow H H^{p}\left(A, \operatorname{Hom}_{k}\left(M_{1}, M_{2}\right)\right)
$$

defined on cochain form by,

$$
\xi^{p} \longmapsto\left\{\left(a_{1}, a_{2}, \ldots, a_{p}\right) \mapsto \nabla_{g}^{1} \circ \xi^{p}\left(g\left(a_{1}\right), \ldots, g\left(a_{p}\right)\right) \circ \nabla_{g^{-1}}^{2}\right\} .
$$

In particular the automorphism,

$$
\zeta_{g}^{1}: \operatorname{Ext}_{A}^{1}\left(M_{1}, M_{2}\right) \longrightarrow \operatorname{Ext}_{A}^{1}\left(M_{1}, M_{2}\right)
$$

is induced by the map

$$
\zeta_{g}^{1}: \operatorname{Der}_{k}\left(A, \operatorname{Hom}_{k}\left(M_{1}, M_{2}\right)\right) \longrightarrow \operatorname{Der}_{k}\left(A, \operatorname{Hom}_{k}\left(M_{1}, M_{2}\right)\right)
$$

defined by

$$
\zeta_{g}^{1}(\delta)(a)=\nabla_{g}^{1} \circ \delta(g(a)) \circ \nabla_{g^{-1}}^{2}
$$

When $\mathfrak{p} \subseteq A$ is a $g$-invariant ideal of $A$ contained in the annihilator of $M_{2}$, we know that the restriction of the derivations of $\operatorname{Der}_{k}\left(A, \operatorname{Hom}_{k}\left(M_{1}, M_{2}\right)\right)$ to $\mathfrak{p}$ induces an isomorphism,

$$
\operatorname{Hom}_{A}\left(\mathfrak{p} / \mathfrak{p}^{2}, \operatorname{Hom}_{A}\left(A / \mathfrak{p}, M_{2}\right)\right) \simeq \operatorname{Ext}_{A}^{1}\left(A / \mathfrak{p}, M_{2}\right)
$$

such that the automorphism $\zeta_{g}^{1}$ takes the form,

$$
\zeta_{g}^{1}(\psi)(x)=\nabla_{g^{-1}}^{2}(\psi(g x)) \text { for } x \in \mathfrak{p} / \mathfrak{p}^{2} .
$$

Suppose $\xi \in \operatorname{Ext}_{A}^{1}\left(M_{1}, M_{2}\right)$ is represented by the exact sequence of $A$ modules,

$$
0 \longrightarrow M_{2} \longrightarrow E \longrightarrow M_{1} \longrightarrow 0
$$

Since the $g$-linear automorphisms $\nabla_{g}^{i}: M_{i} \rightarrow M_{i}$ correspond to an $A$-linear isomorphism,

$$
\nabla_{g}^{i}: M_{i} \rightarrow M_{i} \otimes_{g^{-1}} A
$$

we deduce from $(*)$ the exact sequence of $A$-modules,

$$
0 \longrightarrow M_{2} \otimes_{g^{-1}} A \longrightarrow E \otimes_{g^{-1}} A \longrightarrow M_{1} \otimes_{g^{-1}} A \longrightarrow 0
$$

which represents the element $\zeta_{g}^{1}(\xi) \in \operatorname{Ext}_{A}^{1}\left(M_{1}, M_{2}\right)$. The $\zeta_{g}^{1}$-invariant elements $\xi$ of $\operatorname{Ext}_{A}^{1}\left(M_{1}, M_{2}\right)$ therefore corresponds to the extensions $(*)$ for which there exists an isomorphism

$$
\nabla_{g}: E \longrightarrow E \otimes_{g^{-1}} A
$$

compatible with the $\nabla_{g}^{i}$, for $i=1,2$. Another way of viewing this is to look at $\zeta_{g}^{1}(\xi)-\xi$ as an obstruction for the existence of such an isomorphism $(* *)$. 
Given one $\nabla_{g}: E \longrightarrow E \otimes_{g^{-1}} A$ compatible with the $\nabla_{g}^{i}$, another $\nabla_{g}^{\prime}$ will differ from the first one by the composition $\Gamma_{g}$ of the homomorphism $E \longrightarrow M_{1}$ and some $A$-linear map $\alpha: M_{1} \rightarrow M_{2} \otimes_{g^{-1}} A$, and any such $\Gamma_{g}$ added to $(* * *)$, will again be compatible with the $\nabla_{g}^{i}$, for $i=1,2$. In the category of $(A-g)$-modules, we therefore find,

$$
\operatorname{Ext}_{A-g}^{1}\left(M_{1}, M_{2}\right) \simeq \operatorname{Ext}_{A}^{1}\left(M_{1}, M_{2}\right)^{\zeta_{g}} \oplus \operatorname{Hom}_{A}\left(M_{1}, M_{2} \otimes_{g^{-1}} A\right) / \sim
$$

The equivalence $\sim$ identifies $\left(E^{\prime}, \nabla_{g}^{\prime}\right)$ and $\left(E^{\prime \prime}, \nabla_{g}^{\prime \prime}\right)$ if there exists an isomorphism of extensions $\zeta: E \simeq E^{\prime \prime}$ compatible with the $\nabla$ 's. Since

$$
\nabla_{g}^{2}: \operatorname{Hom}_{A}\left(M_{1}, M_{2}\right) \simeq \operatorname{Hom}_{A}\left(M_{1}, M_{2} \otimes_{g^{-1}} A\right)
$$

the equivalence relation $\sim$ is trivial.

Now, suppose $G$ is a group acting on the $k$-algebra $A$, i.e. suppose there exists a homomorphism of groups,

$$
\rho: G \longrightarrow \operatorname{Aut}_{k}(A) \text {. }
$$

Consider $A$-modules $M_{i}, i=1,2$, with $G$-actions compatible with $\rho$, i.e. homomorphisms

$$
\nabla^{i}: G \longrightarrow \operatorname{Aut}_{k}\left(M_{i}\right)
$$

such that for $g \in G, m_{i} \in M_{i}$, and $a \in A$,

$$
\nabla_{g}^{i}\left(m_{i} a\right)=\nabla_{g}^{i}\left(m_{i}\right) g(a) \text { for } i=1,2
$$

where we denote by $g(a)$ the action of $\rho(g)$ on $a \in A$.

Given an invariant $\xi \in \operatorname{Ext}_{A}^{1}\left(M_{1}, M_{2}\right)$ under the action of the group $G$, as explained above, there exists for every $g \in G$ an isomorphism

$$
\nabla_{g}: E \longrightarrow E \otimes_{g^{-1}} A
$$

Since

$$
\left(E \otimes_{g_{1}^{-1}} A\right) \otimes_{g_{2}^{-1}} A=E \otimes_{\left(g_{1} g_{2}\right)^{-1}} A
$$

we find an obstruction for the existence of a homomorphism of groups,

$$
\nabla: G \longrightarrow \operatorname{Aut}_{k}(E)
$$

compatible with the $\nabla^{i}$ s, which is a 2-cocycle of $G$ with values in the $G$ bimodule $\operatorname{Hom}_{A}\left(M_{1}, M_{2}\right)$,

$$
\left(g_{1}, g_{2}\right) \longmapsto\left(\nabla_{g_{1}} \circ \nabla_{g_{2}}-\nabla_{g_{1} g_{2}}\right) .
$$

When the corresponding 2-class,

$$
\sigma_{\xi} \in H^{2}\left(G, \operatorname{Hom}_{A}\left(M_{1}, M_{2}\right)\right)
$$

vanishes, there exists a $\nabla$ and the set of such will be a torsor under

$$
H^{1}\left(G, \operatorname{Hom}_{A}\left(M_{1}, M_{2}\right)\right) \text {. }
$$


Proposition 5.1 Suppose $H^{i}\left(G, \operatorname{Hom}_{A}\left(M_{1}, M_{2}\right)\right)=0$ for $i=1,2$. Then,

$$
\operatorname{Ext}_{A-G}^{1}\left(M_{1}, M_{2}\right) \simeq \operatorname{Ext}_{A}^{1}\left(M_{1}, M_{2}\right)^{G} .
$$

Notice that a 1-coboundary of the form

$$
g \longmapsto(g \alpha-\alpha)
$$

corresponds to an automorphism $\theta_{\alpha}: E \longrightarrow E$ inducing an automorphism of $\left(E, \nabla_{g}\right)$.

\section{The category of $A-\mathfrak{g}$-modules}

Suppose

$$
\rho: \mathfrak{g} \longrightarrow \operatorname{Der}_{k}(A)
$$

is a $k$-Lie homomorphism, e.g. a Lie-Cartan pair. We shall treat this as the tangent map of a Lie-group action $\rho$ studied in the previous section. Let $M_{i}, i=1,2$ be $A$-modules with $\mathfrak{g}$-integrable connections

$$
\nabla^{i}: \mathfrak{g} \longrightarrow \operatorname{End}_{k}\left(M_{i}\right)
$$

and consider for every $\delta \in \mathfrak{g}$ and every $\psi \in \operatorname{Hom}_{k}\left(M_{1}, M_{2}\right)$ the map

$$
\delta \longmapsto \nabla_{\delta}^{1} \psi-\psi \nabla_{\delta}^{2}
$$

This defines a Lie algebra homomorphism,

$$
\rho: \mathfrak{g} \longrightarrow \operatorname{End}_{k}\left(\operatorname{Hom}_{k}\left(M_{1}, M_{2}\right)\right)
$$

such that, if $\rho$ is a Lie-Cartan pair, $\rho(\delta a)=a \rho(\delta)-\rho(\delta) a$.

Let $D \in \operatorname{Der}_{k}\left(A, \operatorname{Hom}_{k}\left(M_{1}, M_{2}\right)\right)$, then the map

$$
a \longmapsto \nabla_{\delta}(D)(a):=D(\delta(a))+\nabla_{\delta}^{1} D(a)-D(a) \nabla_{\delta}^{2}
$$

is a derivation, and we obtain a connection

$$
\nabla: \mathfrak{g} \longrightarrow \operatorname{End}_{k}\left(\operatorname{Ext}_{A}^{1}\left(M_{1}, M_{2}\right)\right) .
$$

As above, every $\xi \in \operatorname{Ext}_{A}^{1}\left(M_{1}, M_{2}\right)^{\mathfrak{g}}$ is associated to an obstruction,

$$
\sigma(\xi) \in H^{2}\left(\mathfrak{g}, \operatorname{Hom}_{k}\left(M_{1}, M_{2}\right)\right)
$$

which vanishes if and only if there exists an integrable connection on the middle term $E$ of the exact sequence representing $\xi$,

$$
0 \longrightarrow M_{2} \longrightarrow E \longrightarrow M_{1} \longrightarrow 0
$$

compatible with the connections $\nabla^{i}$ on $M_{i}$. The set of isomorphism classes of such $(\xi, \nabla)$ is then a torsor under

$$
H^{1}\left(\mathfrak{g}, \operatorname{Hom}_{A}\left(M_{1}, M_{2}\right)\right) .
$$

Proposition 5.2 Suppose $H^{i}\left(\mathfrak{g}, \operatorname{Hom}_{A}\left(M_{1}, M_{2}\right)\right)=0$ for $i=1,2$. Then,

$$
\operatorname{Ext}_{A-\mathfrak{g}}^{1}\left(M_{1}, M_{2}\right)=\operatorname{Ext}_{A}^{1}\left(M_{1}, M_{2}\right)^{\mathfrak{g}}
$$




\section{Invariant theory and moduli}

Let $k$ be an algebraically closed field, and consider a commutative finite type $k$-algebra $A$. Let $G$ be a finite group, acting on $A$, and let $\rho: G \rightarrow \operatorname{Aut}_{k}(A)$, be the action. Consider the category of $A-G$-modules, and let, as above, $\operatorname{Simp}^{*}(A-G)$ be the swarm of orbits of $G$ on $\operatorname{Spec}(A)$, together with $A$ as generic point. For any orbit $V \in \operatorname{Simp}(A-G)$, let $v \in \operatorname{Spec}\left(A^{G}\right)$ be the corresponding point. If $G$ is reductive we know that the ideal $\mathfrak{a} \subset A$ defining a maximal orbit $V$, a regular point, is generated by the ideal $\mathfrak{a}_{0}=\mathfrak{a}^{G} \subset A^{G}$. In fact, since the ideal $\mathfrak{a}$ is generated by invariants, the relation-submodule will also be generated by invariants, etc. Therefore there exist an $A-G$ resolution of $V$, of the form,

$$
0 \lessdot V \longleftarrow A \leftarrow A^{n_{1}} \longleftarrow A^{n-2} \ldots
$$

where the action by $G$ is the obvious product-action. Since $G$ is reductive we obtain the following resolution of the field $k=V^{G}=\operatorname{End}_{A-G}(V)$ as $A^{G}$-module,

$$
0<\quad k \longleftarrow \quad A^{G \leftarrow}\left(A^{G}\right)^{n_{1}} \longleftarrow\left(A^{G}\right)^{n-2} \ldots
$$

This implies that,

$$
\operatorname{Ext}_{A-G}^{p}(V, V)=\operatorname{Ext}_{A^{G}}^{p}(k, k),
$$

for all $p \geq 0$ and the Massey-product structures also coincide. Therefore, by construction $H(V) \simeq \hat{A}_{v}^{G}$. For all such maximal orbits $V$ we have a natural commutative diagram,

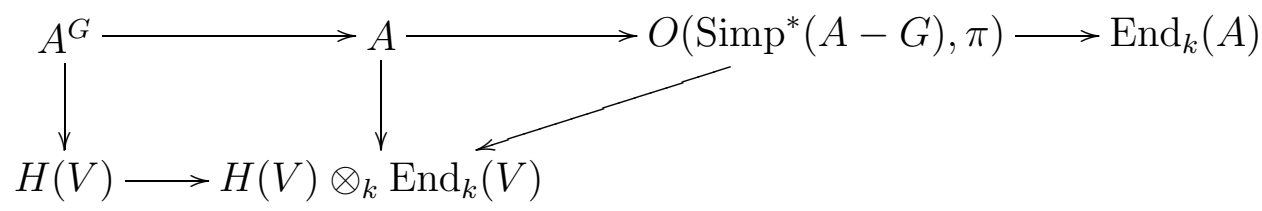

showing that,

$$
O\left(\operatorname{Simp}^{*}(A-G), \pi\right) \subset \operatorname{End}_{A^{G}}(A) .
$$

Let $I \subset G$, be a subgroup. Put $X(I) \subseteq\left\{x \in X:=\operatorname{Simp}(A) \mid I=g I(x) g^{-1}\right\}$ be the locally closed subscheme of $X:=\operatorname{Spec}(A)$, corresponding to the orbits with isotropy subgroups conjugate to $I$. Let $\mathfrak{a}(I) \subset A$ be the $G$-invariant ideal of $A$, defining the closure $\bar{X}(I)$ in $X$. Put $A(I):=A / \mathfrak{a}(I)$. Consider a simple $A-G$-module $V(I)$, with isotropy subgroup class $I$. Assume that the natural morphism of right $A-G$-module, $A \rightarrow H(V(I)) \otimes V(I)$, is the formalization of a morphism $A \rightarrow \mathcal{H}(V(I)) \otimes V(I)$ with $\mathcal{H}(V(I))$ a $k$-algebra of finite type. Notice that since $G$ is reductive, $G$ acts on $\mathcal{H}(V(I)) \otimes V(I)$ 
via its action on $V(I)$, i.e. the action lifts trivially to $\mathcal{H}(V(I)) \otimes V(I)$. Then there is a commutative diagram,

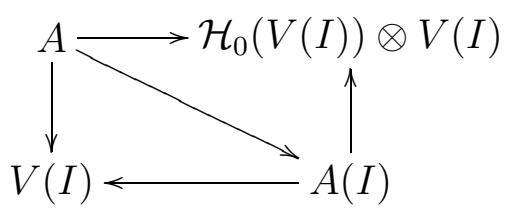

where $\mathcal{H}_{0}(V(I))=\mathcal{H}(V(I)) / \operatorname{Rad}$, is the reduced $k$-algebra. Here Rad is the intersection of all two-sided maximal ideals with residual field $k$. Let $H_{0}(V(I))=\hat{\mathcal{H}}_{0}(V(I))$, the formalization of $\mathcal{H}_{0}(V(I))$ at the point corresponding to $V(I)$. This diagram, however, proves that there are natural morphisms,

$$
H^{A(I)-G}(V(I)) \rightarrow H_{0}^{A-G}(V(I)) \rightarrow H_{0}^{A(I)-G}(V(I)) .
$$

If $V(I)$ is regular with respect to the $G$-action on $X(I)$, we therefore have,

$$
H_{0}^{A-G}(V(I)) \simeq(\widehat{A(I)})_{v \text { red }}^{G}
$$

In the example (8.3), below, in characteristic 2, Tord Romstad has computed the formal moduli $H^{A-G}(V(I))$ for $I=G$, and come up with a noncommutative $k$-algebra such that its reduction is isomorphic to

$$
H^{A(I)-G}(V(I)) \simeq k[[t]],
$$

see the end of $\S 8$, and [15].

Definition 6.1 The quotient $\operatorname{Simp}^{*}(A) / G$, is the swarm of $O\left(\operatorname{Simp}^{*}(A-\right.$ $G), \pi)$-modules $\operatorname{Simp}^{*}(A-G)$.

Proposition 6.2 $\operatorname{Simp}^{*}(A-G)$ is a scheme for $O:=\mathcal{O}\left(\operatorname{Simp}^{*}(A-G), \pi\right)$

Proof: This follows from (3.20) since, for each maximal orbit $V_{d}, A_{v_{d}}$ is a free $A_{v_{d}}^{G}$-module, and since the natural map $O \rightarrow O(\operatorname{Simp}(O), \pi)$ decomposes via $\operatorname{End}_{A^{G}}(A) \rightarrow \prod_{V_{d}} A_{v_{d}}^{G} \otimes \operatorname{End}_{k}\left(V_{d}\right)$ which, since the maximal orbits correspond to an open dense set in $\operatorname{Spec}\left(A^{G}\right)$, is injective.

Notice that in $\operatorname{Simp}^{*}(A-G)$ the point $A$ is generic. See also that there is a natural surjective homomorphism, $H^{O}(V) \rightarrow H^{A-G}$ which, since

$$
O \rightarrow \prod_{V} H^{A-G}(V) \otimes \operatorname{End}_{k}(V)
$$

is injective, makes

$$
O \rightarrow \prod_{V} H^{O}(V) \otimes \operatorname{End}_{k}(V)
$$

also injective. By (3.20) this shows that also $\operatorname{Simp}^{*}(O)$ is a scheme for $O$. Here $O$ is the generic point. 
Example 6.3 Let $G=\mathbb{Z} /(2)=(\tau)$ act on $A=k[x, y]$ as $\tau(x)=x, \tau(y)=$ $x-y$. Then $A^{G}=k\left[x, x y-y^{2}\right]=: k[u, v]$. Then $\operatorname{Simp}^{*}(A-G)$ is a scheme for the $k$-algebra,

$$
\left\{\left(\begin{array}{ll}
a_{1,1} & a_{1,2} \\
a_{2,1} & a_{2,2}
\end{array}\right) \mid a_{i, j} \in A^{G}, a_{1,1}-a_{2,2} \in\left(u^{2}-4 v\right), a_{1,2}, a_{2,1} \in\left(u^{2}-4 v\right)\right\}
$$

If $G$ is a Lie group acting algebraically on the $k$-algebra $A$, we may copy the definitions and the proofs above, and obtain the same results. However since in this case the orbits, i.e. the points of $\operatorname{Simp}(A-G)$, are of infinite $k$ dimension, we shall work with the trivialization functor, $\pi_{G}: A-G$-mod $\rightarrow$ $k$-mod defined by $\pi_{G}(V)=V^{G}=H^{0}(G, V)$. When $G$ is reductive consider the stratification $\{X(I)\}$ of $X=\operatorname{Simp}(A)$, indexed by all conjugacy classes of (isotropy) subgroups $I \subset G$. Consider the set of generic points $\{A(I)\}$, and let us construct $O:=O^{A-G}\left(\operatorname{Simp}^{*}(A-G) ;\{A(I)\}\right)$. G operates on $O$, and the invariant ring maps to the $k$-algebra $H^{A-G}(\operatorname{Simp}(A-G))=$ : $\left(H_{i, j}^{A-G}\right)$. Let $O^{A-G}\left(\operatorname{Simp}^{*}\left(A-G ;\{A(I)\}, \pi_{G}\right)\right.$ be the image.

Definition 6.1 bis. The quotient $\operatorname{Simp}^{*}(A) / G$, is the swarm $\operatorname{Simp}^{*}(O)$ of $O$-modules, where,

$$
O:=O^{A-G}\left(\operatorname{Simp}^{*}(A-G), \pi_{G}\right) .
$$

Notice that there is a natural identification $\operatorname{Simp}^{*}(A-G) \simeq \operatorname{Simp}^{*}(O)$, but $O$ is no longer an extension of $A$. However, we have the natural morphism $\eta_{G}: A^{G} \rightarrow O$. We shall return to the properties of this construction. See $\S 8$ for examples.

Now, let the Lie-algebra $\mathfrak{g}$ of vectorfields (i.e. derivations), act on $A$. Consider the category $\underline{C}:=A-\mathfrak{g}$-mod, of $A$-modules with integrable $\mathfrak{g}$-covariant derivations. In this category we define the trivialization functor,

$$
\pi_{\mathfrak{g}}: \underline{C} \longrightarrow k-\bmod
$$

by $\pi_{\mathfrak{g}}(V)=H^{0}(\mathfrak{g}, V)$.

Recall that when $\mathfrak{g}$ is reductive $\pi:=\pi_{\mathfrak{g}}$ is exact. Moreover, Ext in this category, is then simply the $\mathfrak{g}$-invariants of the Ext in $A$-mod.

Given any permissible swarm $\underline{c}$ of $\underline{C}$, we may construct a ring of preobservables $O^{A-\mathfrak{g}}\left(\underline{c}, \pi_{\mathfrak{g}}\right)$, in the same way as we constructed $O^{A-G}\left(\operatorname{Simp}^{*}(A-\right.$ $\left.G), \pi_{G}\right)$ in the case of a group action. In particular, let us consider the diagram, $\operatorname{Simp}(A-\mathfrak{g})$, of simple $A-\mathfrak{g}$-modules, and add to it $A$, as a generic point, to obtain $\operatorname{Simp}^{*}(A-\mathfrak{g})$. 
We propose, tentatively,

Definition 6.1 bis, bis. The quotient $\operatorname{Simp}^{*}(A) / \mathfrak{g}$, is the swarm $\operatorname{Simp}^{*}(O)$, of $O$-modules, where,

$$
O:=O^{A-\mathfrak{g}}\left(\operatorname{Simp}^{*}(A-\mathfrak{g}), \pi_{\mathfrak{g}}\right) .
$$

Notice that we do not assert that $\operatorname{Simp}^{*}(A-\mathfrak{g})$, as a diagram of $A-\mathfrak{g}$-mod, is a scheme for $O$. This would be meaningless. In good cases we may, however, hope that $\operatorname{Simp}^{*}(O)$ is a scheme for $O$, and that $\operatorname{Simp}(\mathcal{O})$ is, in a natural way, isomorphic to $\operatorname{Simp}(A-\mathfrak{g})$.

In many cases the simple $A-\mathfrak{g}$-modules occur in a finite number of families, just as in the case of a group action, corresponding to quotients of some $U_{l}=A / \mathfrak{a}_{l}$. In this case it is reasonable to add the $U_{l}$ 's as generic points to $\operatorname{Simp}(A-\mathfrak{g})$ forming a new diagram $\operatorname{Simp}^{*}(A-\mathfrak{g})$. We would then consider $\mathcal{O}_{\pi_{\mathfrak{g}}}\left(\operatorname{Simp}^{*}(A-\mathfrak{g}),\left\{U_{l}\right\}\right)$ as a candidate for the affine ring of the orbit space.

That this invariant theory fits with the classical invariant theory, is shown by the following result,

Theorem 6.4 Let $A$ be any irreducible and reduced commutative $k$-algebra of finite type, $k$ algebraically closed, and $\mathfrak{g}$ a reductive Lie-algebra of vectorfields (i.e. derivations), acting on A. Assume that the geometric quotient of $\operatorname{Spec}(A)$ with $\mathfrak{g}$ exists and is affine. Then

$$
O^{A-\mathfrak{g}}\left(\operatorname{Simp}^{*}(A-\mathfrak{g}), \pi_{\mathfrak{g}}\right)=A^{\mathfrak{g}} .
$$

Proof: By assumption, the diagram $\operatorname{Simp}^{*}\left(A^{\mathfrak{g}}\right)$ induces the diagram $\operatorname{Simp}^{*}(A-\mathfrak{g})$. Moreover the trivialization $\pi_{\mathfrak{g}}$ maps the diagram $\operatorname{Simp}^{*}(A-\mathfrak{g})$ onto the diagram $\operatorname{Simp}^{*}\left(A^{\mathfrak{g}}\right)$, or rather, to the image of this diagram under the canonical trivialization $\pi$. But then the exactness of $\pi_{\mathfrak{g}}$ and the smoothness of the morphism of the geometric quotient, proves that the formal moduli of the family $\left|\operatorname{Simp}^{*}(A-\mathfrak{g})\right|$ in the category of $A-\mathfrak{g}$-mod is isomorphic to the corresponding formal moduli of the family $\left|\operatorname{Simp}^{*}\left(A^{\mathfrak{g}}\right)\right|$ in the category of $A^{\mathfrak{g}}$-mod. Since the trivializations coincide, the Theorem (6.1) shows that

$$
O\left(\operatorname{Simp}^{*}(A-\mathfrak{g}), \pi_{\mathfrak{g}}\right) \cong A^{\mathfrak{g}}
$$

which is exactly what we wanted.

Example 6.5 (The McKay correspondence) Let $k$ be an algebraically closed field of characteristic 0 , and let $U$ be a finite dimensional $k$-vector space. Put $n=\operatorname{dim}_{k} U$. Let $G$ be any group acting on $U$, and let $\left\{V_{i}\right\}_{i=1, \ldots, r}$ be the family of simple $G$-modules, such that

$$
k(G)=\prod_{i=1, \ldots, r} V_{i}^{n_{i}}
$$

as $k(G)$-modules. 
Let $A=\operatorname{Sym}_{k}\left(U^{*}\right)$, and consider now a quotient module $o$ of $A$ which is isomorphic to $k(G)$ as $G$-module, i.e. an object of the diagram $\operatorname{Simp}^{\text {s.s }}(A-$ $G)$. There must exist a filtration of $o$ with graded module consisting of the different $V_{i}$ strung out in some order, defined by an ordered graph $\Gamma$ of length $r$. If $x \in \underline{o}$ is a closed point, and $I_{x}$ the corresponding isotropy subgroup of $G$, we know that $o$ has an $A-G$-quotient of the form,

$$
A / \mathfrak{m}=\prod_{g \in I_{x} / G} k(g(x))
$$

which is an object of $\operatorname{Simp}^{*}(A-G)$. In this way we find a map,

$$
\kappa: \operatorname{Simp}^{s . s}(A-G) \longrightarrow \operatorname{Simp}^{*}(A-G)
$$

which is the McKay map. There are interesting cases in which

$$
\mathcal{O}\left(-, \pi_{G}\right)
$$

is a non singular presheaf of commutative $k$-algebras on the (open) subdiagrams of $\operatorname{Simp}^{s . s}(A-G)$, with a canonical birational morphism,

$$
\kappa^{*}: \mathcal{O}\left(\operatorname{Simp}^{*}(A-G), \pi_{G}\right)=A^{G} \rightarrow \mathcal{O}\left(-, \pi_{G}\right)
$$

showing that $\kappa$ is a desingularization of $\operatorname{Spec}(A) / G$. Suppose $G$ acts effectively, such that $\operatorname{Simp}^{s . s}(A-G) \cap \operatorname{Simp}^{*}(A-G)$ is an open dense set of $\operatorname{Simp}^{*}(A-G) \subseteq \operatorname{Spec}\left(A^{G}\right)$, in the Zariski topology. Then $o$ is a non singular point of $\operatorname{Simp}^{s . s}(A-G)$, if and only if,

$$
\begin{aligned}
\chi(o, o): & =\sum_{i=1, \ldots, n}(-1)^{i} \operatorname{ext}_{A-G}^{i}(o, o) \\
& =\sum_{p=1, \ldots, n, i, j=1, \ldots, r}(-1)^{p} \operatorname{hom}_{G}\left(V_{i}, V_{j} \otimes \wedge^{p} U\right)=0 .
\end{aligned}
$$

Notice that for the scheme to be commutative we must have,

$$
\operatorname{ext}_{A-G}^{2}(o, o) \geq \operatorname{dim}_{k}\left(\operatorname{Ext}_{A-G}^{1}(o, o) \wedge \operatorname{Ext}_{A-G}^{1}(o, o)\right) \geq 1 / 2 n(n-1) .
$$

Moreover, we always have,

$$
\operatorname{hom}_{A-G}(o, o)=1 \text {. }
$$

If $o$ is a non singular point of $\operatorname{Simp}^{s . s}(A-G)$, then the tangent space $\operatorname{Ext}_{A-G}^{1}(o, o)$ of $o$ must be of dimension $n=\operatorname{dim}_{k} U$. This proves the only if condition above. The if part, is a little more involved. 
Remark 6.6 If the Lie algebra $\mathfrak{g}$ (or the Lie group $G$ ) is not reductive,

$$
\operatorname{Ext}_{A-\mathfrak{g}}^{1}\left(V_{i}, V_{j}\right)=H^{0}\left(\mathfrak{g}, \operatorname{Ext}_{A}^{1}\left(V_{i}, V_{j}\right)\right) \oplus H^{1}\left(\mathfrak{g}, \operatorname{Hom}_{A}\left(V_{i}, V_{j}\right)\right)
$$

where the component $H^{1}\left(\mathfrak{g}, \operatorname{Hom}_{A}\left(V_{i}, V_{j}\right)\right)$ may be non-zero. This component of the tangent space of the deformation functor measures the number of different $\mathfrak{g}$-structures on the same deformation of the family of $A$ modules $\left\{V_{i}\right\}_{i}$. If we are not interested in the specific $\mathfrak{g}$-structures, but only in isomorphism classes as $A$-modules, then we may suppress this component, by taking an appropriate quotient of the resulting $H$, and of $O\left(\operatorname{Simp}\left(A_{\mathfrak{g}}, \pi\right)\right.$, and we obtain a $k$-algebra

$$
O^{\mathrm{red}}(\operatorname{Simp}(A-\mathfrak{g}), \pi)
$$

which is simpler, therefore contains less information, but for which the set of simple modules is in one-to-one correspondence with the orbits of $\mathfrak{g}$. See $\S 8$ for examples.

\section{Tensor products and quantum groups}

Let $\underline{c}$ be a subcategory of $A$-mod, with a trivializing functor $\pi$. Suppose given a tensor product on the category $\underline{c}$, i.e. a bi-functor

$$
\otimes: \underline{c} \times \underline{c} \longrightarrow \underline{c}
$$

which is a faithful imbedding, consistent with $\pi$, with some extra structure. In particular there should exist natural isomorphisms,

$$
\alpha_{-,-,-}:((-\otimes-) \otimes-) \simeq(-\otimes(-\otimes-))
$$

satisfying the Mac Lane pentagon condition,

$$
\begin{gathered}
i d_{X} \otimes \alpha_{Y, Z, W} \circ \alpha_{X, Y \otimes Z, W} \circ \alpha_{X, Y, Z} \otimes i d_{W} \\
=\alpha_{X, Y, Z \otimes W} \circ \alpha_{X \otimes Y, Z, W} .
\end{gathered}
$$

Consider the exact functor,

$$
\Delta: \underline{c} \rightarrow \underline{c} \times \underline{c},
$$

defined by $\Delta(V)=V \times V$. Then, since all of these functors are imbeddings, there exist homomorphisms of $k$-algebras,

$$
\mathcal{O}(\underline{c}, \pi) \rightarrow \mathcal{O}(\underline{c} \otimes \underline{c}, \pi) \simeq \mathcal{O}(\underline{c} \times \underline{c}, \pi) \rightarrow \mathcal{O}(\underline{c}, \pi)
$$


such that,

$$
\mathcal{O}(\underline{c} \times \underline{c}, \pi) \simeq \mathcal{O}(\underline{c}, \pi) \otimes \mathcal{O}(\underline{c}, \pi),
$$

and such that the last morphism of (1) is the multiplication morphism of the $k$-algebra $\mathcal{O}(\underline{c}, \pi)$.

The Mac Lane pentagon condition guaranties that the first morphism of (1) becomes an associative co-algebra structure on $\mathcal{O}(\underline{c}, \pi)$. Clearly any extra functorial symmetry one may want to consider on $\underline{c}$, will show up in the corresponding $k$-algebra $\mathcal{O}(\underline{c}, \pi)$.

\section{Examples}

\section{The noncommutative projective line}

Let $A=k\left[x_{0}, x_{1}\right]$, and consider the usual $k^{*}$-action. We shall compute the space $\operatorname{Spec}(A) / k^{*}$. The subcategory $\operatorname{Simp}\left(A-k^{*}\right)$ of $A-k^{*}$-modules consists of the origin $V_{3}$, the lines through the origin $V_{2}(l)$, and the generic point $V_{1}=A$. The trivializing functor (see $\S 3$ ),

$$
\pi: A-k^{*}-\bmod \longrightarrow k-\bmod
$$

has the values,

$$
\pi\left(V_{1}\right)=k, \pi\left(V_{2}(l)\right)=k, \pi\left(V_{3}\right)=k .
$$

The noncommutative orbit space is given by the hull of the deformation functor, i.e. by $\left(H_{i, j}\right)$. Since $H^{p}\left(k^{*},-\right)=0$ for $\mathrm{p} \geq 1$, we may use the Proposition (5.2), and we obtain,

$$
\operatorname{Ext}_{A-k^{*}}\left(V_{i}, V_{j}\right)=\operatorname{Ext}_{A}\left(V_{i}, V_{j}\right)^{k^{*}}
$$

It is easy to compute the different ext-groups, we find:

$$
\begin{aligned}
& \operatorname{Ext}_{A}^{1}\left(V_{i}, V_{j}\right)=0, \quad \text { for } i=1, j=1,2,3 . \\
& \operatorname{Ext}_{A}^{1}\left(V_{2}(l), V_{1}\right)=V_{2}(l)=A /\left(\alpha x_{0}+\beta x_{1}\right) \\
& \operatorname{Ext}_{A}^{1}\left(V_{2}(l), V_{2}(l)\right)=V_{2}(l) \\
& \operatorname{Ext}_{A}^{1}\left(V_{2}(l), V_{2}\left(l^{\prime}\right)\right)=0 \quad \text { if } l \neq l^{\prime} \\
& \operatorname{Ext}_{A}^{1}\left(V_{2}(l), V_{3}\right)=V_{3}=k \\
& \operatorname{Ext}_{A}^{1}\left(V_{3}, V_{1}\right)=0 \\
& \operatorname{Ext}_{A}^{1}\left(V_{3}, V_{2}(l)\right)=V_{3}=k \\
& \operatorname{Ext}_{A}^{1}\left(V_{3}, V_{3}\right)=k^{2} .
\end{aligned}
$$


Using the results of $\S 5$, we obtain for the invariants

$$
\begin{aligned}
& \operatorname{Ext}_{A}^{1}\left(V_{i}, V_{j}\right)^{k^{*}}=0, \quad \text { for } i=1, j=1,2,3 . \\
& \operatorname{Ext}_{A}^{1}\left(V_{2}(l), V_{1}\right)^{k^{*}}=k \text { represented by } \xi=l \\
& \operatorname{Ext}_{A}^{1}\left(V_{2}(l), V_{2}(l)\right)^{k^{*}}=k \text { represented by } \xi=l \\
& \operatorname{Ext}_{A}^{1}\left(V_{2}(l), V_{2}\left(l^{\prime}\right)\right)^{k^{*}}=0 \text { if } l \neq l^{\prime} \\
& \operatorname{Ext}_{A}^{1}\left(V_{2}(l), V_{3}\right)^{k^{*}}=0 \\
& \operatorname{Ext}_{A}^{1}\left(V_{3}, V_{1}\right)^{k^{*}}=0 \\
& \operatorname{Ext}_{A}^{1}\left(V_{3}, V_{2}(l)\right)^{k^{*}}=0 \\
& \operatorname{Ext}_{A}^{1}\left(V_{3}, V_{3}\right)^{k^{*}}=0 .
\end{aligned}
$$

The corresponding quotient becomes the infinite matrix algebra of the form,

$$
\operatorname{Spec}(A) / k^{*}:=O\left(\operatorname{Simp}\left(A-k^{*}\right), \pi\right)=\left(\begin{array}{ccc}
k & 0 & 0 \\
k\left[t_{2}(l)\right] t_{2,1} & k\left[t_{2}(l)\right] & 0 \\
0 & 0 & k
\end{array}\right)
$$

where $l$ runs through all the points in the ordinary projective line. We observe that the special point, corresponding to the isolated orbit, i.e. the origin, stays isolated, even infinitesimally. There are, however, adjacencies between the formal points corresponding to the lines through the origin, and the generic point corresponding to the generic point of the ordinary projective line.

Suppose that we localize, say in $x_{0}$, i.e. that we restrict to the

$$
\operatorname{Spec}\left(A_{\left\{x_{0}\right\}}-k^{*}\right)=\left\{V_{1}=A_{\left\{x_{0}\right\}}, V_{2}(l)=A_{\left\{x_{0}\right\}} /(l)\right\}
$$

then we find,

$$
\pi\left(V_{1}\right)=k\left[x_{1} / x_{0}\right], \pi\left(V_{2}(l)\right)=k,
$$

for all $l$. The exts in the new category looks like,

$$
\begin{aligned}
& \operatorname{Ext}_{A_{\left\{x_{0}\right\}}}^{1}\left(V_{i}, V_{j}\right)^{k^{*}}=0, \quad \text { for } i=1, j=1,2 . \\
& \operatorname{Ext}_{A_{\left\{x_{0}\right\}}}^{1}\left(V_{2}(l), V_{1}\right)^{k^{*}}=k \text { represented by } \xi=l \\
& \operatorname{Ext}_{A_{\left\{x_{0}\right\}}}^{1}\left(V_{2}(l), V_{2}(l)\right)^{k^{*}}=k \text { represented by } \xi=l \\
& \operatorname{Ext}_{A_{\left\{x_{0}\right\}}}^{1}\left(V_{2}(l), V_{2}\left(l^{\prime}\right)\right)^{k^{*}}=0 \text { if } l \neq l^{\prime}
\end{aligned}
$$

With this we find that $\operatorname{Spec}\left(A_{\left\{x_{0}\right\}}\right) / k^{*}$ is the subalgebra,

$$
\mathcal{O}\left(\operatorname{Simp}^{*}\left(A_{\left\{x_{0}\right\}}-k^{*}\right), \pi\right)=\left\{\left(\begin{array}{cc}
f\left(x_{1} / x_{0}\right) & 0 \\
\psi\left(f\left(x_{1} / x_{0}\right)\right) t_{2,1} & \left.f\left(x_{1} / x_{0}\right)\right]
\end{array}\right)\right\},
$$

of the algebra,

$$
\left(\begin{array}{cc}
\operatorname{End}_{k}\left(k\left[x_{1} / x_{0}\right]\right) & 0 \\
k\left[\left[x_{1} / x_{0}\right]\right] t_{2,1} & k\left[\left[t_{2}(l)\right]\right]
\end{array}\right)
$$


where $\psi$ is some derivation of $\operatorname{Der}_{k}\left(k\left[x_{1} / x_{0}\right]\right)$ and $f$ runs through $k\left[x_{1} / x_{0}\right]$, as expected, see the Theorem (3.2).

It is therefore clear that the noncommutative version of the projective line contains the geometric projective line.

If we consider, instead of the action by the group $k^{*}$, the action of the Lie algebra $\mathfrak{g}$ generated by the Euler vectorfield $\delta_{0}=x_{0} \partial / \partial x_{0}+x_{1} \partial / \partial x_{1}$, we get a different picture stemming from the fact that $\mathfrak{g}$ has cohomology. The subcategory $\operatorname{Spec}(A-\mathfrak{g})$ of $A-\mathfrak{g}$-modules consists of the origin $V_{3}$, the lines through the origin $V_{2}(l)$, and the generic point $V_{1}$. The trivializing functor

$$
\pi: A-\mathfrak{g}-\bmod \longrightarrow k-\bmod
$$

has the values,

$$
\pi\left(V_{1}\right)=k, \pi\left(V_{2}(l)\right)=k, \pi\left(V_{3}\right)=k
$$

Since there are no $\pi$-incidences, the noncommutative orbit space $\operatorname{Spec}(A) / \mathfrak{g}$ is given by the hull of the deformation functor, i.e. by $\left(H_{i, j}\right)$, as above. However, here we cannot use the result (6.2), since for most $\mathfrak{g}$-modules $\mathrm{V}$, $H^{1}(\mathfrak{g}, V)=V / \delta_{0} V \neq 0$. In fact we get,

$$
\begin{aligned}
& \operatorname{Ext}_{A-\mathfrak{g}}^{1}\left(V_{i}, V_{j}\right)=\operatorname{Ext}_{A}^{1}\left(V_{i}, V_{j}\right)^{\mathfrak{g}} \oplus H^{1}\left(\mathfrak{g}, \operatorname{Hom}_{A}\left(V_{i}, V_{j}\right)\right) \\
& \operatorname{Ext}_{A-\mathfrak{g}}^{2}\left(V_{i}, V_{j}\right)=H^{1}\left(\mathfrak{g}, \operatorname{Ext}_{A}^{1}\left(V_{i}, V_{j}\right)\right)
\end{aligned}
$$

This implies that

$$
\begin{aligned}
\operatorname{Ext}_{A-\mathfrak{g}}^{1}\left(V_{1}, V_{j}\right) & =H^{1}\left(\mathfrak{g}, \operatorname{Hom}_{A}\left(V_{1}, V_{j}\right)\right)=k \text { for } j=1,2,3 . \\
\operatorname{Ext}_{A-\mathfrak{g}}^{1}\left(V_{2}(l), V_{j}\right) & =\operatorname{Ext}_{A}^{1}\left(V_{2}, V_{j}\right)^{\mathfrak{g}} \oplus H^{1}\left(\mathfrak{g}, \operatorname{Hom}_{A}\left(V_{2}, V_{j}\right)\right) \\
& =k \oplus 0 \quad \text { for } j=1 \\
& =k \oplus k \quad \text { for } V_{j}=V_{2}(l) \\
& =0 \oplus 0 \quad \text { for } V_{j}=V_{2}\left(l^{\prime}\right) l \neq l^{\prime} \\
& =0 \oplus k \quad \text { for } j=3 \\
\operatorname{Ext}_{A-\mathfrak{g}}^{1}\left(V_{3}, V_{j}\right) & =\operatorname{Ext}_{A}^{1}\left(V_{3}, V_{j}\right)^{\mathfrak{g}} \oplus H^{1}\left(\mathfrak{g}, \operatorname{Hom}_{A}\left(V_{3}, V_{j}\right)\right) \\
& =0 \oplus 0 \quad \text { for } j=1 \\
& =0 \oplus 0 \quad \text { for } j=2 \\
& =0 \oplus k \quad \text { for } j=3 \\
\operatorname{Ext}_{A-\mathfrak{g}}^{2}\left(V_{i}, V_{j}\right) & =H^{1}\left(\mathfrak{g}, \operatorname{Ext}_{A}^{1}\left(V_{i}, V_{j}\right)\right) \\
& =0 \quad \text { for } i=1, j=1,2,3 \\
& =k \quad \text { for } i=2, j=1 \\
& =k \quad \text { for } V_{i}=V_{2}(l), V_{j}=V_{2}(l) \\
& =0 \quad \text { for } V_{i}=V_{2}(l), V_{j}=V_{2}\left(l^{\prime}\right), l \neq l^{\prime} \\
& =k \quad \text { for } i=3, j=3 .
\end{aligned}
$$


It follows that $\operatorname{Spec}(A) / \mathfrak{g}$ is given by the rather complicated looking $k$-algebra generated by

$$
\left(\begin{array}{ccc}
k\left[\left[t_{1}\right]\right] & t_{1,2}(l) & t_{1,3}(l) \\
u_{2,1}(l) & k\left[\left[t_{2}(l), u_{2}(l)\right]\right] & t_{2,3}(l) \\
0 & 0 & k\left[\left[t_{3}\right]\right]
\end{array}\right)
$$

with some relations.

The moduli space of simple singularities, the $A_{2}$ case

We shall consider the Weierstrass family $F:=F\left(t_{0}, t_{1}, x, y\right)=x^{3}-y^{2}+$ $t_{1} x+t_{0}$, parametrized by the $k$-algebra $A:=k\left[t_{0}, t_{1}\right]$, and the corresponding Kodaira-Spencer kernel $\mathfrak{g} \subseteq \operatorname{Der}_{k}(A)$ see [9], generated by,

$$
\begin{aligned}
& \delta_{0}=3 t_{0} \frac{\partial}{\partial t_{0}}+2 t_{1} \frac{\partial}{\partial t_{1}} \\
& \delta_{1}=2 t_{1}^{2} \frac{\partial}{\partial t_{0}}-9 t_{0} \frac{\partial}{\partial t_{1}}
\end{aligned}
$$

We claim that the moduli space consisting of the three singularities in the family $F$, is given as the quotient space $\operatorname{Spec}(A) / \mathfrak{g}$. We must therefore consider the diagram $\operatorname{Simp}(A-\mathfrak{g})$, consisting of the three $A-\mathfrak{g}$-modules, $V_{1}=k\left[t_{0}, t_{1}\right], V_{2}=k\left[t_{0}, t_{1}\right] /(\Delta)$, where $\Delta=27 t_{0}^{2}+4 t_{1}^{3}$ is the discriminant of $F$, and finally $V_{3}=k$ corresponding to the origin.

As above we find that

$$
\pi=H^{0}(\mathfrak{g},-): A-\mathfrak{g}-\bmod \longrightarrow k-\bmod
$$

defines three points,

$$
\pi\left(V_{1}\right)=k, \pi\left(V_{2}\right)=k, \pi\left(V_{3}\right)=k
$$

with no incidences. Since it is easy to see that

$$
H^{2}\left(\mathfrak{g}, \operatorname{Hom}_{A}\left(V_{i}, V_{j}\right)\right)=0
$$

we find,

$$
\operatorname{Ext}_{A-\mathfrak{g}}^{1}\left(V_{i}, V_{j}\right)=H^{0}\left(\mathfrak{g}, \operatorname{Ext}_{A}^{1}\left(V_{i}, V_{j}\right)\right) \oplus H^{1}\left(\mathfrak{g}, \operatorname{Hom}_{A}\left(V_{i}, V_{j}\right)\right)
$$


which implies,

$$
\begin{aligned}
\operatorname{Ext}_{A-\mathfrak{g}}^{1}\left(V_{1}, V_{j}\right) & =H^{1}\left(\mathfrak{g}, \operatorname{Hom}_{A}\left(V_{1}, V_{j}\right)\right)=k \text { for } j=1,2,3 \\
\operatorname{Ext}_{A-\mathfrak{g}}^{1}\left(V_{2}, V_{j}\right) & =H^{0}\left(\mathfrak{g}, \operatorname{Ext}_{A}^{1}\left(V_{2}, V_{j}\right)\right) \oplus H^{1}\left(\mathfrak{g}, \operatorname{Hom}_{A}\left(V_{2}, V_{j}\right)\right) \\
& =0 \oplus 0 \quad \text { for } j=1 \\
& =0 \oplus k \quad \text { for } j=2 \\
& =0 \oplus k \text { for } j=3 \\
\operatorname{Ext}_{A-\mathfrak{g}}^{1}\left(V_{3}, V_{j}\right) & =\operatorname{Ext}_{A}^{1}\left(V_{3}, V_{j}\right)^{\mathfrak{g}} \oplus H^{1}\left(\mathfrak{g}, \operatorname{Hom}_{A}\left(V_{3}, V_{j}\right)\right) \\
& =0 \oplus 0 \quad \text { for } j=1 \\
& =0 \oplus 0 \text { for } j=2 \\
& =0 \oplus k \text { for } j=3 .
\end{aligned}
$$

Moreover, for all $i, j=1,2,3$,

$$
\operatorname{Ext}_{A-\mathfrak{g}}^{2}\left(V_{i}, V_{j}\right) \subseteq H^{0}\left(\mathfrak{g}, \operatorname{Ext}_{A}^{2}\left(V_{i}, V_{j}\right)\right) \oplus H^{1}\left(\mathfrak{g}, \operatorname{Ext}_{A}^{1}\left(V_{i}, V_{j}\right)\right)=0 .
$$

The moduli space is therefore given by the $k$-algebra (freely generated by),

$$
O(\operatorname{Simp}(A-\mathfrak{g}), \pi)=\left(\begin{array}{ccc}
k\left[\left[t_{1,1}\right]\right] & t_{1,2} & t_{1,3} \\
0 & k\left[\left[t_{2,2}\right]\right] & t_{2,3} \\
0 & 0 & k\left[\left[t_{3,3}\right]\right]
\end{array}\right)
$$

which has a reduced quotient, given by the matrices of the form,

$$
\left(\begin{array}{ccc}
k & k t_{1,2} & k t_{1,3} \oplus k t_{1,2} t_{2,3} \\
0 & k & k t_{2,3} \\
0 & 0 & k
\end{array}\right)
$$

which is the $k$-algebra of the non-commuting adjacency diagram corresponding to the Weierstrass family, see [9],

$$
\begin{aligned}
& t_{2,3}: \text { cusp } \rightarrow \text { node } \\
& t_{1,2}: \text { node } \rightarrow \text { ellipt } \\
& t_{1,2} t_{2,3}: \text { cusp } \longrightarrow \text { ellipt } \\
& t_{1,3}: \text { cusp } \rightarrow \text { ellipt }
\end{aligned}
$$

Notice that $\mathfrak{g}$ is a rank $2 A$-module, such that we may expect to find exact sequences of $A-\mathfrak{g}$-modules,

$$
\begin{aligned}
& 0 \longrightarrow A \longrightarrow \mathfrak{g} \longrightarrow A \longrightarrow 0 \\
& 0 \longrightarrow A /(\Delta) \longrightarrow \mathfrak{g} \otimes_{A} A /(\Delta) \longrightarrow A /(\Delta) \longrightarrow 0 \\
& 0 \longrightarrow A /\left(t_{0}, t_{1}\right) \longrightarrow \mathfrak{g} \otimes_{A} k(o) \longrightarrow A /\left(t_{0}, t_{1}\right) \longrightarrow 0
\end{aligned}
$$


explaining the diagonal tangent structure of the moduli space,

$$
\left(\begin{array}{ccc}
k\left[\left[t_{1,1}\right]\right] & t_{1,2} & t_{1,3} \\
0 & k\left[\left[t_{2,2}\right]\right] & t_{2,3} \\
0 & 0 & k\left[\left[t_{3,3}\right]\right]
\end{array}\right) .
$$

The reduced moduli is, however, given by,

$$
O^{\mathrm{red}}(\operatorname{Simp}(A-\mathfrak{g}), \pi)=\left(\begin{array}{ccc}
k & 0 & 0 \\
0 & k & 0 \\
0 & 0 & k
\end{array}\right)
$$

i.e. the obvious 3-point space.

\section{The moduli of endomorphisms. The dimension 2 case}

We shall compute the (noncommutative) space of invariants

$$
\operatorname{End}_{k}\left(k^{n}\right) / \mathrm{GL}_{n}(k)
$$

for $k=\mathbb{C}$ and $n=2$. (For $n \geq 3$ see a forthcoming paper by Arvid Siqveland [19]). Refering to $\S 4$, it suffices to compute the noncommutative formal moduli for the longest chain of infinitesimal incidences, i.e. the family of formal Jordan forms. Let

$$
A=O_{\operatorname{End}_{k}\left(k^{2}\right)}\left(\operatorname{End}_{k}\left(k^{2}\right)\right)
$$

then

$$
A=k\left[x_{1,1}, x_{1,2}, x_{2,1}, x_{2,2}\right]
$$

The group $G:=\mathrm{GL}_{2}(k)$ acts on $\operatorname{End}_{k}\left(k^{2}\right)$ by conjugation, and there are two Jordan forms of interest,

$$
\left(\begin{array}{ll}
\lambda & 0 \\
0 & \lambda
\end{array}\right)
$$

and

$$
\left(\begin{array}{ll}
\lambda & 1 \\
0 & \lambda
\end{array}\right)
$$

corresponding to orbits $V_{2}$ of dimension 0 and $V_{1}$ of dimension 2, respectively, in $\operatorname{End}_{k}\left(k^{2}\right)$. According to $\S 3$, since

$$
H^{0}\left(G, V_{i}\right)=k, i=1,2
$$

we should expect $\operatorname{End}_{k}\left(k^{2}\right) / \mathrm{GL}_{2}(k)$ to be an algebraization of the formal moduli of the family of $A-G$-modules $\left\{V_{i}\right\}_{i=1.2}$, i.e.

$$
H:=H\left(\left\{V_{i}\right\}_{i=1.2}\right)
$$


The orbits, as $A$-modules, are given by:

$$
\begin{aligned}
& V_{1}=k\left[x_{i . j}\right] /\left(s_{1}-2 \lambda, s_{2}-\lambda^{2}\right) \\
& V_{2}=k\left[x_{i . j}\right] /\left(x_{1,1}-\lambda, x_{1,2}, x_{2,1}, x_{2,2}-\lambda\right) .
\end{aligned}
$$

Now it is clear that we may assume $\lambda=0$. We find $A$-free resolutions,

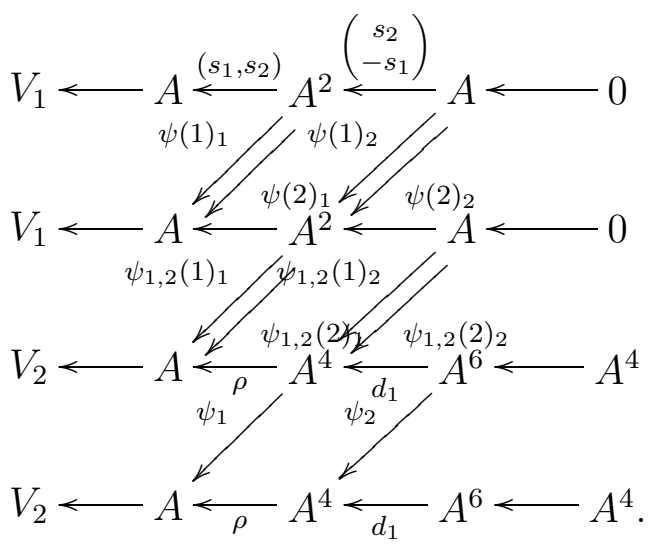

Here,

$$
\begin{array}{rlrl}
\psi(1)_{1} & =(1,0) & \psi(2)_{1}=(0,1) \\
\psi(1)_{2}=\left(\begin{array}{l}
0 \\
1
\end{array}\right) & \psi(2)_{2}=\left(\begin{array}{c}
-1 \\
0
\end{array}\right) \\
\psi_{(1,2)}(1)_{1}=(1,0) & \psi_{(1,2)}(2)_{1}=(0,1) \\
\psi_{(1,2)}(1)_{2}=\left(\begin{array}{c}
x_{2,2} \\
-x_{2,1} \\
0 \\
0
\end{array}\right) & \psi_{(1,2)}(2)_{2}=\left(\begin{array}{c}
-1 \\
0 \\
0 \\
-1
\end{array}\right)
\end{array}
$$

and

$$
\begin{aligned}
\rho & =\left(\begin{array}{llllll}
x_{1,1} & x_{1,2} & x_{2,1} & x_{2,2}
\end{array}\right) \\
d_{1} & =\left(\begin{array}{cccccc}
x_{1,2} & x_{2,1} & x_{2,2} & 0 & 0 & 0 \\
-x_{1,1} & 0 & 0 & x_{2,1} & x_{2,2} & 0 \\
0 & -x_{1,1} & 0 & -x_{1,2} & 0 & x_{2,2} \\
0 & 0 & -x_{1,1} & 0 & -x_{1,2} & -x_{2,1}
\end{array}\right) \\
\psi_{1} & =\left(\begin{array}{cccc}
1 & 0 & 0 & 1
\end{array}\right) \\
\psi_{2} & =\left(\begin{array}{cccccc}
0 & 0 & -1 & 0 & 0 & 0 \\
1 & 0 & 0 & 0 & -1 & 0 \\
0 & 1 & 0 & 0 & 0 & -1 \\
0 & 0 & 1 & 0 & 0 & 0
\end{array}\right)
\end{aligned}
$$


From this diagram we easily compute $\operatorname{Ext}_{A}^{1}\left(V_{i}, V_{j}\right)$, and since $G$ is reductive, also $\operatorname{Ext}_{A-G}^{1}\left(V_{i}, V_{j}\right)=\operatorname{Ext}_{A}^{1}\left(V_{i}, V_{j}\right)^{G}$. We obtain,

$$
\operatorname{Ext}_{A-G}^{1}\left(V_{i}, V_{j}\right)= \begin{cases}k^{2} & \text { for } i=1 \\ k & \text { for } i=j=2 \\ 0 & \text { for } i=2, j=1\end{cases}
$$

which means that the tangent space of $H$ is given by,

$$
\left(\begin{array}{cc}
k^{2} & k^{2} \\
0 & k^{1}
\end{array}\right) \text {. }
$$

Now,

$$
\begin{aligned}
& \operatorname{Ext}_{A-G}^{2}\left(V_{1}, V_{1}\right)=k \cdot \eta_{1,1} \\
& \operatorname{Ext}_{A-G}^{2}\left(V_{1}, V_{2}\right)=k \cdot \eta_{1,2}
\end{aligned}
$$

and we compute the cup products and the Massey products of the basis elements of the $\operatorname{Ext}_{A-G}^{1}\left(V_{i}, V_{j}\right)=\operatorname{Ext}_{A}^{1}\left(V_{i}, V_{j}\right)^{G}$,

$$
\begin{aligned}
& s_{1}^{*} \cup s_{2}^{*}=-s_{2}^{*} \cup s_{1}^{*}=\eta_{1,1}, s^{*} \cup s^{*}=0 \\
& t_{1}^{*} \cup s^{*}=0, t_{2}^{*} \cup s^{*}=-2 \cdot \eta_{1,2}, \\
& s_{1}^{*} \cup t_{1}^{*}=0, s_{1}^{*} \cup t_{2}^{*}=\eta_{1,2}, s_{2}^{*} \cup t_{1}^{*}=-\eta_{1,2}, s_{2}^{*} \cup t_{2}^{*}=0 \\
& <t_{1}^{*}, s^{*}, s^{*}>=\eta_{1,2}, \quad<s_{1}^{*}, t_{1}^{*}, s^{*}>=0 .
\end{aligned}
$$

This proves that in $H$ there are relations of the form,

$$
s_{1} s_{2}=s_{2} s_{1}, \quad t_{1} s^{2}-2 \cdot t_{2} s+s_{1} t_{2}-s_{2} t_{1}=0
$$

It follows that,

$$
H\left(\left\{V_{i}\right\}\right)=\left(\begin{array}{cc}
k\left[\left[s_{1}, s_{2}\right]\right] & <t_{1}, t_{2}> \\
0 & k[[s]]
\end{array}\right)
$$

subject to the relation $t_{1} s^{2}-s_{2} t_{1}-2 \cdot t_{2} s+s_{1} t_{2}=0$. From this it is clear that,

$$
\operatorname{End}_{k}\left(k^{2}\right) / \mathrm{GL}_{2}(k)=\tilde{H}\left(\left\{V_{i}\right\}\right)=\left(\begin{array}{cc}
k\left[s_{1}, s_{2}\right] & <t_{1}, t_{2}> \\
0 & k[s]
\end{array}\right)
$$

subject to the relation $t_{1} s^{2}-s_{2} t_{1}-2 \cdot t_{2} s+s_{1} t_{2}=0$ in the upper right corner, $H_{1,2}=k\left[s_{1}, s_{2}\right]<t_{1}, t_{2}>k[s]$.

The $k$-points of $\operatorname{End}_{k}\left(k^{2}\right) / \mathrm{GL}_{2}(k)$ are therefore stratified into two strata, a plane parametrized by $\left(s_{1}, s_{2}\right)$, corresponding to the semi-simple orbits, and to the (maximal dimensional) orbits given by the Jordan form,

$$
\left(\begin{array}{ll}
\lambda & 1 \\
0 & \lambda
\end{array}\right)
$$


and a line parametrized by $(s)$, corresponding to the orbits of dimension 0 , i.e. to the Jordan forms,

$$
\left(\begin{array}{ll}
s & 0 \\
0 & s
\end{array}\right)
$$

There are two tangents from a point $\left(s_{1}, s_{2}\right)$ in the plane to the point $s$ on the line if and only if he relation above is trivial, i.e. iff $s_{1}=2 s$ and $s_{2}=s^{2}=0$.

The versal family, $\tilde{\mathcal{V}}$, i.e. the action of $A$ on $\tilde{\mathcal{V}}=H \otimes \mathcal{V}$. This is easily done by using the $k$-linear and GL(2)-invariant section of the morphism $A \rightarrow V_{1}=A /\left(s_{1}, s_{2}\right)$, see $\S 5$, induced by fixing a $k$-basis,

$$
\left\{x_{1,1}^{n_{0}} x_{1,2}^{n_{1}} x_{2,1}^{n_{2}}=: x_{1,1}^{n_{0}} v_{0}\right\}_{0 \leq n_{0} \leq 1,0 \leq n_{1}, n_{2}}
$$

for $V_{1}$, mapping, multiplicatively, $x_{1,1}$ to $1 / 2\left(x_{1,1}-x_{2,2}\right)$, and $x_{i, j}, i \neq j$ to $x_{i, j}$. We obtain,

$$
\tilde{\mathcal{V}}=\left(H\left(\left\{V_{i}\right\}\right)_{i, j} \otimes V_{j}\right)=\left(\begin{array}{cc}
k\left[s_{1}, s_{2}\right] \otimes V_{1} & <t_{1}, t_{2}>\otimes V_{2} \\
0 & k[s] \otimes V_{2}
\end{array}\right)
$$

where $V_{2}=k$, subject to the relation in $H_{1,2}=k\left[s_{1}, s_{2}\right]<t_{1}, t_{2}>k[s]$,

$$
t_{1} s^{2}-s_{2} t_{1}-2 \cdot t_{2} s+s_{1} t_{2}=0
$$

with the $k\left[x_{i, j}\right]$-action given by,

$$
\left(\begin{array}{cc}
1 \otimes v_{1} & 0 \\
0 & 1 \otimes v_{2}
\end{array}\right) x_{i, j}=\left(\begin{array}{cc}
1 \otimes v_{1} x_{i, j} & 0 \\
0 & 0
\end{array}\right)
$$

if $i \neq j$, and,

$$
\begin{aligned}
& \left(\begin{array}{cc}
1 \otimes v_{0} & 0 \\
0 & 1 \otimes v_{2}
\end{array}\right) x_{1,1}=\left(\begin{array}{cc}
1 \otimes v_{0} x_{1,1}-1 / 2 s_{1} \otimes v_{0} & -1 / 2 t_{1} \otimes v_{0}^{\prime} \\
0 & -s \otimes v_{2}
\end{array}\right) \\
& \left(\begin{array}{cc}
1 \otimes v_{0} & 0 \\
0 & 1 \otimes v_{2}
\end{array}\right) x_{2,2}=\left(\begin{array}{cc}
-1 \otimes v_{0} x_{1,1}-1 / 2 s_{1} \otimes v_{0} & -1 / 2 t_{1} \otimes v_{0}^{\prime} \\
0 & -s \otimes v_{2}
\end{array}\right)
\end{aligned}
$$

Moreover, the first row of the matrix multiplied with $x_{i, i}$, for $v_{1}=v_{0} x_{1,1}$, looks like, for $i=1$;

$$
\begin{aligned}
& -1 \otimes v_{0} x_{1,2} x_{2,1}-1 / 2 s_{1} \otimes v_{0} x_{1,1}+s_{2} \otimes v_{0}+t_{2} \otimes v_{0}^{\prime}-1 / 2 t_{1} s \otimes v_{0}^{\prime} \\
& -(s / 2)^{2} /(1-(s / 2)) t_{1} \otimes v_{0}^{\prime},
\end{aligned}
$$

and for $i=2$,

$$
\begin{aligned}
& 1 \otimes v_{0} x_{1,2} x_{2,1}-1 / 2 s_{1} \otimes v_{0} x_{1,1}-s_{2} \otimes v_{0}-t_{2} \otimes v_{0}^{\prime}+1 / 2 t_{1} s \otimes v_{0}^{\prime} \\
& \quad+(s / 2)^{2} /(1-(s / 2)) t_{1} \otimes v_{0}^{\prime},
\end{aligned}
$$

where $v_{0}^{\prime}$ is the image in $V_{2}$ of $v_{0} \in V_{1}$. 


\section{An example of Tord Romstad}

Let $A=k[x, y]$ and let the $A$-module $V=A /(x-2 y)$. Let the group $G=\mathbb{Z} /(2)$ be generated by $\tau$, and let $G$ act on $A$ as,

$$
\tau(x)=x, \tau(y)=x-y .
$$

When $\operatorname{char}(k) \neq 2$, it is easy to show that,

$$
\operatorname{Ext}_{A(G)}^{1}(V, V)=k, \operatorname{Ext}_{A(G)}^{2}(V, V)=0 .
$$

When $\operatorname{char}(k)=2$, a more complicate calculation shows that,

$$
\operatorname{Ext}_{A(G)}^{1}(V, V)=k^{2}, \operatorname{Ext}_{A(G)}^{2}(V, V)=k^{2} .
$$

Computing the cup and third-order Massey products one shows that,

$$
H^{A(G)}(V)=k\left\{\left\{t_{1}, t_{2}\right\}\right\} /\left(t_{2}^{2}, t_{1}^{2} t_{2}+t_{2} t_{1}^{2}\right),
$$

which should be compared with the introduction to $\S 6$, and to (6.3), see also [15].

\section{References}

[1] Artin, M.: On Azumaya Algebras and Finite Dimensional Representations of Rings. J. Algebra 11 (1969), 532-563.

[2] Artin, M., Tate, J. And Van Den Bergh, M.: Some algebras associated to automorphisms of elliptic curves. In The Grothendieck Festschrift, Vol 1, 33-85. Progr. Math. 86, Birkhäuser, Boston, 1990.

[3] Connes, A.: Géometrie Noncommutative. InterEditions, 1990.

[4] Formanek, E.: The polynomial identities and invariants of $n \times n$ matrices. CBMS Regional Conference Series in Mathematics 78. Published for the Conference Board of the Mathematical Sciences, Washington, DC; by the American Mathematical Society, Providence, RI, 1991.

[5] ILE, R.: Noncommutative deformations. $a b \neq b a$. Master Thesis, University of Oslo, 1990.

[6] Laudal, O. A.: Sur les limites projectives et inductives. Ann. Sci. École Norm. Sup. (3) 82 (1965), 241-296.

[7] Laudal, O. A.: Formal moduli of algebraic structures. Lecture Notes in Math. 754, Springer Verlag, 1979.

[8] Laudal, O. A.: Matric Massey products and formal moduli. In Algebra, Algebraic Topology and their interactions (Roos, J. E., ed.), 218-240. Lecture Notes in Math. 1183, Springer Verlag, 1986. 
[9] Laudal, O. A. and Pfister, G.: Local moduli and singularities. Lecture Notes in Math. 1310, Springer Verlag, 1988.

[10] Laudal, O.A.: Noncommutative deformations of modules. The Roos Festschrift volume (2). Homology Homotopy Appl. 4 (2002), no. 2, part 2, 357-396.

[11] Laudal, O. A.: A generalized Burnside theorem. Preprint Series vol. 42, Institute of Mathematics, University of Oslo, 1995.

[12] Laudal, O.A.: Noncommutative Algebraic Geometry. Max-PlanckInstitut für Mathematik, Preprint Series 2000 (115).

[13] Manin, Y. I.: Topics in Noncommutative Geometry. Princeton University Press, 1991.

[14] Procesi, C.: Non-commutative affine rings. Atti Accad. Naz. Lincei Mem. Cl. Sci. Fis. Mat. Natur. Sez. I (8) 8 (1967), 237-255.

[15] Romstad, T.: Non-commutative Algebraic Geometry Applied to Invariant Theory of Finite Group Actions. Cand. Scient. Thesis. Institute of Mathematics, University of Oslo, 2000.

[16] Rosenberg, A. L.: Noncommutative algebraic geometry and representations of quantized algebra. Mathematics and its applications 330, Klüwer, 1995.

[17] Schlessinger, M.: Functors of Artin rings. Trans. Amer. Math. Soc. 130 (1968), 208-222.

[18] Siqveland, A.: Matric Massey products and formal moduli. Thesis, Institute of Mathematics, University of Oslo, 1995.

[19] Siqveland, A.: The Noncommutative Moduli of Rank 3 Endomorphisms. Report Series No. 26, Buskerud College, Norway, 2001.

[20] Van Oystaeyen, F. and Verschoren A.: Non-commutative Algebraic Geometry. An Introduction. Lecture Notes in Math. 887, Springer-Verlag, 1981.

Recibido: 20 de febrero de 2002

Revisado: 30 de marzo de 2003

Olav Arnfinn Laudal

Matematisk Institutt

Universitetet i Oslo

Boks 1053, Blindern

NO 0316, Oslo

arnfinnl@math.uio.no 